MULTIRIDGELETS FOR TEXTURE ANALYSIS

\author{
by \\ Hong-Jun Yoon \\ BSEE, Soongsil University, 1995 \\ MSEE, Soongsil University, 1997 \\ MSEE, University of Wisconsin, Milwaukee, 1999
}

Submitted to the Graduate Faculty of

Swanson School of Engineering in partial fulfillment

of the requirements for the degree of

Doctor of Philosophy

University of Pittsburgh 


\section{UNIVERSITY OF PITTSBURGH \\ SWANSON SCHOOL OF ENGINEERING}

This dissertation was presented

by

Hong-Jun Yoon

It was defended on

July 28, 2011

and approved by

John Boston, PhD, Professor, Electrical and Computer Engineering Department

Luis F. Chaparro, PhD, Associate Professor, Electrical and Computer Engineering Department

Amro El-Jaroudi, PhD, Associate Professor, Electrical and Computer Engineering Department Mingui Sun, PhD, Professor, Neurological Surgery Department

Dissertation Director: Ching-Chung Li, PhD, Professor, Electrical and Computer Engineering

Department 
Copyright (C) by Hong-Jun Yoon

2011 


\title{
MULTIRIDGELETS FOR TEXTURE ANALYSIS
}

\author{
Hong-Jun Yoon, $\mathrm{PhD}$ \\ University of Pittsburgh, 2011
}

Directional wavelets have orientation selectivity and thus are able to efficiently represent highly anisotropic elements such as line segments and edges. Ridgelet transform is a kind of directional multi-resolution transform and has been successful in many image processing and texture analysis applications. The objective of this research is to develop multi-ridgelet transform by applying multiwavelet transform to the Radon transform so as to attain attractive improvements. By adapting the cardinal orthogonal multiwavelets to the ridgelet transform, it is shown that the proposed cardinal multiridgelet transform (CMRT) possesses cardinality, approximate translation invariance, and approximate rotation invariance simultaneously, whereas no single ridgelet transform can hold all these properties at the same time. These properties are beneficial to image texture analysis. This is demonstrated in three studies of texture analysis applications. Firstly a texture database retrieval study taking a portion of the Brodatz texture album as an example has demonstrated that the CMRT-based texture representation for database retrieval performed better than other directional wavelet methods. Secondly the study of the LCD mura defect detection was based upon the classification of simulated abnormalities with a linear support vector machine classifier, the CMRT-based analysis of defects were shown to provide efficient features for superior detection performance than other competitive methods. Lastly and the most importantly, a study on the prostate cancer tissue image classification was conducted. With the CMRT-based texture extraction, Gaussian kernel support vector machines have been 
developed to discriminate prostate cancer Gleason grade 3 versus grade 4. Based on a limited database of prostate specimens, one classifier was trained to have remarkable test performance. This approach is unquestionably promising and is worthy to be fully developed. 


\section{TABLE OF CONTENTS}

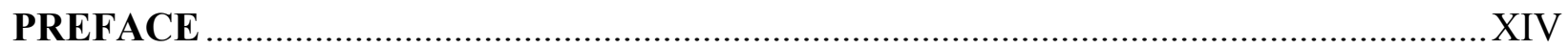

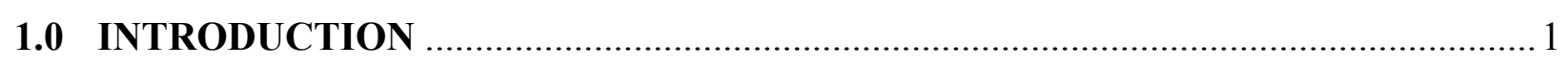

2.0 CARDINAL MULTIRIDGELET TRANSFORM ................................................. 4

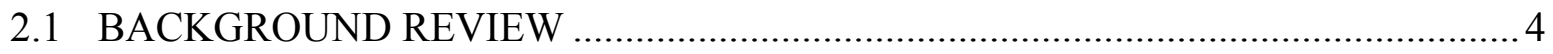

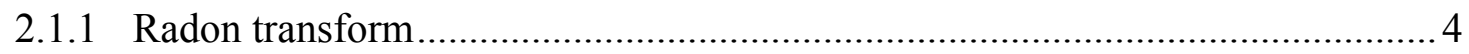

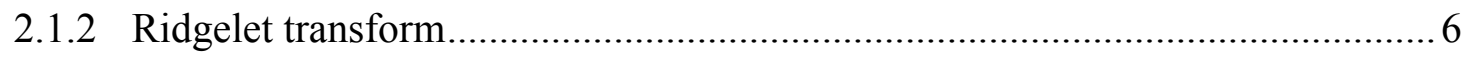

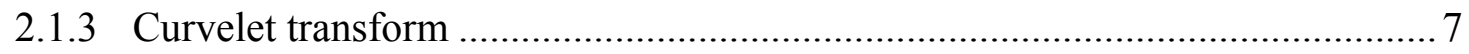

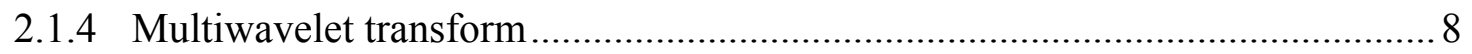

2.1.5 Cardinal multiwavelets........................................................................ 10

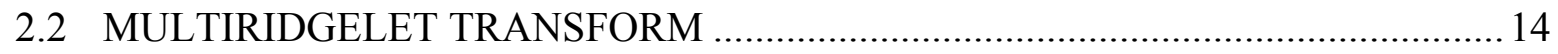

2.2.1 Continuous multiridgelet transform ......................................................... 14

2.2.2 Multiresolution analysis of multiridgelet transform ……................................ 17

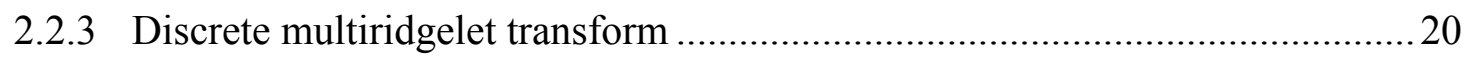

2.3 PROPERTIES OF CARDINAL MULTIRIDGELET TRANSFORM …….................23

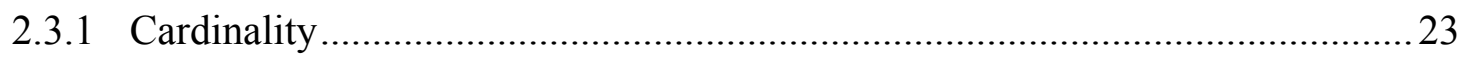

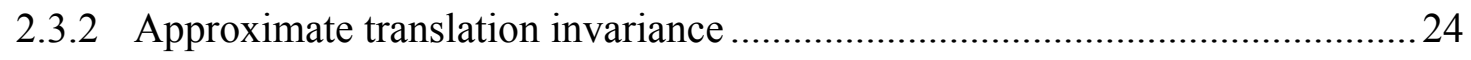

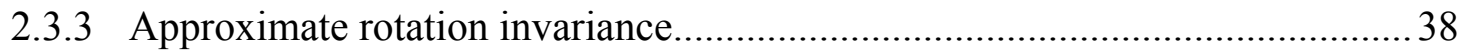

2.3.4 Advantages of CMRT-based texture analysis ................................................. 42 


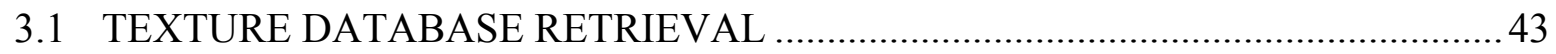

3.1.1 Feature vector from the cardinal multiridgelet transform ...............................4

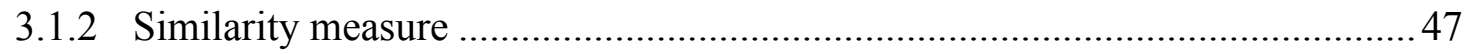

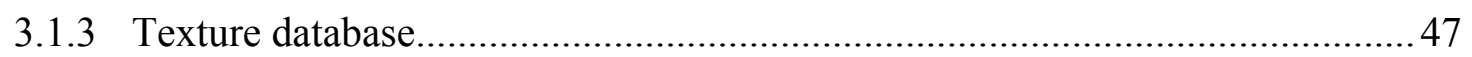

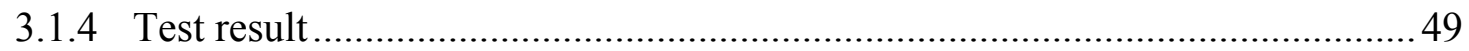

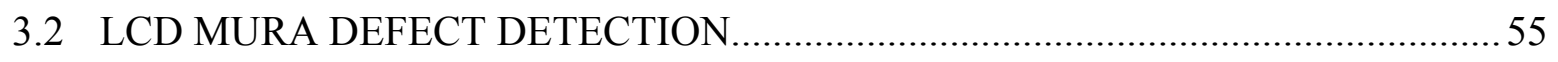

3.2.1 Linear support vector machine classifier .......................................................55

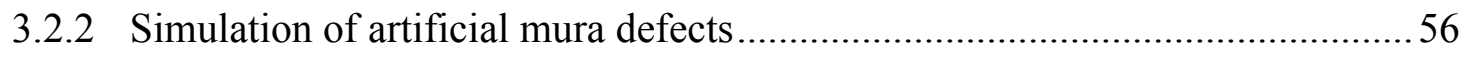

3.2.3 Feature extraction in CMRT domain .........................................................5

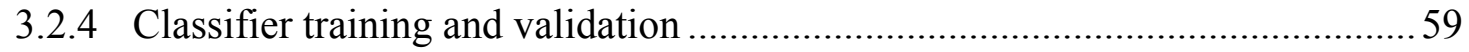

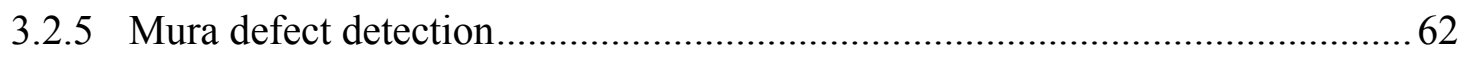

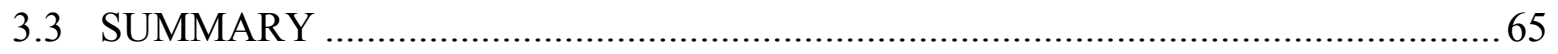

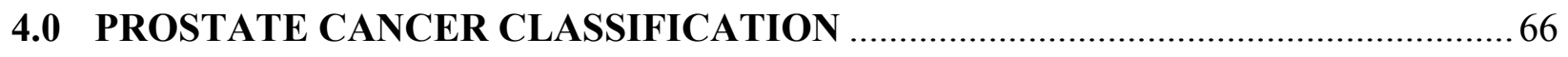

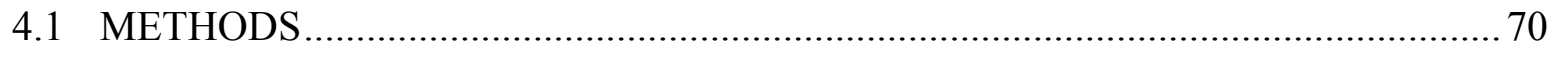

4.1.1 Prostate cancer image database .............................................................. 70

4.1.2 Feature extraction from cardinal multiridgelet packets .................................. 71

4.1.3 Non-linear support vector machine classifier ................................................73

4.1.4 Strategy in classifier design and cross-validation test.....................................76

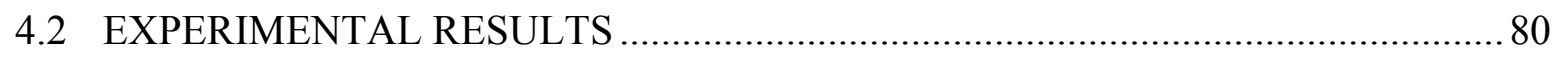

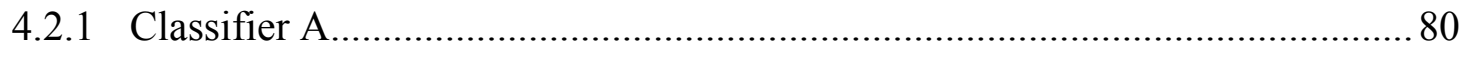

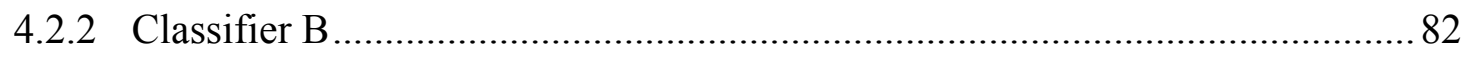

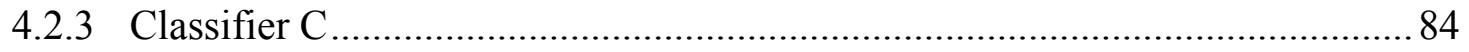




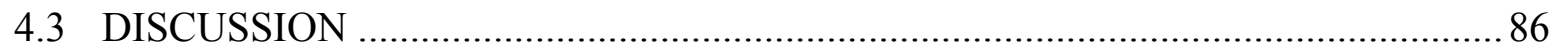

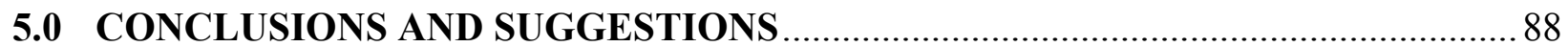

5.1 SUGGESTIONS FOR FURTHER RESEARCH............................................... 90

APPENDIX A

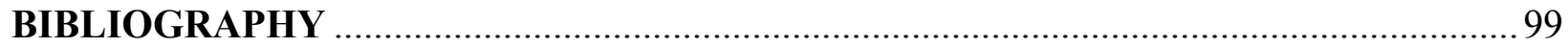




\section{LIST OF TABLES}

Table 1 Average retrieval rate for the 25 texture images in database at $\mathrm{C}=15$.

Table 2 Comparison of figures of merit in AUC of four methods, $95 \%$ confidence intervals in parenthesis.

Table 3 Mura defect detection accuracy of all four methods, AUC and $95 \%$ confidence intervals were determined by DBM MRMC software with PROPROC fit.

Table 4 Number of samples per each Gleason score in TMA-471 database. .71

Table 5. Training and testing results of RBF SVM Classifier A for prostate cancer Gleason grade 3 versus grade 4 , the result of the cardinal multiridgelet-based method listed in the first row and that of the curvelet-based method is in the second row. 81

Table 6. Training and testing results of RBF SVM Classifier B for prostate cancer Gleason grade 3 versus grade 4 .

Table 7 Training and testing results of RBF SVM Classifier C for prostate cancer Gleason grade 3 versus grade 4 .

Table 8 Parameters of the CMRT-based RBF SVM Classifier A for prostate cancer Gleason grade 3 versus grade 4 (16 $\mathrm{P} 3 \mathrm{~S} 3$ and $16 \mathrm{P} 4 \mathrm{~S} 4,9$ patches per each image).

Table 9 Parameters of the curvelet-based RBF SVM Classifier A for prostate cancer Gleason grade 3 versus grade 4

Table 10 Parameters of the CMRT-based RBF SVM Classifier B for prostate cancer Gleason grade 3 versus grade 4 ( $16 \mathrm{P} 3 \mathrm{~S} 3$ and $16 \mathrm{P} 4 \mathrm{~S} 4$, features from the average of 9 patches of an image).

Table 11 Parameters of the curvelet-based RBF SVM Classifier B for prostate cancer Gleason grade 3 versus grade 4 ( $16 \mathrm{P} 3 \mathrm{~S} 3$ and $16 \mathrm{P} 4 \mathrm{~S} 4$, features from the average of 9 patches of an image).

Table 12 Parameters of CMRT-based RBF SVM Classifier C for prostate cancer Gleason grade 3 versus grade 4 (34 P3 and 34 P4, average features from 9 patches of an image). 
Table 13 Parameters of the curvelet-based RBF SVM Classifier C for prostate cancer Gleason grade 3 and grade 4 (34 $\mathrm{P} 3$ and $34 \mathrm{P} 4$, average features from 9 patches of an image).....98 


\section{LIST OF FIGURES}

Figure 1 The Radon transform defined by the two parameters $\rho$ and $\theta$ specify the radial line of projection.

Figure 2 (a) A COMSFs of approximation order 2 and (b) corresponding cardinal wavelet functions.

Figure 3 Example of cardinal multiridgelets, (a) $\phi_{0, a, b, \theta}(x, y)$, (b) $\phi_{1, a, b, \theta}(x, y)$, (c) $\psi_{0, a, b, \theta}(x, y)$, (d) $\psi_{1, a, b, \theta}(x, y)$

Figure 4 The multiridgelet transform. Decomposition of one scale level is shown using multiwavelet transform, for multiplicity 2. Two channels are involved with low pass components (L1 and L2) and high pass components (H1 and H2)....

Figure 5 Characteristics of the scaling filters and wavelet filters of cardinal multiwavelet transform, (a) magnitude responses of the two scaling filters, (b) magnitude responses of the two wavelet filters, (c) group delay of scaling filters, (d) phase delay of scaling filters, (e) group delay of wavelet filters, and (f) phase delay of wavelet filters. 28

Figure 6 The wavelet coefficients of a signal $x(n)$, (a) signal $x(n)=\delta(n-60)$, (b) signal $x(n)=\delta(n-$ 64), (c) real coefficients of (a) using Daubechies-5 discrete wavelet transform, (d) real coefficients of (b) using Daubechies-5 discrete wavelet transform, (e) magnitude of complex coefficients of (a) using the cardinal multiwavelet transform, (f) magnitude of complex coefficients of (b) using the cardinal multiwavelet transform. 30

Figure 7 (a) a signal x(n), (b) its shifted version $x(n-4)$, (c) and (d) reconstruction of (a) and (b) from Daubechies-5 discrete wavelet transform at scale level 3, (e) and (f) reconstruction of (a) and (b) from dual-tree complex wavelet transform based on the cardinal multiwavelet transform at scale level 3 .

Figure 8 Sample 2-D images with square object and its Radon transform coefficients, (a) object aligned at center, (b) Radon coefficients of (a), (c) object translated, (d) Radon coefficients of (c), (e) high frequency coefficients of ridgelet transform of (a), (f) high frequency coefficients of ridgelet transform of (c), (g) magnitude of high frequency coefficients of the CMRT of (a), and (h) magnitude of high frequency coefficients of the CMRT of (c). 
Figure 9 Sample 2-D images with square object and its Radon transform coefficients, (a) object off from the center, (b) Radon coefficients of (a), (c) object rotated 45 degree, (d) Radon coefficients of (c), (e) high frequency coefficients of ridgelet transform of (a), (f) high frequency coefficients of ridgelet transform of $(\mathrm{c})$, (g) magnitude of high frequency scale coefficients of the CMRT of (a), and (h) magnitude of high frequency scale coefficients of the CMRT of (c).

Figure 10 CMRT decomposition to 3-scale and subband allocation with respect to scale and orientation.

Figure 11 List of images consists of the texture database of the study. Each image is $640 \times 640$ pixel.

Figure 12 Average retrieval rate of texture database, the number of closest matches increased from 15 to 100 with step size 5 .

Figure 13 Example of closest 15 similar matched images for a given query image from the image class "D24", (a) using Gabor wavelets, (b) using single ridgelets, (c) using curvelets, and (d) using CMRT, bold italic rank index indicates where the corresponding subsample is belonging to another class.

Figure 14 An example of simulated mura defects. An isotropic Gaussian function is placed at the center of the $256 \times 256$ sampled block of LCD display image displaying 50\% grayscale level: (a) mura with smaller radius and more intensity, and (b) mura with larger radius and less intensity. 57

Figure 15 CMRT coefficients 3-scale decomposed, merged all directions. .58

Figure 16 ROC curves of the mura defect classifiers based on (a) training sets and (b) validation sets.

Figure 17 Sample of test image for Mura defect detection. Placed two simulated blobs with different radius and intensity superimposed to the screen. White box indicates where the $256 \times 256$ pixel subimage taken to the algorithm.

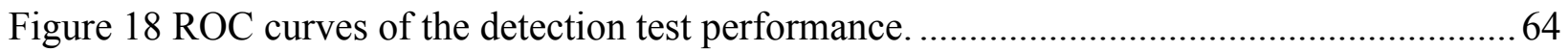

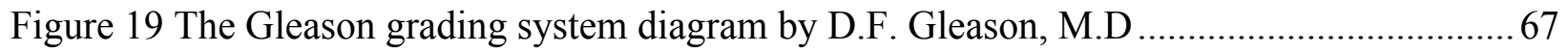

Figure 20 Examples of prostatic adenocarcinoma, (a) Gleason grade 2, (b) Gleason grade 3, (c) Gleason grade 4, and (d) Gleason grade 5, courtesy of WebPathology.com.....

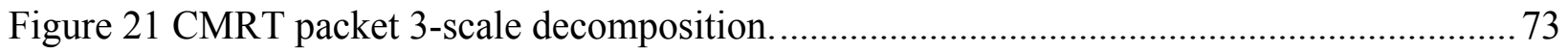

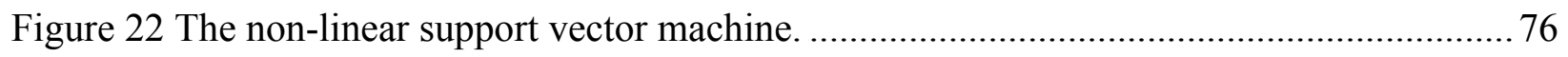

Figure 23 Subdividing prostate cancer images ............................................................. 78 
Figure 24 ROC curves of the validation test results of Classifier A for prostate cancer Gleason grade 3 versus grade 4 with PROPROC fitting method....................................................... 81

Figure 25 ROC curves of LOO validation test of Classifier B obtained by using PROPROC

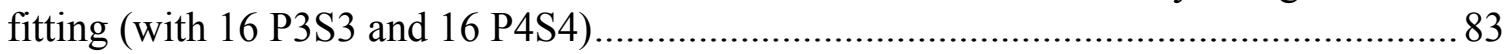

Figure 26 ROC curves of LOO validation test of Classifier C obtained by using PROPROC fitting (with $34 \mathrm{P} 3$ and $34 \mathrm{P} 4$ cases). 


\section{PREFACE}

I would like to thank my committee members: Dr. Boston, Dr. Chaparro, Dr. El-Jaroudi, Dr. Sun and especially advisor Dr. Li for their support and suggestion toward the completion of the research. I would also like to acknowledge Dr. Robert Veltri and Dr. Zhen Zhang for their providing of the Tissue Microarray dataset of prostate cancer histological images. It could not be accomplished without their support. I am also greatly thankful to Dr. Dev Chakraborty for his support and encouragement. Finally I want to thank my parents and brother in Republic of Korea for their love and endurance.

\section{BENE OMNIA FECIT}




\subsection{INTRODUCTION}

Since the advent of compactly supported orthogonal wavelets [1] and multiresolution signal decomposition [2], the multiresolution analysis has been widely applied to many science and engineering fields. One of the most important features of the wavelet transform is its capability of compacting the signal energy and thus efficiently representing functions that are smooth away from point singularities. Despite the fact that wavelets have had a wide impact on image processing, it has been noted that the standard wavelet transform has some limitations regarding their effectiveness in representing objects in images with highly anisotropic elements such as linear and curvilinear structures. Wavelets are non-geometrical and do not exploit regularity of edges, because wavelets rely on a dictionary of isotropic elements but do not describe anisotropic elements. Thus the standard 2-D wavelet transform of an image has poor directional selectivity. Most natural images exhibit curvilinear edges resulted from discontinuities across objects, which are called line and curve singularities. Thus the geometric feature extraction based upon the conventional 2-D wavelet transform would become less efficient in comparison to what can be achieved for 1-D signals.

An anisotropic geometric wavelet, named ridgelet, was proposed by Candés [3] and Donoho [4] to overcome the limitation of the standard 2-D wavelet transform, ridgelet transform is a kind of multiscale orientation-selective transform which takes the ridgelet as the basis element that has high directional selectivity; thus the ridgelet transform provides a key to 
the analysis of higher dimensional singularities. It processes the image data by first computing line integrals over different orientations and locations to obtain the Radon transform and then applying wavelet transform to the Radon transform coefficients. The Radon transform maps a line singularity of an input image into a point singularity along a certain radial direction [5], then the wavelet transform handles the point singularities in the Radon coefficients effectively. Hence, the ridgelet transform of 2-D image is very effective in representing objects with singularities along piecewise line segments.

The objective of this research is to improve the performance of ridgelet transform by introducing the multiwavelet transform [6] in the Radon domain to develop the multiridgelet transform. Multiwavelet basis possesses symmetry, orthogonality, compact support, and high approximation order simultaneously, which is not available to the single wavelet basis [7]. With the choice of the cardinal multiwavelet (with multiplicity equal to 2) [8] the resulting cardinal multiridgelet transform is both approximately shift-invariant and rotation-invariant. This will provide a significant advantage for image texture analysis where the cardinal multiridgelet transform is a dual-tree ridgelet transform and may be interpreted as a cardinal complex ridgelet transform. The magnitude of the complex transform coefficients can be applied in feature extraction for image texture classification.

With the developed cardinal multiridgelet transform, three applications have been explored: texture database retrieval, LCD mura defect detection, and prostate cancer histopathological image texture classification. In particular, the latter takes on the challenging task of computer-aided discrimination between two grades of prostate cancer that would be critical for predicting patient prognosis and affecting adequate treatment. With the image data Tissue Microarray (TMA) 471 from Johns Hopkins Medical School, cardinal multiridgelet- 
based texture features are extracted and a non-linear support vector machine has been developed to classify Gleason grade 3 and grade 4 of the prostate cancer.

The thesis is organized as follows. The cardinal multiridgelet transform and its properties are developed in Chapter 2. Chapter 3 describes experiments on two initial applications to texture data retrieval and LCD mura defect detection. The computer-aided classification of prostate cancer biopsy images is presented in Chapter 4. Chapter 5 summarizes the major contributions of the thesis and gives suggestions for future research. 


\subsection{CARDINAL MULTIRIDGELET TRANSFORM}

\subsection{BACKGROUND REVIEW}

Ridgelets [3] were developed to represent objects with highly anisotropic elements, e.g., lines, which the conventional 2-D wavelets do not exploit well. The ridgelet transform is a multiscale transform with frame elements such as scale and location parameters, and, in addition, having directional specificity; thus the ridgelet transform bears with anisotropic scaling principle. This background review section describes key principles of the ridgelet transform, so that it paves the way to extend to the multiridgelet transform.

\subsubsection{Radon transform}

The Radon transform definition used in many science and engineering fields can be found in [9]. If a bivariate function $f(x, y)$ has no preferred orientation, its Radon transform is described as the integral over the line $\rho=x \cos \theta+y \sin \theta$, written as

$$
R_{f(x, y)}(\rho, \theta)=\iint f(x, y) \delta(x \cos \theta+y \sin \theta-\rho) d x d y
$$


where $\delta(\bullet)$ is the Dirac delta function, $\rho \in \mathbf{R}$ is the perpendicular offset of a line with respect to the origin $(0,0)$, and $\theta \in[0, \pi)$ is the angle of a radial line of projection. That is, $R_{f(x, y)}(\rho, \theta)$ is the integral of the function $f(x, y)$ along lines $\rho=x \cos \theta+y \sin \theta$, as illustrated in Figure 1 .

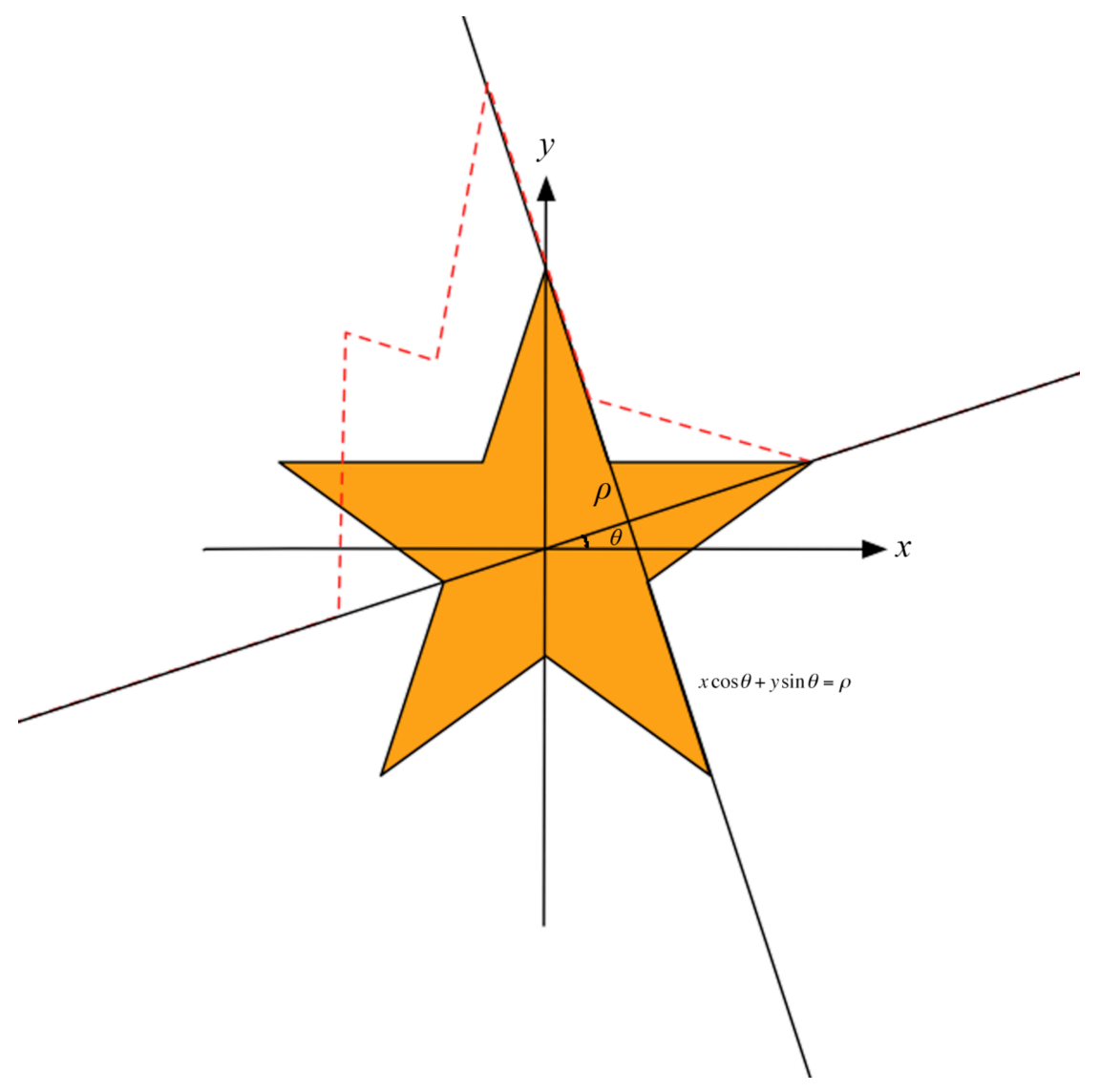

Figure 1 The Radon transform defined by the two parameters $\rho$ and $\theta$ specify the radial line of projection.

The Radon transform converts each of the line components into a peak positioned corresponding to the parameters of the lines, thus the task of finding the line singularities turns to finding the local peaks. 


\subsubsection{Ridgelet transform}

The 2-D continuous ridgelet transform in $\mathbf{R}^{2}$ is defined as follows. For a given smooth univariate wavelet function $\psi: \mathbf{R} \rightarrow \mathbf{R}$ satisfying the admissibility condition

$$
\int|\hat{\psi}| /|v|^{2} d v<\infty
$$

and vanishing mean

$$
\int \psi(t) d t=0
$$

define the bivariate ridgelet $\psi_{a, b, \theta}: \mathbf{R}^{2} \rightarrow \mathbf{R}$ by

$$
\psi_{a, b, \theta}(x, y)=\frac{1}{\sqrt{a}} \psi((x \cos \theta+y \sin \theta-b) / a)
$$

for each scale $a>0$, position $b \in \mathbf{R}$, and orientation $\theta \in[0,2 \pi)$. Therefore, a ridgelet is constant along lines $x \cos \theta+y \sin \theta=\rho$. Transverse to these ridges, it is a single wavelet.

The ridgelet transform of a given integrable bivariate function $f(x, y)$ is defined by

$$
R T_{f(x, y)}(a, b, \theta):=\left\langle f(x, y), \psi_{a, b, \theta}(x, y)\right\rangle=\iint f(x, y) \psi_{a, b, \theta}(x, y) d x d y
$$


It has the exact reconstruction

$$
f(x, y)=\int_{0}^{2 \pi} \int_{-\infty}^{\infty} \int_{0}^{\infty} R T_{f(x, y)}(a, b, \theta) \psi_{a, b, \theta}(x, y) \frac{d a}{a^{3}} d b \frac{d \theta}{4 \pi} .
$$

The ridgelet transform can be seen as the 1-D wavelet transform to the slices of the Radon transform. Let angular variable $\theta$ be constant and the line offset $\rho$ be varying, then the continuous ridgelet transform is the application of the 1-D single wavelet transform to a slice of the Radon transform coefficients,

$$
R T_{f(x, y)}(a, b, \theta)=\int \psi_{a, b}(\rho) R_{f(x, y)}(\rho, \theta) d \rho
$$

The single wavelet transform provides a sparse representation of line singularities that are converted to point singularities by the Radon transform. If a complex wavelet transform is applied then it becomes a complex ridgelet transform [10][11].

\subsubsection{Curvelet transform}

For 2-D images of objects, their edges are regarded as curved or piecewise linear singularities. In order to analyze local line or curve singularities, a natural way is to consider a partition of the image and then apply the ridgelet transform to the partitioned sub-images. This is the concept of the block ridgelet transform, named curvelet transform, which is the first-generation curvelet transform proposed by Candés and Donoho [12]. 
The idea of curvelets is to represent a curve as a superposition of functions of various lengths and widths obeying the parabolic scaling law width $\approx$ length $^{2}$. Algorithm of the firstgeneration curvelet transform is given as follows:

1. Subband decomposition. The image is filtered into subbands.

2. Smooth partitioning. Each subband is smoothly windowed into squares of appropriate scale.

3. Renormalization. Each resulting square is renormalized to unit scale.

4. Ridgelet Analysis. Each square is analyzed with the ridgelet transform.

However, the second-generation curvelets [13] does not use ridgelet transform. It is implemented with concentric squares and shears to have a tight frame expansion with lower redundancy. In this way the second-generation curvelet transform exhibits a simple and natural indexing structure with three parameters: scale, orientation, and location.

\subsubsection{Multiwavelet transform}

Multiwavelets were first proposed by Geronimo, Hardin, and Massopust (GHM) [6], and followed by Strang and Strela [14]. The GHM, a set of two scaling functions and two associated wavelets, has several attributes providing a bases for function representation to possess properties such as symmetry, orthogonality, compact support, and second-order approximation simultaneously. The ability of orthogonal wavelet bases to efficiently represent piecewise smooth functions is central to their success in estimation and compression. The compact support and approximation order are two key factors of wavelet bases, and there is a fundamental tradeoff between them. The generalization provided by bases with multiple scaling and wavelet functions permits greater flexibility in managing this trade-off. 
For scalar wavelet bases, the number of zero moments is an important measure of how well the wavelet transform compresses smooth signals. However, in the multiwavelet case, to guarantee the preservation/annihilation properties of the associated filter bank, it is not sufficient that the multiwavelet basis have zero moments. When the multiwavelet filter bank possesses the preservation/annihilation property for higher order input polynomials, it is said to have a higher balancing order.

The lack of balancing may be compensated by prefiltering the input [15]. However it may not be desirable due to some reasons. If the prefiltering step does not constitute an orthogonal transform, then the orthogonality of the multiwavelet transform is lost. The use of prefiltering increases the support of the basis functions if the prefilter has more than one nonzero coefficient. In the design of prefilters, it is desired that properties of the multiwavelet basis such as orthogonality, approximation order, and compact support be preserved as far as possible. One plausible solution is to consider the design of multiwavelets that preserve those properties with a specified approximation order, which is called balanced multiwavelets [16] where no prefiltering and postfiltering are required. 


\subsubsection{Cardinal multiwavelets}

A scaling function $\phi(t)$ satisfies the following conditions

$$
\phi(n)=\delta(n)=\left\{\begin{array}{cc}
1, & n=0 \\
0, & n= \pm 1, \pm 2, \ldots
\end{array}\right.
$$

as well as

$$
\int \phi(t) \phi(t-n) d t=\delta(n)
$$

and

$$
\phi(t)=\sqrt{2} \sum_{n} h(n) \phi(2 t-n)
$$

where $\{h(n)\}$ is the scaling filter, is called a cardinal orthogonal scaling function (COSF). 
The corresponding wavelet $\psi(t)$ satisfies

$$
\psi(t)=\sqrt{2} \sum_{n} g(n) \phi(2 t-n)
$$

where $\{g(n)\}$ is the wavelet filter. In this case the standard sampling theorem

$$
f(t)=\sum_{n} f\left(n / 2^{J}\right) \phi\left(2^{J} t-n\right), \forall f(t) \in V_{J}(\phi)
$$

holds for every COSFs [17].

Selesnick [8] proposed the cardinal multiwavelet transform that is possible to achieve cardinality, orthogonality, compact support, and approximation order $K>1$ simultaneously, which is not possible in the scalar wavelet transform. Let $\phi_{0}(t)$ and $\phi_{1}(t)$ be cardinal orthogonal multiscaling functions (COMSFs) with multiplicity equal to 2 . In this case

$$
\begin{aligned}
f(t) & =\sum_{n}\left(f(n) \phi_{0}\left(2^{J} t-n\right)+f\left(n+\frac{1}{2}\right) \phi_{1}\left(2^{J} t-n\right)\right), \forall f(t) \in V_{J}\left(\phi_{0}, \phi_{1}\right) \\
& =\sum_{n} f(n) \phi_{0}\left(2^{J} t-n\right)+\sum_{m} f(m) \phi_{1}\left(2^{J} t-\left(m-\frac{1}{2}\right)\right), m=n+\frac{1}{2} \\
& =\sum_{n} f(n) \phi_{0}\left(2^{J} t-n\right)+\sum_{m} f(m) \phi_{1}\left(2^{J}\left(t+2^{-(J+1)}\right)-m\right)
\end{aligned}
$$

holds, where $\phi_{0}(n / 2)=\delta(n)$ and $\phi_{1}(n / 2)=\delta(n-1)$. As a consequence, the COMSFs are a set of two COSFs with half-sample shift from each other. The scaling filters $h_{0}(n)$ and $h_{1}(n)$, and 
the corresponding wavelet filters $g_{0}(n)$ and $g_{1}(n)$ for order-2 balanced cardinal multiwavelet transform are given as follows

$$
\begin{aligned}
& h_{0}(n)=1 / \sqrt{2}(a, 0, b, 1, c, 0, d, 0, e, 0, f) \\
& h_{1}(n)=1 / \sqrt{2}(-f, 0, e, 0,-d, 1, c, 0,-b, 0, a) \\
& g_{0}(n)=1 / \sqrt{2}(-a, 0,-b, 1,-c, 0,-d, 0,-e, 0,-f) \\
& g_{1}(n)=1 / \sqrt{2}(f, 0,-e, 0, d, 1,-c, 0, b, 0,-a)
\end{aligned}
$$

where

$$
\begin{aligned}
& A=-1 / 8 \pm \sqrt{15} / 32 \\
& a=1 / 32 \\
& b=A+1 / 4 \\
& c=15 / 16 \\
& d=-2 A-1 / 4 \\
& e=1 / 32 \\
& f=A
\end{aligned}
$$

Figure 2 shows the plots of the scaling functions and wavelets. Note that here $\phi_{0}$ and $\phi_{1}$ of order-2 balanced cardinal functions are shifted, $\phi_{0}(3 / 2)=1$ and $\phi_{1}(4 / 2)=1[8]$. 

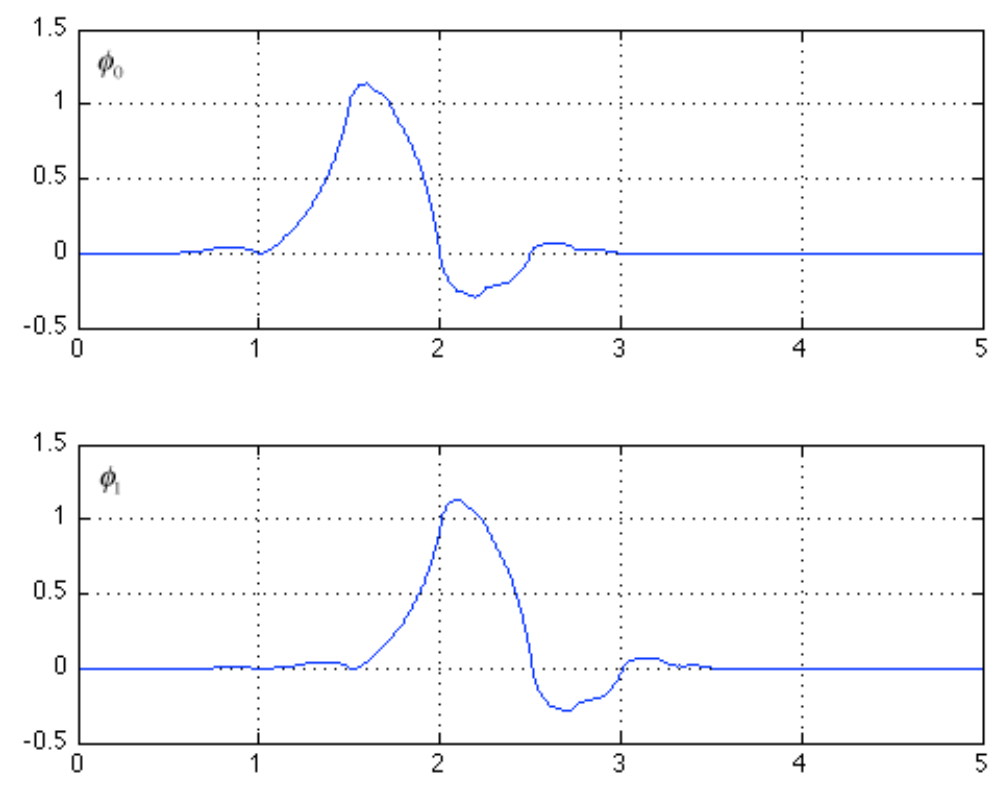

(a)
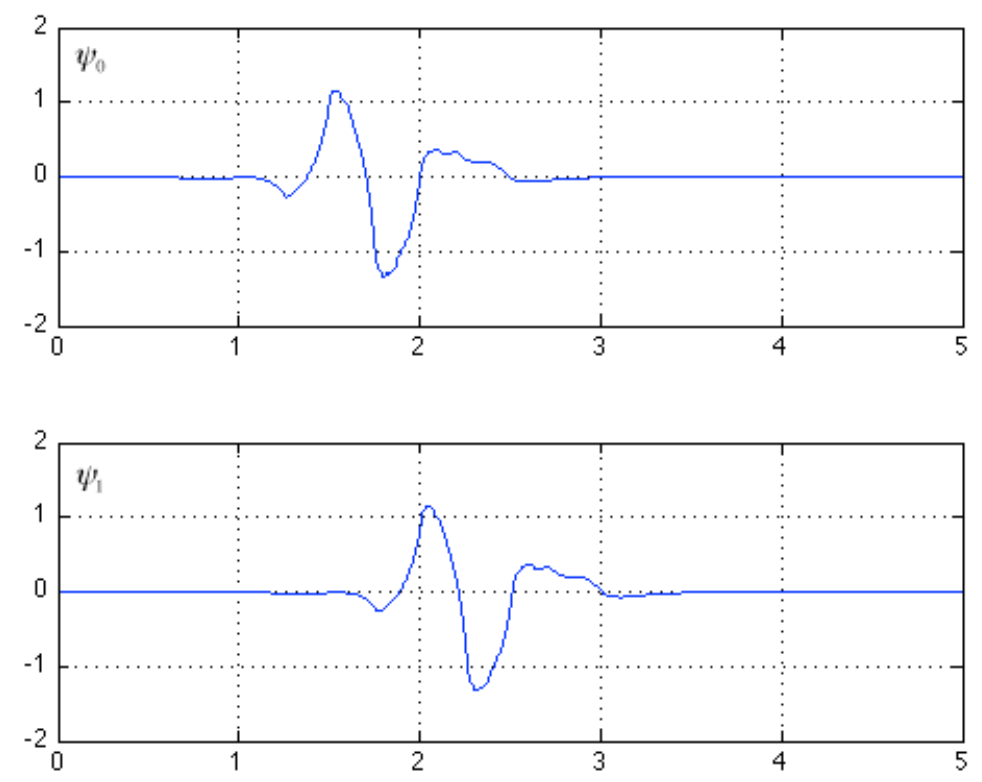

(b)

Figure 2 (a) A COMSFs of approximation order 2 and (b) corresponding cardinal wavelet functions. 


\subsection{MULTIRIDGELET TRANSFORM}

\subsubsection{Continuous multiridgelet transform}

For a given multiwavelet basis function $\underline{\psi}=\left[\psi_{0}(\rho), \psi_{1}(\rho)\right]^{T}$ (for multiplicity 2) and lines in polar representation $\rho=x \cos \theta+y \sin \theta$ in the $\mathrm{x}-\mathrm{y}$ plane, define the bivariate multiridgelet $\underline{\psi}_{a, b, \theta}$ by

$$
\underline{\psi}_{a, b, \theta}(x, y)=\frac{1}{\sqrt{a}} \underline{\psi}((x \cos \theta+y \sin \theta-b) / a)
$$

for each scale $a>0$, position $b$, and orientation $\theta$. Multiridgelet is constant along a line $x \cos \theta+y \sin \theta=\rho$. Transverse to these ridges, it is multiwavelet. Figure 3 is an example of cardinal multiscaling functions and corresponding multiridgelets. 


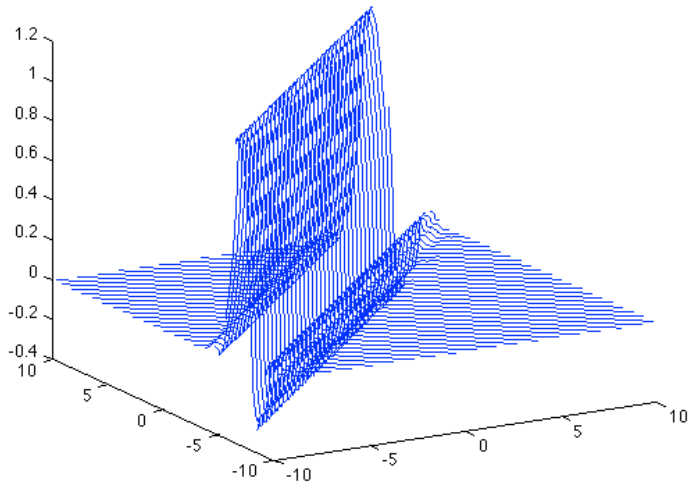

(a)

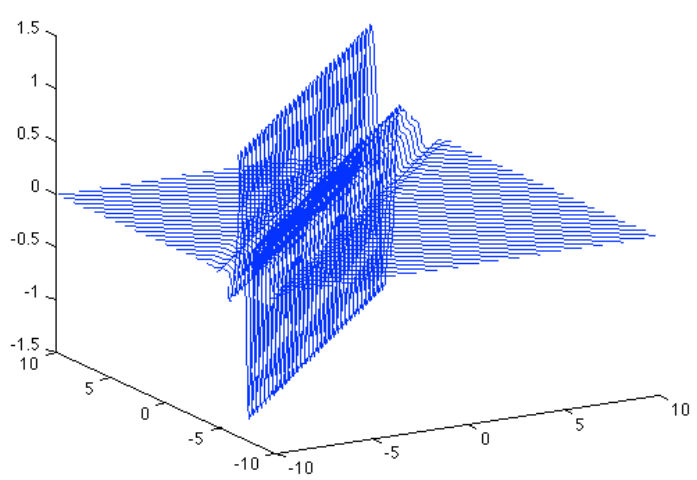

(c)

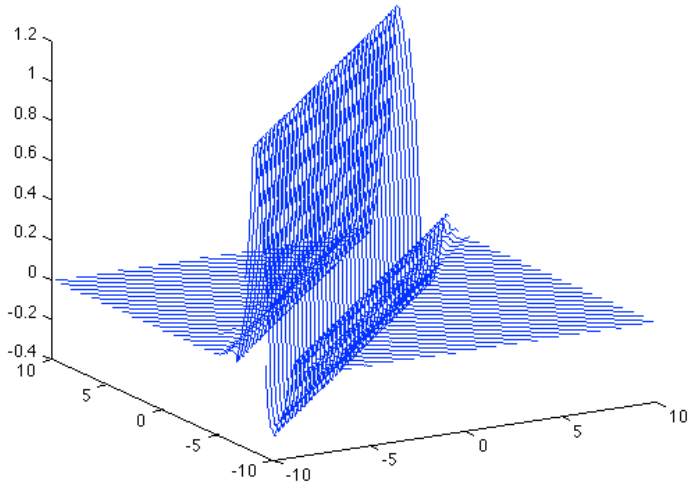

(b)

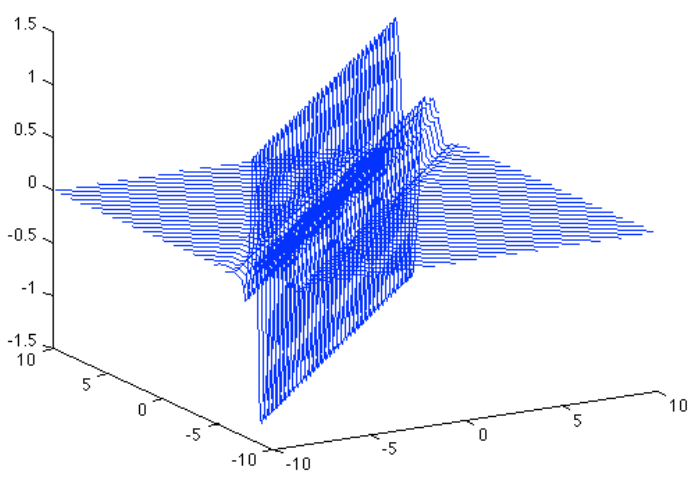

(d)

Figure 3 Example of cardinal multiridgelets, (a) $\phi_{0, a, b, \theta}(x, y)$, (b) $\phi_{1, a, b, \theta}(x, y)$,

(c) $\psi_{0, a, b, \theta}(x, y)$, (d) $\psi_{1, a, b, \theta}(x, y)$ 
The multiridgelet transform for a given integrable bivariate function $f(x, y)$ is defined by the inner product $\left\langle f(x, y), \psi_{a, b, \theta}(x, y)\right\rangle$,

$$
\underline{\operatorname{MRT}}_{f(x, y)}(a, b, \theta):=\left\langle f(x, y), \underline{\psi}_{a, b, \theta}(x, y)\right\rangle=\iint f(x, y) \underline{\psi}_{a, b, \theta}(x, y) d x d y
$$

The set of $\underline{M R T}_{f(x, y)}(a, b, \theta)$ are called the multiridgelet transform coefficients of $f(x, y)$.

The multiridgelet transform is seen as the 1-D multiwavelet transform along $\rho$ to the slices of the Radon transform of an image $f(x, y)$. It is first to compute the Radon transform $R_{f(x, y)}(\rho, \theta)$ and then to apply a 1-D multiwavelet transform to the slices of $R_{f(x, y)}(\rho, \theta)$ along $\rho$ for a particular angle $\theta$ and for different $\theta$ 's. Thus, the multiridgelet transform equation (4) can be rewritten as

$$
\underline{M R T}_{f(x, y)}(a, b, \theta)=\left\langle R_{f(x, y)}(\rho, \theta), \underline{\psi}_{a, b}(\rho)\right\rangle=\int R_{f(x, y)}(\rho, \theta) \underline{\psi}_{a, b}(\rho) d \rho
$$

Especially, if the 1-D multiwavelet transform used is the cardinal multiwavelet transform, it is the cardinal multiridgelet transform (CMRT). 


\subsubsection{Multiresolution analysis of multiridgelet transform}

For a nesting of the spanned space defined as

$$
V_{j} \subset V_{j+1}, \forall j \in \mathbf{Z} \text { with } V_{-\infty}=\{0\}, V_{\infty}=L^{2}
$$

the subspaces have to satisfy a natural scaling condition

$$
f(t) \in V_{j}, f(2 t) \in V_{j+1}
$$

From the wavelet theory, the scaling space $V_{j}$ is given by

$$
V_{j}=\underset{k}{\operatorname{Span}}\left\{\phi_{0}\left(2^{j} t-k\right), \phi_{1}\left(2^{j} t-k\right)\right\}, \forall k \in \mathbf{Z}
$$

Also define the wavelet spanned subspaces $W_{i}$ such that

$$
V_{1}=V_{0} \oplus W_{0}, V_{2}=V_{1} \oplus W_{1}=V_{0} \oplus W_{0} \oplus W_{1}, \cdots .
$$


For the cardinal orthogonal multiscaling function (COMSF) $\phi_{0}(t)$ and $\phi_{1}(t)$, they satisfy the condition

$$
\phi_{0}(n / 2)=\delta(n), \phi_{1}(n / 2)=\delta(n-1)
$$

Following is the multiscaling function under dyadic dilations and translations

$$
\underline{\phi}_{j, k}(t)=2^{j / 2} \underline{\phi}\left(2^{j} t-k\right) .
$$

Similarly, for the multiwavelet functions

$$
\underline{\psi}_{j, k}(t)=2^{j / 2} \underline{\psi}\left(2^{j} t-k\right)
$$


Since the $\underline{\phi(t)}$ is the COMSF, the scaling and wavelet functions satisfy a matrix dilation and wavelet equations

$$
\begin{aligned}
& \underline{\phi}(t)=\sqrt{2} \sum_{n} \underline{H}(n) \underline{\phi}(2 t-n) \\
& \underline{\psi}(t)=\sqrt{2} \sum_{n} \underline{G}(n) \underline{\phi}(2 t-n)
\end{aligned}
$$

where

$$
\begin{aligned}
& \underline{H}(n)=\left[\begin{array}{ll}
h_{0}(2 n) & h_{0}(2 n+1) \\
h_{1}(2 n) & h_{1}(2 n+1)
\end{array}\right] \\
& \underline{G}(n)=\left[\begin{array}{ll}
g_{0}(2 n) & g_{0}(2 n+1) \\
g_{1}(2 n) & g_{1}(2 n+1)
\end{array}\right]
\end{aligned}
$$

where $h_{0}(n)$ and $h_{1}(n)$ are two scaling filters, and $g_{0}(n)$ and $g_{1}(n)$ are two wavelet filters, $n \in \mathbf{Z}$. Also, $\phi_{0}(t)$ and $\phi_{1}(t)$ are orthogonal to their integer shifts

$$
\int \phi_{i}(t) \phi_{j}(t-n) d t=\delta(i-j) \delta(n), i, j \in\{0,1\}
$$

and the wavelet functions satisfy

$$
\int \psi_{i}(t) d t=0, i, j \in\{0,1\} .
$$




\subsubsection{Discrete multiridgelet transform}

For orthogonal scaling and wavelet spaces,

$$
V_{0} \subset V_{1} \subset V_{2} \subset \cdots \subset L^{2}
$$

and

$$
L^{2}=V_{0} \oplus W_{0} \oplus W_{1} \oplus \cdots
$$

From (5), for any 2-D image $f(x, y)$, its Radon transform $R_{f(x, y)}(\rho, \theta) \in L^{2}([0, \pi) \times \mathbf{R})$ can be expressed as a series of expansion in terms of the cardinal multiscaling function and multiwavelet function (multiplicity 2 ) with respect to the translation $k$ in $\rho$, i.e., as the inverse of its cardinal multiridgelet transform coefficients,

$$
R_{f(x, y)}(\rho, \theta)=\sum_{k} \underline{c}_{0, k, \theta} \underline{\phi}_{0, k}(\rho)+\sum_{j=0}^{\infty} \sum_{k} \underline{d}_{j, k, \theta} \underline{\psi}_{j, k}(\rho)
$$

where $\underline{\phi}_{j, k}(\rho)=\left[\begin{array}{c}\phi_{0, j, k}(\rho) \\ \phi_{1, j, k}(\rho)\end{array}\right]$ and $\underline{\psi}_{j, k}(\rho)=\left[\begin{array}{c}\psi_{0, j, k}(\rho) \\ \psi_{1, j, k}(\rho)\end{array}\right]$ are the discrete forms of $\underline{\phi}_{a, b}(\rho)$ and $\underline{\psi}_{a, b}(\rho)$ respectively, with scale $a=2^{j}$ and shift $b=k$, 


$$
\begin{aligned}
& c_{0, j, k, \theta}=\int R_{f(x, y)}(\rho, \theta) \phi_{0, j, k}(\rho) d \rho, \\
& c_{1, j, k, \theta}=\int R_{f(x, y)}\left(\rho+\frac{1}{2}, \theta\right) \phi_{1, j, k}(\rho) d \rho, \\
& d_{0, j, k, \theta}=\int R_{f(x, y)}(\rho, \theta) \psi_{0, j, k}(\rho) d \rho, \\
& d_{1, j, k, \theta}=\int R_{f(x, y)}\left(\rho+\frac{1}{2}, \theta\right) \psi_{1, j, k}(\rho) d \rho,
\end{aligned}
$$

are the cardinal multiridgelet transform coefficients. Figure 4 illustrates the flow of the multiridgelet decomposition. A given image $f(x, y)$ is transformed via the Radon transform, and it is fed to the 1-D multiwavelet transform with respect to each radial line $\rho$. Note that here the same signal is fed into both channels for multiwavelet decomposition and no prefiltering is needed. The example shown at the upper right corner in Figure 4 is the Radon transform for a radial angle $\theta_{0}, R_{f(x, y)}\left(\rho, \theta_{0}\right)$ is one of the row element in Radon coefficients array. For computation purpose, the generalized Radon transform based on slant stacks is implemented for discretization [9]. 


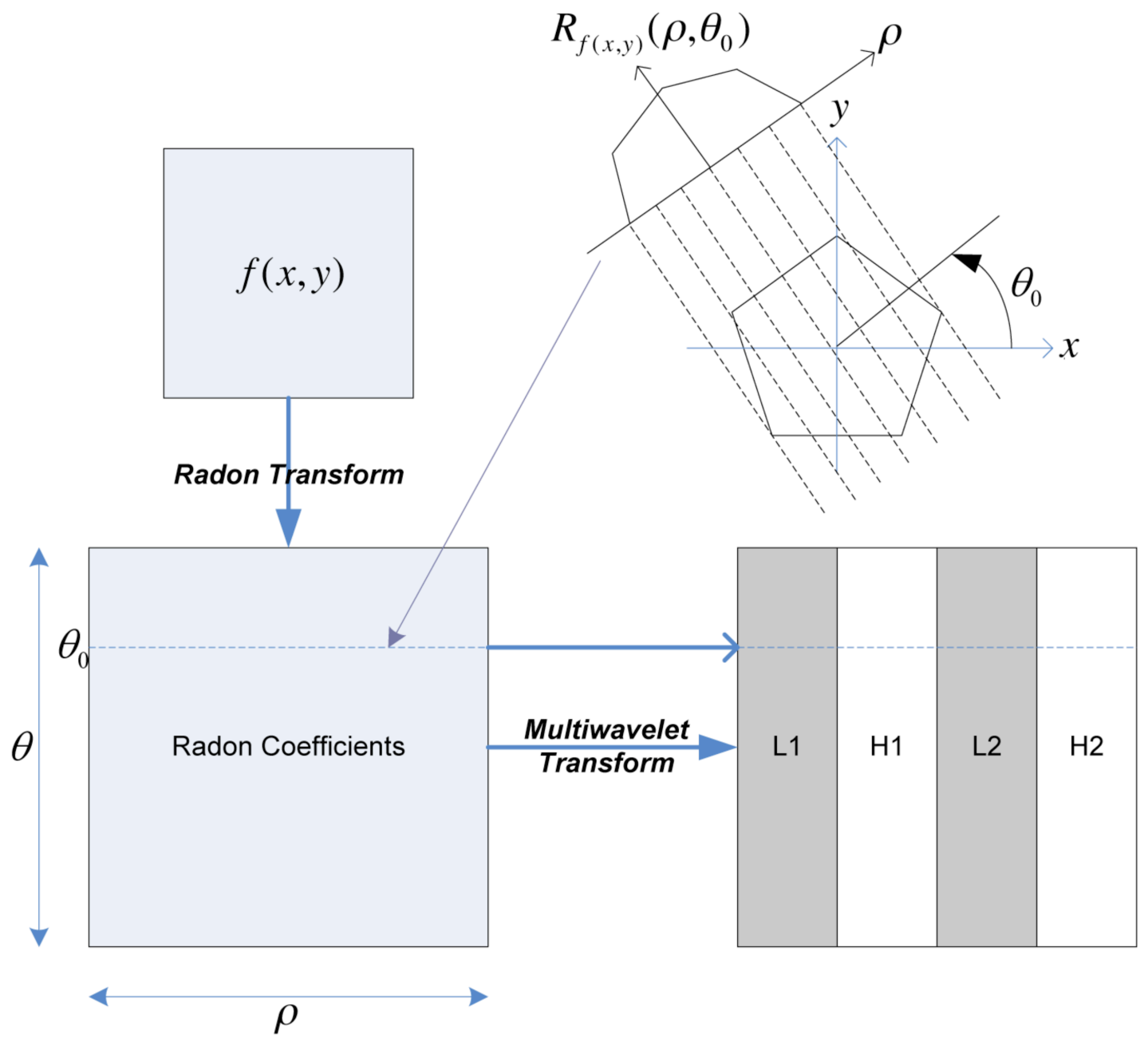

Figure 4 The multiridgelet transform. Decomposition of one scale level is shown using multiwavelet transform, for multiplicity 2 . Two channels are involved with low pass components (L1 and L2) and high pass components (H1 and H2). 


\subsection{PROPERTIES OF CARDINAL MULTIRIDGELET TRANSFORM}

As discussed in the earlier sections, the cardinal multiridgelet transform (CMRT) is composed of the Radon transform followed by the 1-D cardinal multiwavelet transform. Naturally it is expected to possess advantages from both transforms. We will examine three advantageous properties of the CMRT, in particular, with respect to its applications to texture analysis. They are cardinality, approximate translation invariance, and approximate rotation invariance.

\subsubsection{Cardinality}

Based upon the Shannon sampling theorem, it is clear that the sinc function satisfies the condition of cardinal orthogonal scaling function (COSF), and the same for the Haar wavelets. However, the sinc function is not of compact support, and the Haar function has only approximation order-1. Xia and Zhang [17] demonstrated that there exists a COSF that exhibits a higher approximation order as well as compact support. When $f(t) \in V_{J+1}(\phi)$, the aliasing error is the difference [18]

$$
e(t):=f(t)-\sum_{n} f\left(n / 2^{J}\right) \phi\left(2^{J} t-n\right)
$$

For estimating this error numerically, $f(t)$ is not necessarily in $V_{J+1}(\phi)$ [17]. For the case expressed by Equation (1), the estimated aliasing error of COSFs is none. It is also true for the 
expression given by the cardinal multiscaling function (COMSF) in Equation (2). The fact that the COMSFs $\phi_{0}(t)$ and $\phi_{1}(t)$ are half-sample shift from each other leads to achieve nice properties that will be described in the next subsections.

\subsubsection{Approximate translation invariance}

Since the real-valued wavelets are bandpass functions, the wavelet transform coefficients tend to oscillate around singularities, therefore a small shift of the signal will result in substantial changes of the wavelet coefficients around singularities. It is also carried through to the single ridgelet transform. A most practical solution to this problem is to use the complex-valued wavelets [19]. By applying complex wavelet transform, the magnitude of the complex coefficients does not oscillate but provide a smooth positive envelope that preserves shift invariant property.

A complex-valued wavelet $\psi_{c}(t)$ is described by

$$
\psi_{c}(t)=\psi_{r}(t)+j \cdot \psi_{i}(t)
$$

where $\psi_{r}(t)$ is the real part and $j \psi_{i}(t)$ is the imaginary part. 
Decomposing a signal by complex wavelet transform, we obtain the complex wavelet transform coefficients

$$
d_{c}(n)=d_{r}(n)+j \cdot d_{i}(n)
$$

with magnitude

$$
\left|d_{c}(n)\right|=\sqrt{\left|d_{r}(n)\right|^{2}+\left|d_{i}(n)\right|^{2}}
$$

and phase

$$
\angle d_{c}(n)=\arctan \left(\frac{d_{i}(n)}{d_{r}(n)}\right) .
$$

Kingsbury [20] proposed an application of two real-valued wavelet transforms in parallel with filters forming a Hilbert transform pair, where one wavelet is the Hilbert transform of the other. Selesnick [21] showed that for two orthogonal wavelets to form a Hilbert transform pair, the scaling filters $\left\{h_{0}(n)\right\}$ and $\left\{h_{1}(n)\right\}$ should be offset by a half sample, rewritten in terms of the magnitude and phase conditions, they are

$$
\begin{aligned}
& \left|H_{1}(\omega)\right|=\left|H_{0}(\omega)\right|, \\
& \angle H_{1}(\omega)=\angle H_{0}(\omega)-\frac{1}{2} \omega .
\end{aligned}
$$


However, such a system is not realizable with an ideal fractional delay filter, because if $h_{0}(n)$ is FIR, then $h_{1}(n)$ would be of infinite length. If both $h_{0}(n)$ and $h_{1}(n)$ are FIR filters and if the above two conditions are approximately satisfied for nearly the whole frequency interval, then the wavelets $\psi_{r}(t)$ and $\psi_{i}(t)$ would be an approximate Hilbert transform pair. A number of filter design methods have been studied such as biorthogonal solution [22][23], orthonormal allpass solution [24][25], and orthonormal IIR filter solution [26]. Among those studies, the equal magnitude response and half-sample phase delay between the filter responses are the primary tools for considering Hilbert transform pairs. 
The observation that the two scaling functions and the two wavelet functions of the cardinal multiwavelet transform are half-sample shifted leads to our inquiry into the possibility of being an approximate Hilbert transform pair under certain situations, and some interesting characteristics were explored by computational results. The two scaling filters, $\left\{h_{0}(n)\right\}$ and $\left\{h_{1}(n)\right\}$, of the cardinal multiwavelet transform have almost the same magnitude responses, $\left|H_{0}(\omega)\right|$ and $\left|H_{1}(\omega)\right|$, while the two filters have half-sample group delay difference as well as half-sample phase delay difference in their pass-band. Similarly, the wavelet filters, $\left\{g_{0}(n)\right\}$ and $\left\{g_{1}(n)\right\}$, of the cardinal multiwavelet transform also have almost the same magnitude responses, $\left|G_{0}(\omega)\right|$ and $\left|G_{1}(\omega)\right|$, and have half-sample group delay difference and half-sample phase delay difference in their pass-band as well. Figure 5 illustrates the characteristics of two scaling filters and wavelet filters of the cardinal multiwavelet transform, where the frequency $\omega$ is normalized by $\pi$. Therefore, we conclude here that the cardinal multiwavelet basis may be utilized as an approximate Hilbert transform pair. 


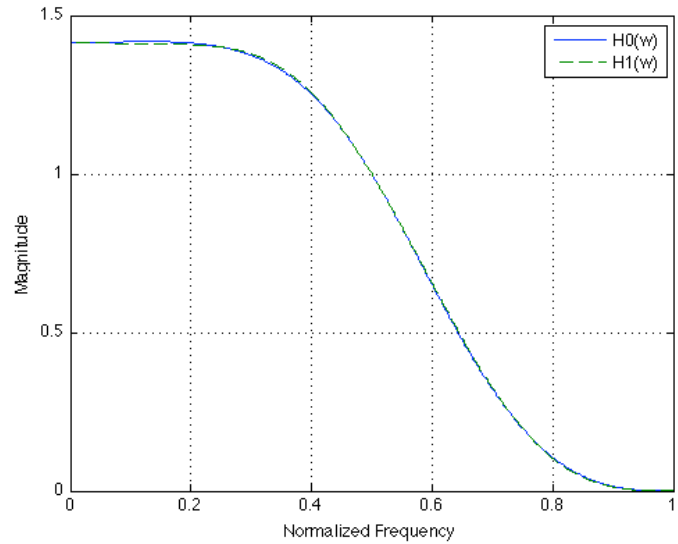

(a)

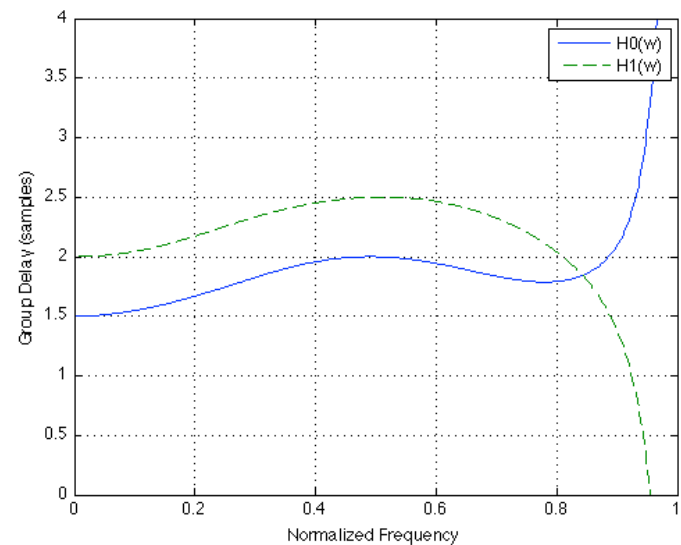

(c)

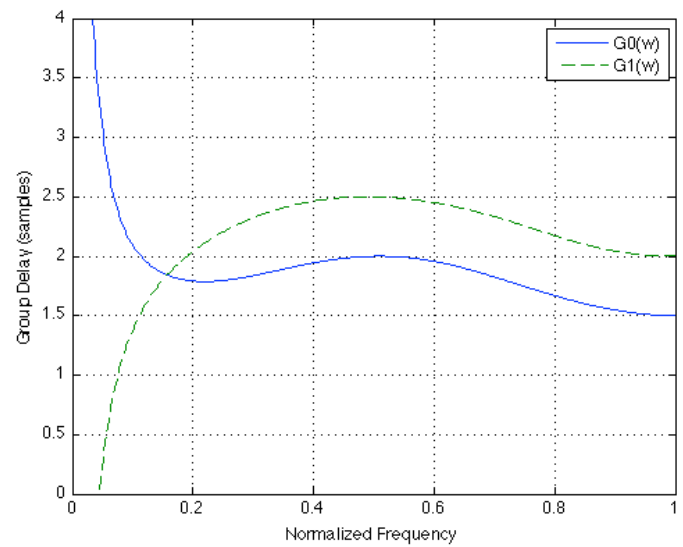

(e)

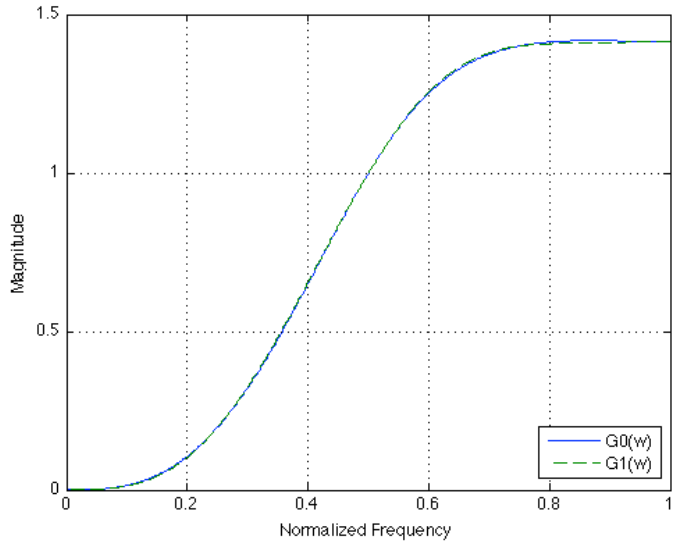

(b)

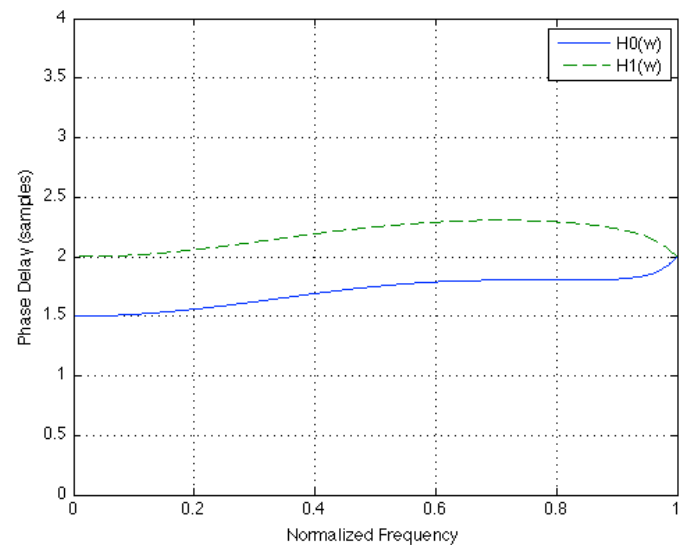

(d)

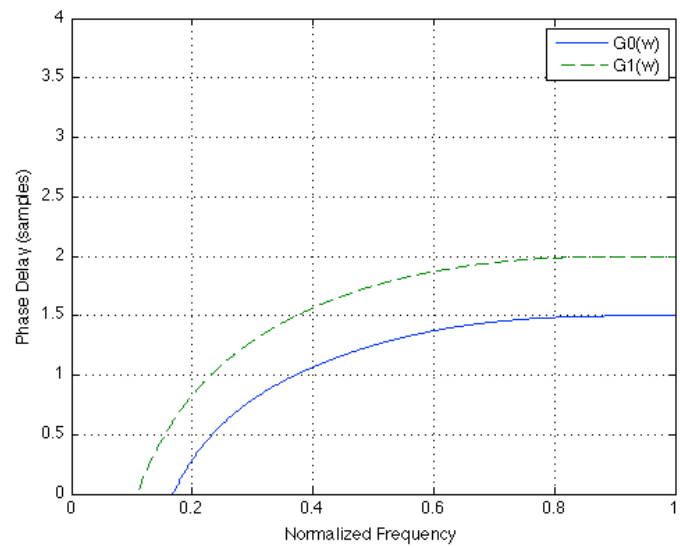

(f)

Figure 5 Characteristics of the scaling filters and wavelet filters of cardinal multiwavelet transform, (a) magnitude responses of the two scaling filters, (b) magnitude responses of the two wavelet filters, (c) group delay of scaling filters, (d) phase delay of scaling filters, (e) group delay of wavelet filters, and (f) phase delay of wavelet filters. 
The effect of adaptation of the two cardinal multiwavelet basis as an almost approximate Hilbert transform pair is further examined by the replication of the demonstration shown in [27]. Figure 6 illustrates the magnitude of complex coefficients of the cardinal multiwavelet transform. With the dual-tree implementation, each pair of the 2-channel coefficients is combined in the form of the square root of sum of squares. This would be equivalent to considering the cardinal multiwavelet transform as providing a complex wavelet transform. The total energy at the third scale level decomposition is nearly constant regardless of the shift of input signal. In contrast, the Daubechies-5 discrete wavelet transform coefficients show substantial differences in regard to the shift of the input signal. 

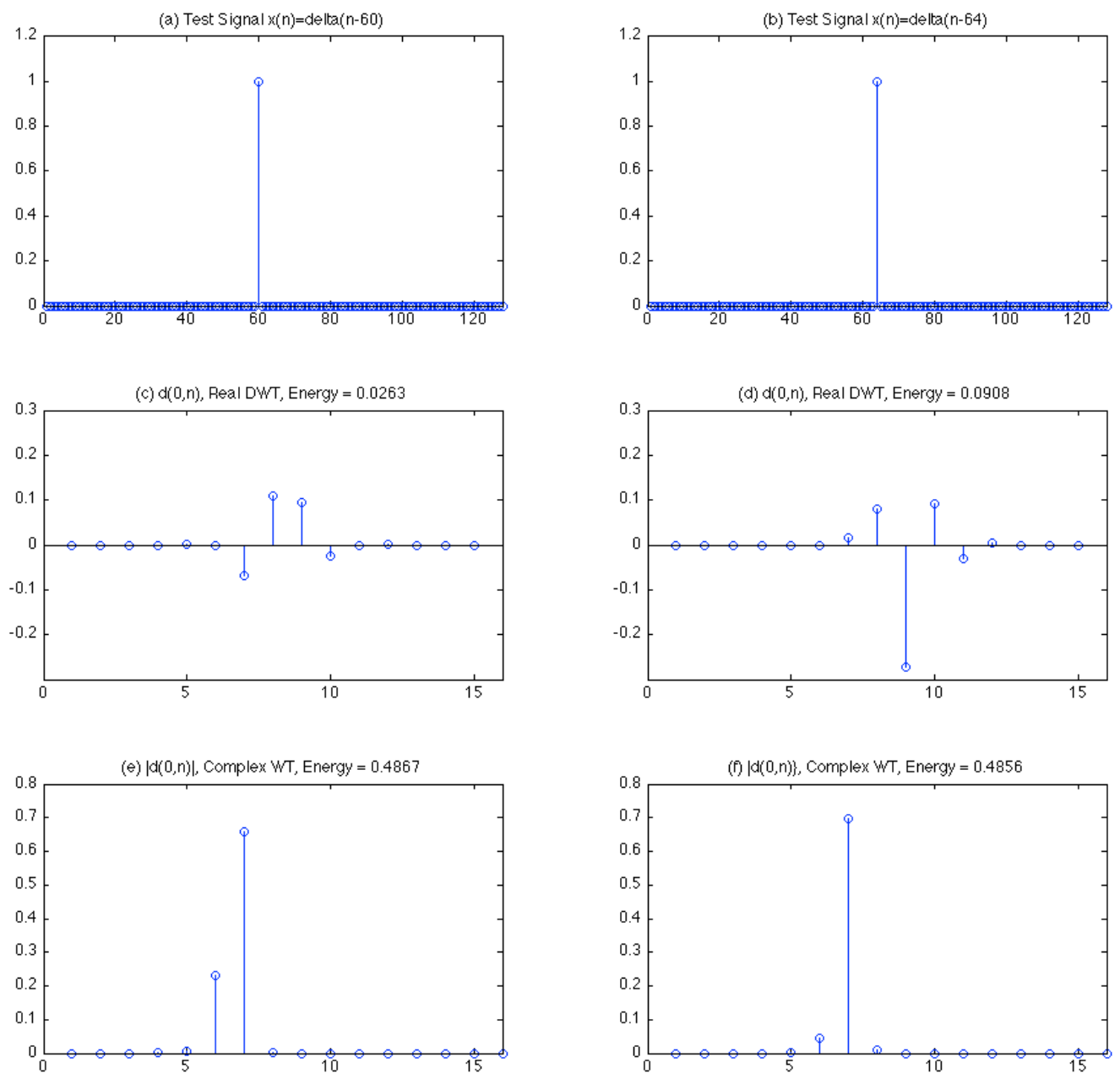

Figure 6 The wavelet coefficients of a signal $x(n)$, (a) signal $x(n)=\delta(n-60)$, (b) signal $x(n)=\delta(n-64)$, , c) real coefficients of (a) using Daubechies-5 discrete wavelet transform, (d) real coefficients of (b) using Daubechies-5 discrete wavelet transform, (e) magnitude of complex coefficients of (a) using the cardinal multiwavelet transform, (f) magnitude of complex coefficients of (b) using the cardinal multiwavelet transform. 
Another example considered is a rectangular pulse signal, its shifted version, and their corresponding reconstructions from the respective transform coefficients at the scale level 3 as shown in Figure 7. It demonstrates that the dual-tree based cardinal multiwavelet transform maintains its shape of the high-frequency component regardless the signal shift, thus illustrating its almost shift invariance characteristic. 

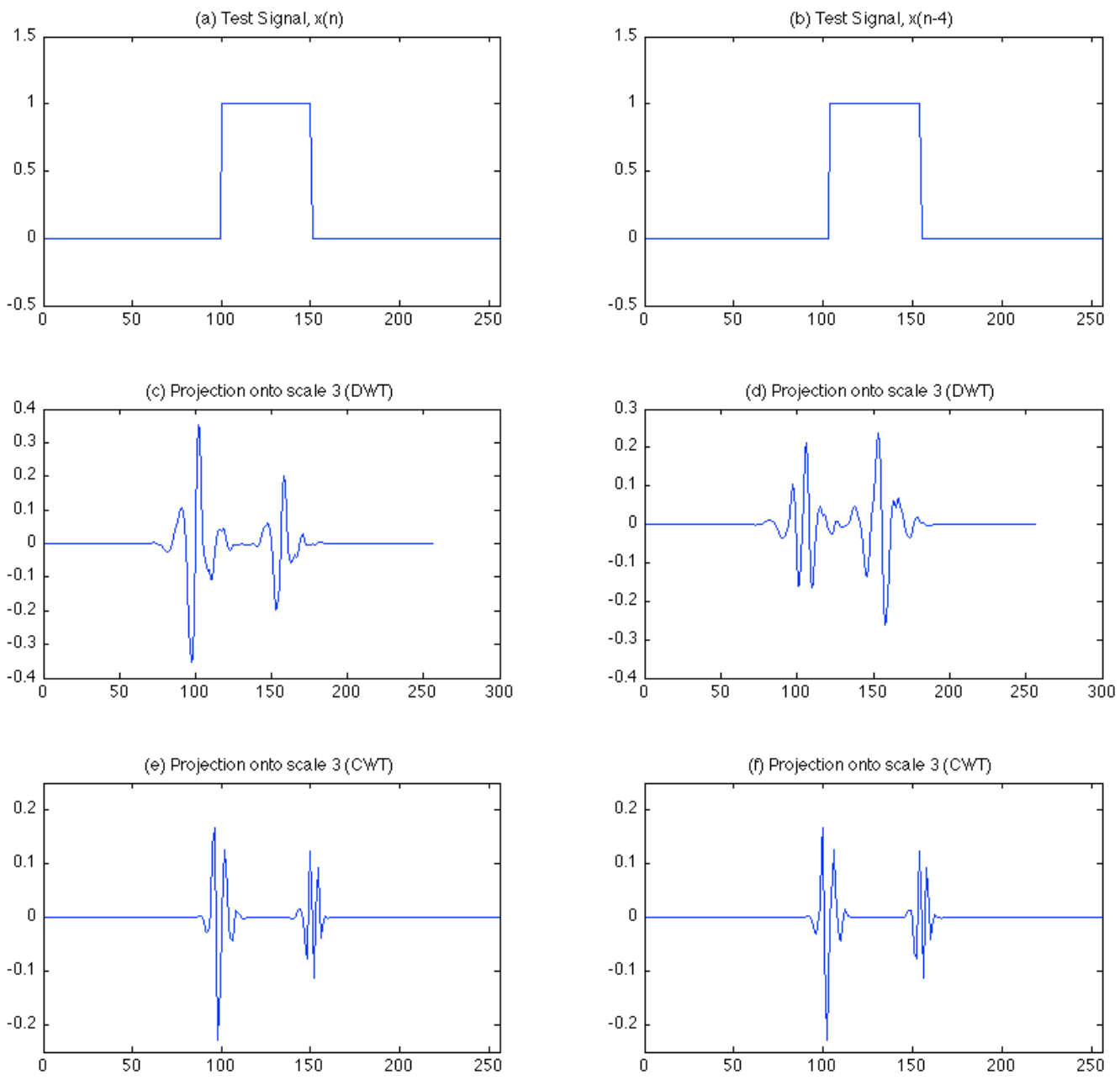

Figure 7 (a) a signal $x(n),(b)$ its shifted version $x(n-4),(c)$ and (d) reconstruction of (a) and (b) from Daubechies-5 discrete wavelet transform at scale level 3, (e) and (f) reconstruction of (a) and (b) from dual-tree complex wavelet transform based on the cardinal multiwavelet transform at scale level 3. 
Because of the almost shift invariance of the cardinal multiwavelet transform, the coefficients of CMRT may also be considered as complex coefficients as used in complex ridgelet which has nearly shift invariant characteristics [10][11], so that

$$
C M R T_{C, f(x, y)}(a, b, \theta)=C M R T_{0, f(x, y)}(a, b, \theta)+j \cdot C M R T_{1, f(x, y)}(a, b, \theta)
$$

where $C M R T_{0}$ is the coefficient from the first channel regarded as the real part and $C M R T_{1}$ is the coefficients from the second channel regarded as the imaginary part. Then,

$$
\left|C M R T_{C, f(x, y)}(a, b, \theta)\right|=\sqrt{\left|C M R T_{0, f(x, y)}(a, b, \theta)\right|^{2}+\left|C M R T_{1, f(x, y)}(a, b, \theta)\right|^{2}}
$$

and

$$
\angle C M R T_{C, f(x, y)}(a, b, \theta)=\arctan \left(\frac{C M R T_{1, f(x, y)}(a, b, \theta)}{C M R T_{0, f(x, y)}(a, b, \theta)}\right),
$$


The magnitude array of the CMRT coefficients also has the almost shift invariant property. Suppose that a square object $f(x, y)$ is translated from the center coordinates by $\zeta$ pixels along the $y$ axis

$$
f^{\prime}(x, y)=f(x, y-\zeta)
$$

as shown in Figure 8(c). Its Radon transform is given by

$$
\begin{aligned}
R_{f^{\prime}(x, y)}(\rho, \theta) & =\iint f(x, y) \delta(x \cos \theta+(y+\xi) \sin \theta-\rho) d x d y \\
& =\iint f(x, y) \delta(x \cos \theta+y \sin \theta-(\rho-\zeta \sin \theta)) d x d y
\end{aligned}
$$

which implies that the translation of the object is reflected as a sinusoidal shift in the $\rho$ variable in the Radon transform. When there is no translation $(\zeta=0)$ as shown in Figure 8(a), its Radon coefficients are aligned at the center as shown in Figure 8(b). When there is a translation shown in Figure 8(c), its Radon coefficients do appear as a twisted beam shape shown in Figure 8(d). Indeed, the amplitude of the sinusoidal twist in Radon domain is equal to the radial distance of translation from the center of the image.

Because the single ridgelet transform is translation sensitive, the high frequency coefficients of the single ridgelet coefficients of Figure 8(a) and Figure 8(c) are shown in Figure 8(e) and Figure 8(f), respectively. They do not show the same amplitude. It is observed that some positive peaks of coefficients in Figure 8(e) are inverted to negative peaks in Figure 8(f), and vice versa. On the other hand, the magnitudes of high frequency coefficients of the CMRT of the original and the translated object given in Figure 8(g) and Figure 8(h), respectively, show 
that the magnitude peaks are not changed by the shift of an object. This supports assumption that the CMRT is almost translation invariant. 


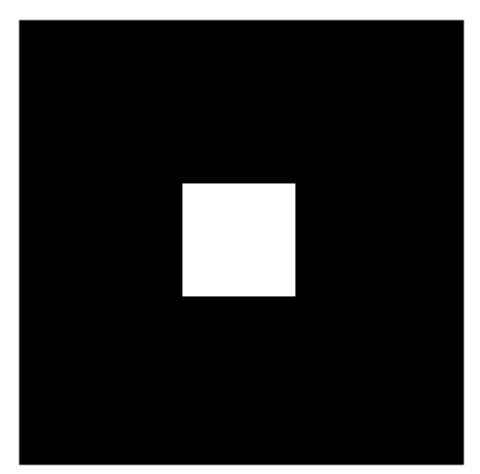

(a)

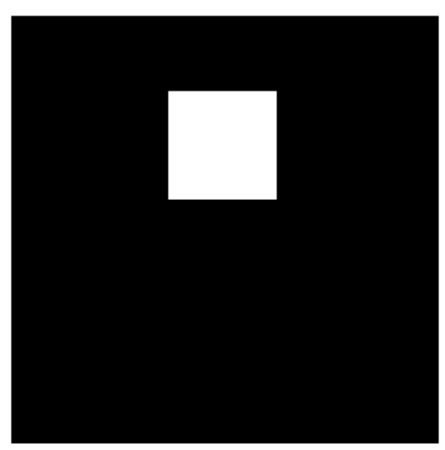

(c)

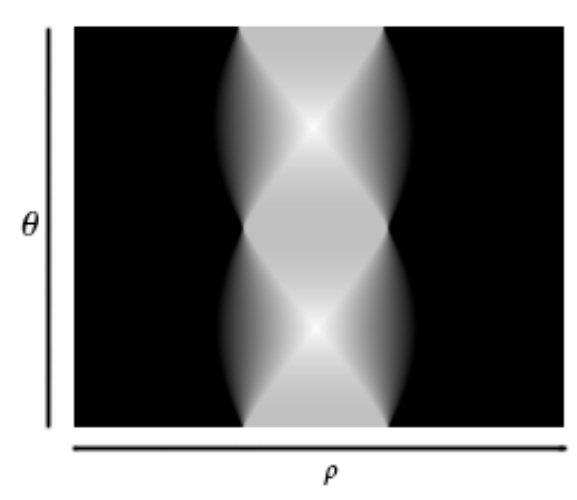

(b)

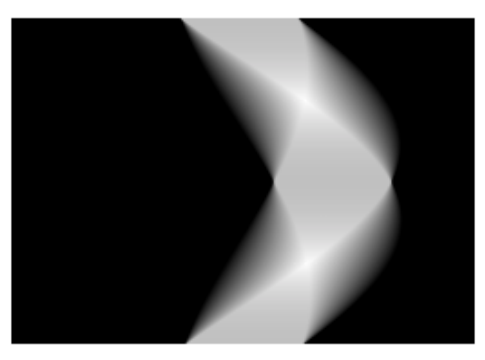

(d) 


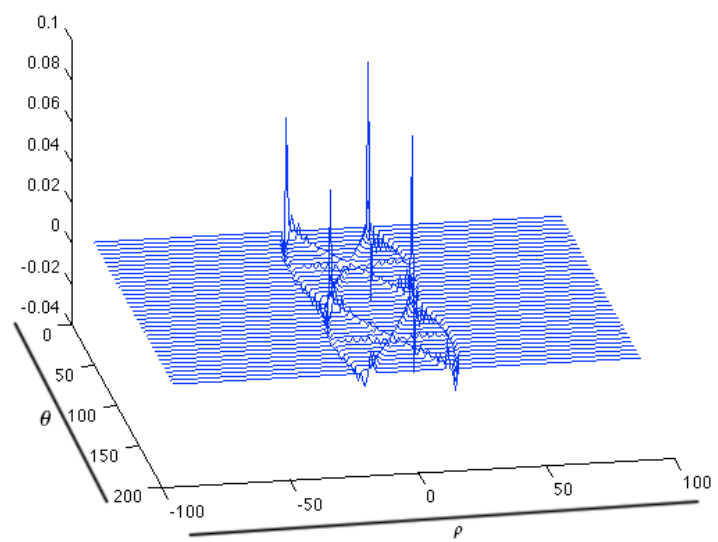

(e)

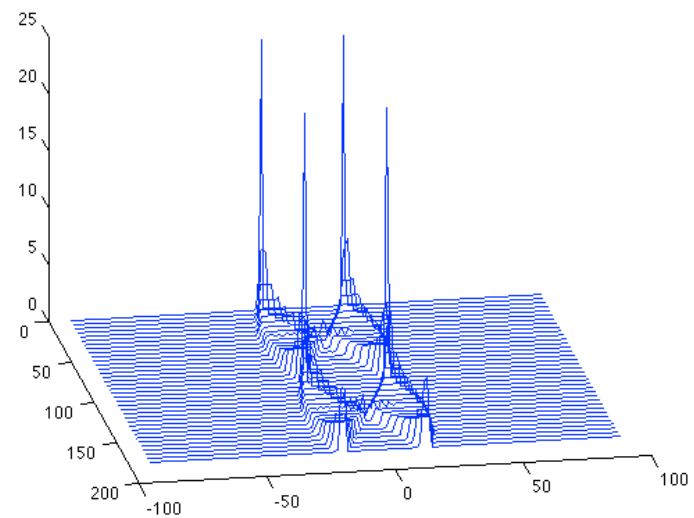

(g)

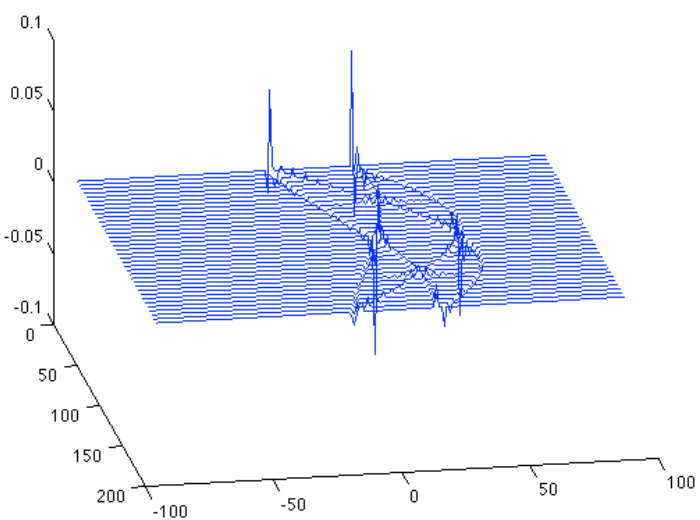

(f)

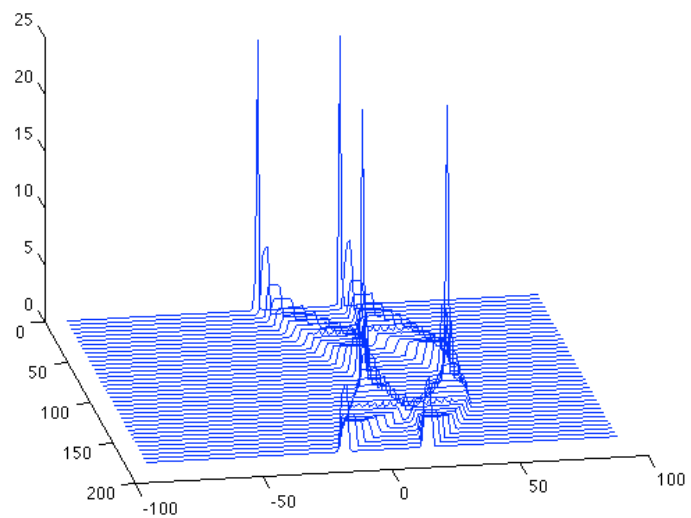

(h)

Figure 8 Sample 2-D images with square object and its Radon transform coefficients, (a) object aligned at center, (b) Radon coefficients of (a), (c) object translated, (d) Radon coefficients of (c), (e) high frequency coefficients of ridgelet transform of (a), (f) high frequency coefficients of ridgelet transform of (c),

(g) magnitude of high frequency coefficients of the CMRT of (a), and (h) magnitude of high frequency coefficients of the CMRT of (c). 


\subsubsection{Approximate rotation invariance}

One of the advantages inherited from the Radon transform is its rotation invariant property. Let $f(x, y)$ be the original image, its Radon transform is $R_{f(x, y)}(\rho, \theta)$. Suppose that the image is rotated by angle $\varphi, f^{\prime \prime}(x, y)$, then $R_{f^{\prime \prime}(x, y)}(\rho, \theta)$ is the integral of the object $f(x, y)$ over the line $\rho=x \cos (\theta+\varphi)+y \sin (\theta+\varphi)$, which leads to a circular shift of the Radon transform $R_{f(x, y)}(\rho, \theta)$ of the origin of $f(x, y)$,

$$
\begin{aligned}
R_{f^{\prime \prime}(x, y)}(\rho, \theta) & =\iint f(x, y) \delta(x \cos (\theta+\varphi)+y \sin (\theta+\varphi)-\rho) d x d y \\
& =R_{f(x, y)}(\rho, \theta+\varphi)
\end{aligned}
$$

Therefore, $R_{f^{\prime \prime}(x, y)}(\rho, \theta)$ is a circular shift by angle $\varphi$ in the $\theta$ dimension.

Consider the given image $f(x, y)$ with a square object not aligned at the center as illustrated in Figure 9(a), its Radon transform, as discussed before, shows a sinusoidal shift in $\rho$ as shown in Figure 9(b). Suppose that the square object is rotated by $45^{\circ}$ with respect to its centroid as illustrated in Figure 9(c). The Radon transform shown in in Figure 9(d) reflects that the object rotation of $45^{\circ}$ is a shift of Radon transform coefficients by $45^{\circ}$ in the $\theta$ axis. The two peaks located in $45^{\circ}$ and $135^{\circ}$ angle in Figure $9(\mathrm{~b})$ are shifted downward to $0^{\circ}$ and $90^{\circ}$ angle, respectively, in $\theta$, as shown in Figure 9(d). Consequently, the rotation of an off-center object produces a shift in $\theta$ and also sinusoidal shift in $\rho$ axis. Since the cardinal multiwavelet transform of $\rho$ axis is almost shift invariant, the CMRT of $f(x, y)$ is approximately shift invariant and approximately orientation invariant except with a shift in $\theta$ variable. 
Figure 9(e) and Figure 9(f) are the single ridgelet coefficients of Figure 9(a) and Figure 9(c). Rotation of the object made shifts of the Radon coefficients both in $\theta$ and $\rho$ axes, thus the amplitude of the high frequency coefficients of the ridgelet transform changes drastically. On the other hand, magnitude of the high frequency coefficients of the CMRT shown in Figure $9(\mathrm{~g})$ and Figure 9(h) is nearly invariant except with a shift in $\theta$ designating the rotation. 


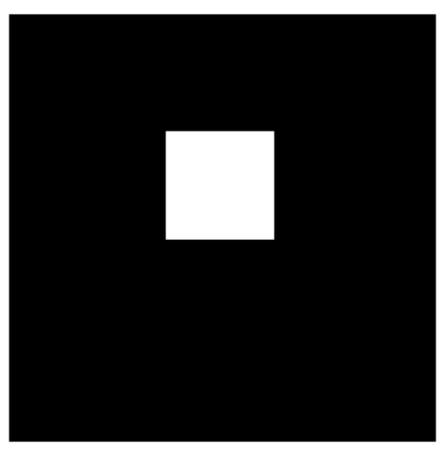

(a)

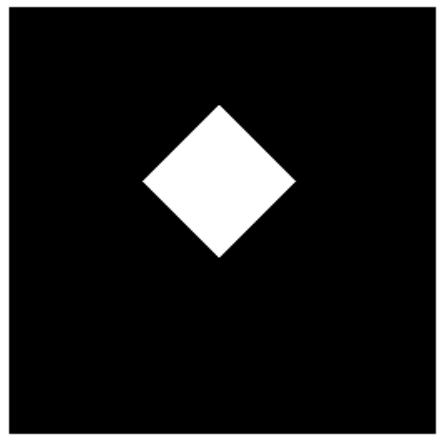

(c)

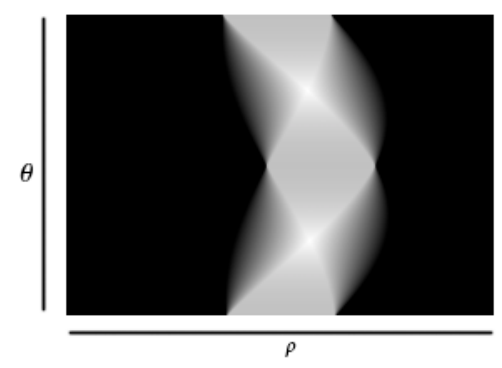

(b)

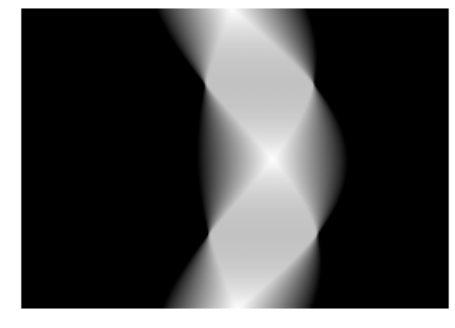

(d) 


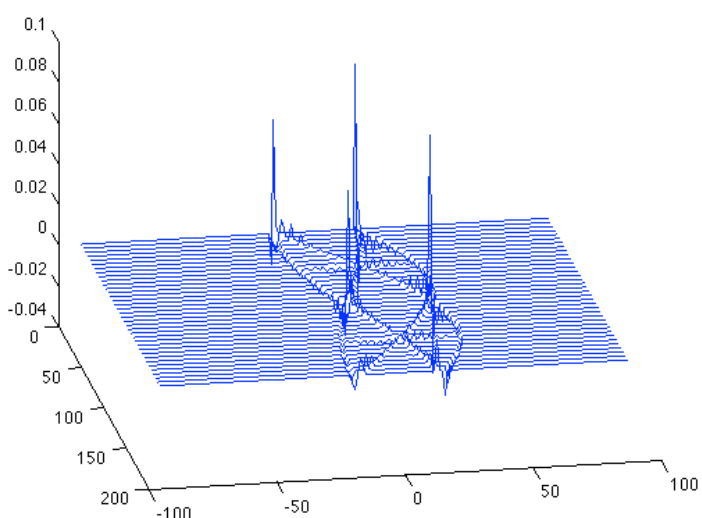

(e)

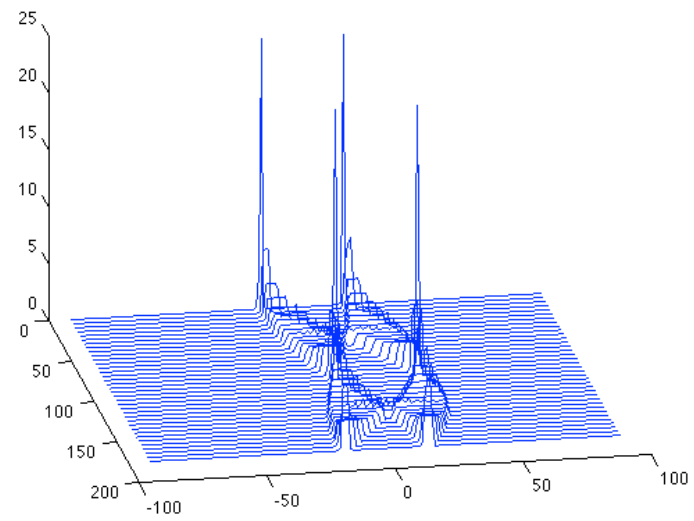

(g)

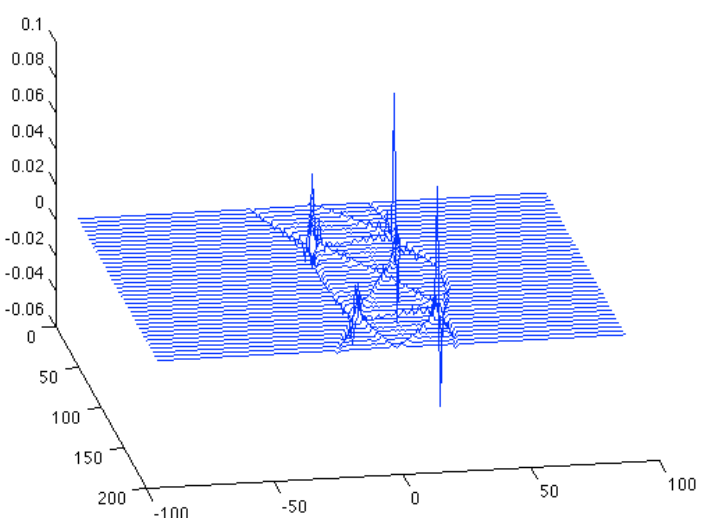

(f)

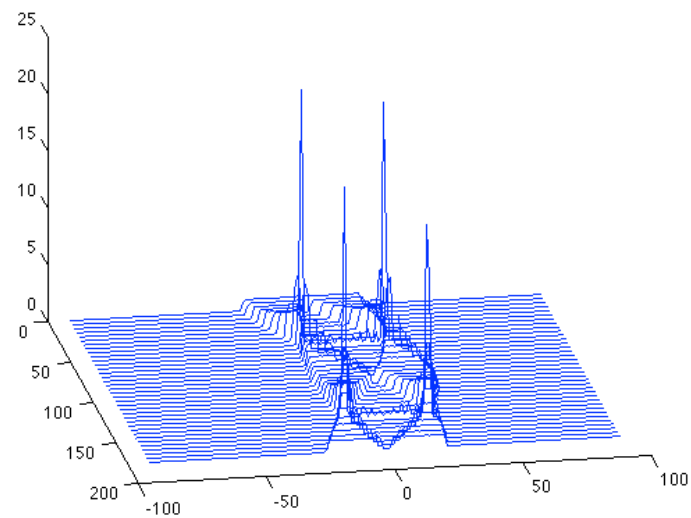

(h)

Figure 9 Sample 2-D images with square object and its Radon transform coefficients, (a) object off from the center, (b) Radon coefficients of (a), (c) object rotated 45 degree, (d) Radon coefficients of (c), (e) high frequency coefficients of ridgelet transform of (a), (f) high frequency coefficients of ridgelet transform of (c), (g) magnitude of high frequency scale coefficients of the CMRT of (a), and (h) magnitude of high frequency scale coefficients of the CMRT of (c). 


\subsubsection{Advantages of CMRT-based texture analysis}

One advantage of the cardinal multiridgelet transform (CMRT) inherited from the Radon transform is its robustness to noise. Since the Radon transform is composed of line integrals of an image, the effect of any additive zero-mean Gaussian noise in the image will be essentially cancelled out, hence the CMRT of the image is nearly noise-free. It may result in a more faithful texture representation from the CMRT coefficients.

The advantages inherited from the cardinal orthogonal multiwavelet transform are its cardinality, orthogonality and compact support. Cardinality is an important property because, for a cardinal scaling function as the sampling function in the scaling space, there is no aliasing error in the cardinal wavelet transform, therefore we can expect to obtain a more effective ridgelet decomposition for extraction of image texture features. Orthogonality along with cardinality in the dual-tree provides a balanced multiwavelet system. The cardinal balanced multiwavelet has the shortest support, beside the GHM multiwavelet, which is shorter than that of the dual-tree complex wavelets.

The two approximate invariance properties of the CMRT discussed in the earlier sections are important for image texture analysis, because they enable to extract invariant features regardless of the position and orientation of the region of interest. The key aspect is the shift invariance of the transform within the passband of each signal component. An object rotation is manifested by a shift in the theta-axis along with a sinusoidal shift in the radial axis, so the feature description will remain unchanged except with a simple rotation. These properties are particularly important in medical image texture classification. 


\subsection{APPLICATIONS TO TEXTURE ANALYSIS}

Textures, a function of spatial variations in pixel intensity, provide important characteristics for object recognition and categorization. Numerous techniques for automatic texture analysis have been developed during the past half-century. Wavelet-based texture recognition and classification have shown many successful applications in recent years. Texture description using directional wavelets is an important development. This includes ridgelets, curvelets, and contourlets. The applications of the cardinal multiridgelet transform (CMRT) to texture analysis has been explored in three studies, two of which are discussed in this chapter: texture database retrieval and LCD "mura" defect detection. The third study on prostate cancer tissue image classification will be presented in the next chapter.

\subsection{TEXTURE DATABASE RETRIEVAL}

Texture database retrieval using Gabor wavelet-based feature extraction has been reported by many studies [28-30], and some are referenced as a baseline performance of texture database retrieval tests [31][32]. Directional wavelets have also been applied such as complex wavelets [31], contourlets [32], and ridgelets [33]. In this study, we examine the application of CMRT for 
texture database retrieval performance, in comparison to the use of Gabor wavelet, ridgelet, and curvelet.

\subsubsection{Feature vector from the cardinal multiridgelet transform}

The order-2 CMRT was applied for 3-scale decomposition of images. At each scale, the CMRT coefficients were divided into subbands with respect to orientations. The low frequency coefficients (at the scale level 3) constitute one subband, the first high frequency region (at scale level 3) is divided into 3 orientations, the next higher frequency region (at scale level 2) is divided into 6 orientations, and the highest frequency region (at scale level 1) is divided into 9 orientations with respect to directions. Therefore, the total number of subbands is 19 .

Let $W$ be a matrix of transform coefficients. Its first subscript indicates transform method and channel, e.g., CMRT coefficients $W_{C M R T 0}$ are from the first channel and $W_{C M R T 1}$ from the second channel. The first channel matrix element $W_{C M R T 0, m n}$ has two indices $m$ and $n$, subscript $m$ indicates scale level where low number means a coarser scale and higher number means a finer scale, and subscript $n$ is an index of orientation. Since there are both low frequency and high frequency components at the lowest scale level, they are denoted by $m=1$ and $m=2$ respectively; so for the 3 -scale decomposition used in this study, $m \in[1, \cdots 4]$. For the low frequency coefficients at the coarsest scale, there is no orientation division, so there is only one subband denoted by $W_{C M R T 0,11}$ at scale index $m=1$. But at the finest scale $m=4$, there

are 9 divisions (subbands): $W_{C M R T 0,41} \sim W_{C M R T 0,49}$. Similar notations are used for the second channel, $W_{C M R T 1, m n}$. Coefficients from both channels are combined into the magnitude 
$W_{C M R T, m n}=\sqrt{\left|W_{C M R T O, m n}\right|^{2}+\left|W_{C M R T 1, m n}\right|^{2}}$. Figure 10 illustrates the subband allocation of CMRT coefficients.
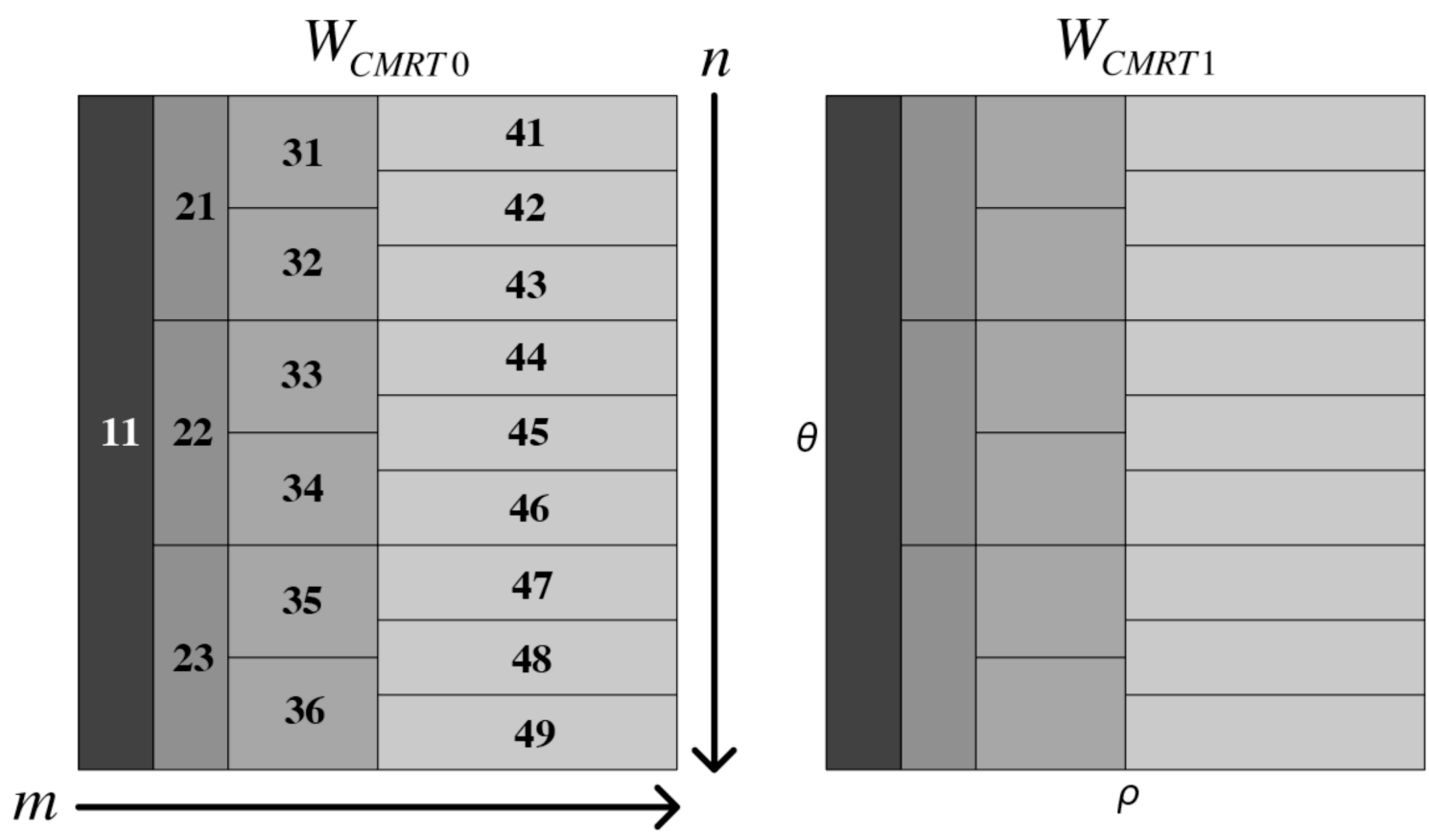

Figure 10 CMRT decomposition to 3-scale and subband allocation with respect to scale and orientation. 
Three statistical features were computed for each coefficient subband: mean $\mu_{m n}=\mu\left(W_{C M R T, m n}\right)$, standard deviation $\sigma_{m n}=\sigma\left(W_{C M R T, m n}\right)$, and entropy $s_{m n}=s\left(W_{C M R T, m n}\right)$. For a given texture image indexed by $k$ in the texture database, the feature vector extracted from the CMRT coefficients is given by

$$
\underline{F}_{C M R T}(k)=\left[\mu_{11}(k), \sigma_{11}(k), s_{11}(k), \cdots, \mu_{49}(k), \sigma_{49}(k), s_{49}(k)\right]^{T}
$$

where the number of elements of the feature vector $\underline{F}_{\text {CMRT }}(k)$ is $(1+3+6+9) \times 3=57$.

For Gabor wavelet method, we used four scales and six orientations, the dimension of its feature vector $\underline{F}_{G A B O R}(k)$ is $6 \times 4 \times 3=72$. For curvelet method, the dimension of $\underline{F}_{\text {CURVELET }}(k)$ is 54, and for ridgelet method, the dimension of $\underline{F}_{R T}(k)$ is 57 . 


\subsubsection{Similarity measure}

The normalized Euclidean distance metric was used as a similarity measure, which is given by

$$
d(\underline{F}(i), \underline{F}(j))=\sum_{m} \sum_{n} d_{m n}(i, j)
$$

where

$$
d_{m n}(i, j)=\left|\frac{\mu_{m n}(i)-\mu_{m n}(j)}{\alpha\left(\mu_{m n}\right)}\right|+\left|\frac{\sigma_{m n}(i)-\sigma_{m n}(j)}{\alpha\left(\sigma_{m n}\right)}\right|+\left|\frac{s_{m n}(i)-s_{m n}(j)}{\alpha\left(s_{m n}\right)}\right|
$$

where $m$ and $n$ are the indices of subband of transform coefficients with respect to their scale and orientation, and $\alpha\left(\mu_{m n}\right), \alpha\left(\sigma_{m n}\right)$, and $\alpha\left(s_{m n}\right)$ are the standard deviations of the respective features over the entire database.

\subsubsection{Texture database}

The texture image set used in this study is from Brodatz texture photographic album [34]. Size of each texture image is $640 \times 640$. The texture database used in the experiment consists of 25 different texture classes, they are formed by cropping sixteen $64 \times 64$ non-overlapping subimages from each original $640 \times 640$ images of each texture classes, thus the database consists of 400 different $64 \times 64$ images represent 25 different classes of data, 16 samples per class, which is a 
relatively large number of classes and a small number of examples for each class. Figure 11 shows images in the database.
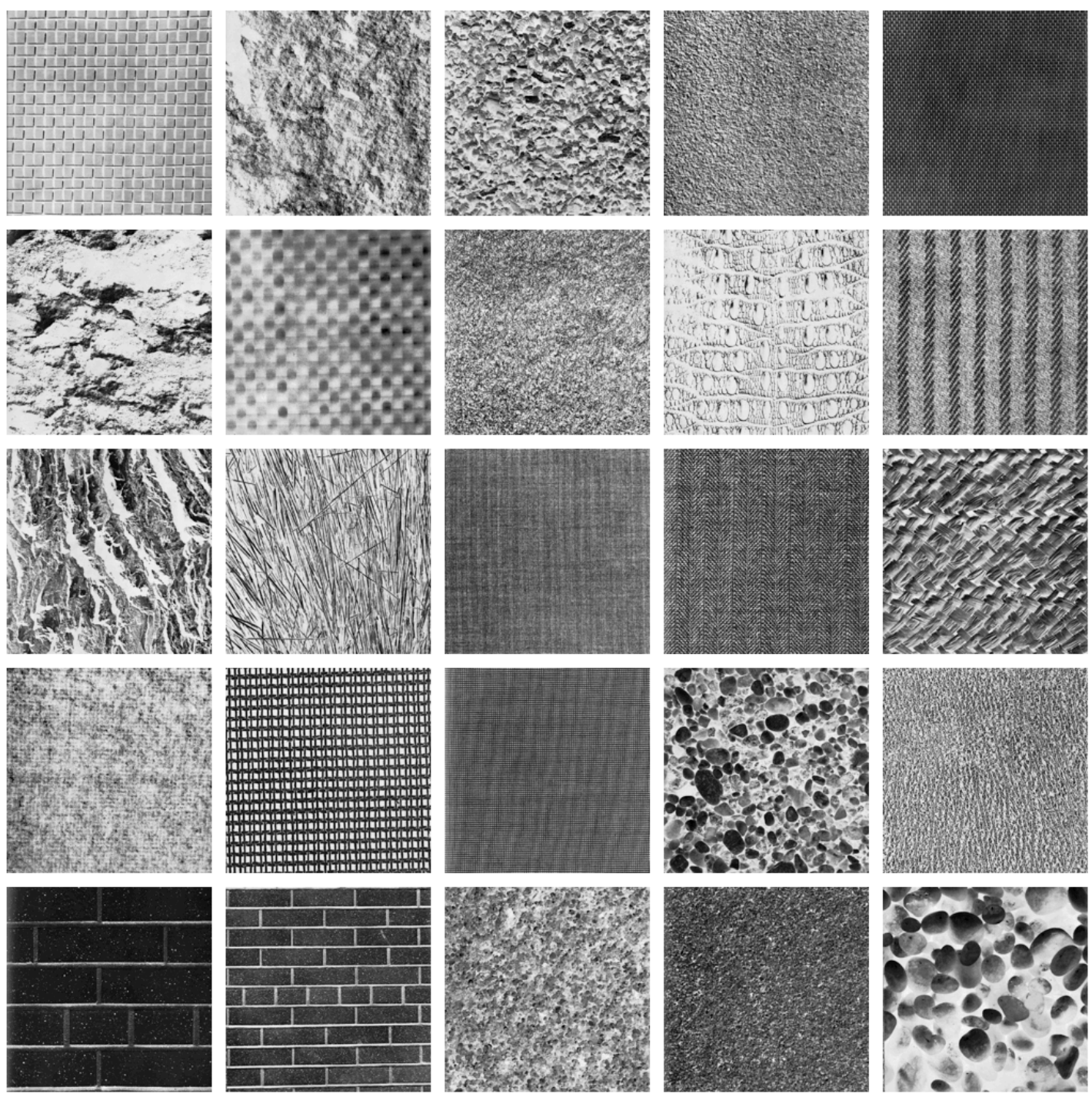

Figure 11 List of images consists of the texture database of the study. Each image is 640x640 pixel.

A query pattern is any one of the 400 patterns in the database. The pattern is then processed to obtain its feature vector. The feature vector distance $d(i, j)$, where $i$ denotes the query image and $j$ denotes any one element of the database, is computed. The distances are 
then sorted in increasing order, and the closest set of patterns is then retrieved. The performance is measured in terms of the average retrieval rate, which is defined as the average number of patterns belonging to the same class as the query image.

\subsubsection{Test result}

For each query image, $C$ closest matches are selected, and the number of those samples belonging to the same class of the query, except the query image itself, is counted; this number (less than or equal to 15) divided by 15 is defined as the retrieval rate. In the ideal case, all the closest 15 retrievals are from the same class. The performance of the entire class is obtained by averaging this rate over the 16 members. The overall average performance of the 25 classes results in the overall performance of the method.

Table 1 elaborates average retrieval rates of all texture classes using different methods at $C=15$. The CMRT method showed the best retrieval rate $75.22 \%$, followed by the curvelet method $74.75 \%$, and Gabor wavelet method $71.17 \%$, while single ridgelet method gave lowest rate $60.03 \%$. Figure 12 shows the overall performances for the case of $C$ varying from 15 to 100. The CMRT and curvelet method gave the similar overall performance, while the CMRT method were slightly better. 


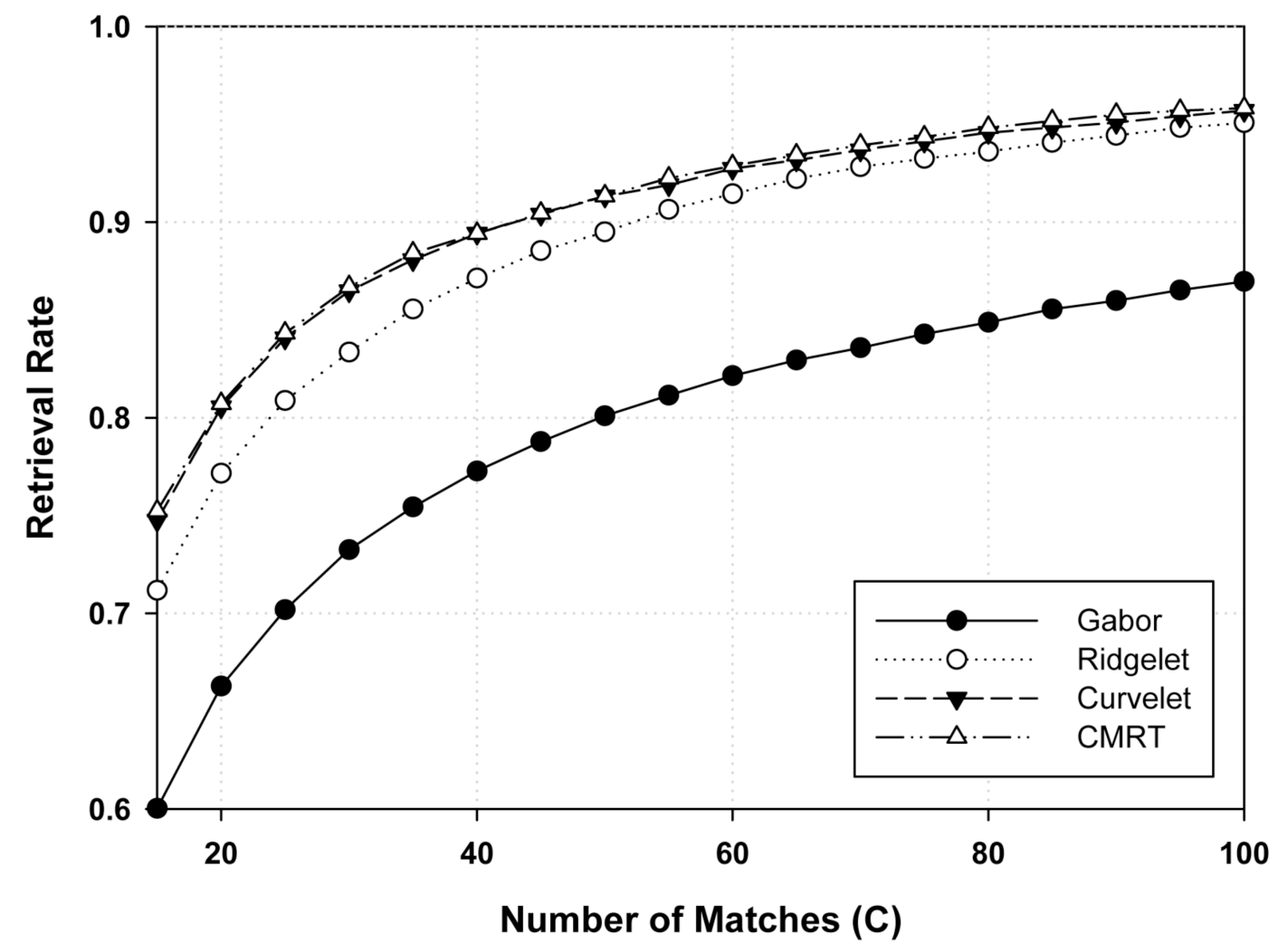

Figure 12 Average retrieval rate of texture database, the number of closest matches increased from 15 to 100 with step size 5 . 
Table 1 Average retrieval rate for the 25 texture images in database at $\mathrm{C}=15$.

\begin{tabular}{|c|c|c|c|c|}
\hline Images & Gabor & Ridgelet & Curvelet & CMRT \\
\hline D1 & $61.67 \%$ & $95.42 \%$ & $99.17 \%$ & $95.83 \%$ \\
\hline D2 & $60.00 \%$ & $55.00 \%$ & $57.92 \%$ & $50.00 \%$ \\
\hline D4 & $91.67 \%$ & $96.67 \%$ & $93.33 \%$ & $98.75 \%$ \\
\hline D5 & $45.83 \%$ & $37.92 \%$ & $40.00 \%$ & $52.08 \%$ \\
\hline D6 & $47.08 \%$ & $100.00 \%$ & $100.00 \%$ & $100.00 \%$ \\
\hline $\mathrm{D} 7$ & $14.58 \%$ & $35.00 \%$ & $35.00 \%$ & $37.08 \%$ \\
\hline D8 & $65.83 \%$ & $62.92 \%$ & $85.83 \%$ & $70.00 \%$ \\
\hline D9 & $84.17 \%$ & $81.67 \%$ & $86.25 \%$ & $85.83 \%$ \\
\hline D10 & $32.92 \%$ & $71.25 \%$ & $72.08 \%$ & $79.17 \%$ \\
\hline D11 & $76.67 \%$ & $82.92 \%$ & $77.92 \%$ & $79.58 \%$ \\
\hline D13 & $17.92 \%$ & $11.25 \%$ & $22.50 \%$ & $18.33 \%$ \\
\hline D15 & $68.75 \%$ & $66.25 \%$ & $64.58 \%$ & $65.83 \%$ \\
\hline D16 & $93.33 \%$ & $100.00 \%$ & $96.25 \%$ & $100.00 \%$ \\
\hline D17 & $65.42 \%$ & $94.17 \%$ & $98.75 \%$ & $95.83 \%$ \\
\hline D18 & $57.92 \%$ & $57.08 \%$ & $67.08 \%$ & $76.67 \%$ \\
\hline D19 & $65.00 \%$ & $65.42 \%$ & $68.75 \%$ & $68.75 \%$ \\
\hline D20 & $100.00 \%$ & $79.58 \%$ & $99.58 \%$ & $90.42 \%$ \\
\hline D21 & $62.08 \%$ & $100.00 \%$ & $100.00 \%$ & $100.00 \%$ \\
\hline D23 & $42.92 \%$ & $38.33 \%$ & $53.33 \%$ & $42.08 \%$ \\
\hline D24 & $71.67 \%$ & $73.33 \%$ & $84.17 \%$ & $89.58 \%$ \\
\hline D25 & $47.92 \%$ & $95.42 \%$ & $83.33 \%$ & $95.00 \%$ \\
\hline D26 & $63.75 \%$ & $66.25 \%$ & $71.67 \%$ & $79.58 \%$ \\
\hline D28 & $62.50 \%$ & $64.58 \%$ & $70.83 \%$ & $75.00 \%$ \\
\hline D29 & $65.83 \%$ & $90.42 \%$ & $94.58 \%$ & $94.17 \%$ \\
\hline D30 & $35.42 \%$ & $58.33 \%$ & $45.83 \%$ & $40.83 \%$ \\
\hline $\begin{array}{c}\text { Average at } \\
C=15\end{array}$ & $60.03 \%$ & $71.17 \%$ & $74.75 \%$ & $75.22 \%$ \\
\hline
\end{tabular}


An example shown in Figure 13 demonstrates the qualitative performance of the CMRT over other methods. In Figure 13(a) shows the retrieval of top 15 closest matches of the query image based upon Gabor wavelet method, Figure 13(b) is the retrieval based upon single ridgelet method, Figure 13(c) is the retrieval based on curvelet method, and Figure 13(d) is the retrieval based on CMRT method. In Figure 13(a) and Figure 13(b), it is seen that Gabor wavelet and single ridgelet methods retrieve perceptually similar images from the query image, however there are a number of samples from other classes of images. As is reported in Table 1, the retrieval rates of Gabor wavelet and single ridgelet methods for the query images of class "D24" are 71.67\% and 73.33\%. On the contrary, in Figure 13(c) and Figure 13(d), it is seen that curvelet and CMRT methods performing better than former two methods. Curvelet method misses only one sample, and CMRT method collects what is from the same class for all 15 closest matches. Overall, the retrieval rates of curvelet method and CMRT method for the query images of class “D24” are $84.17 \%$ and $89.58 \%$ respectively. 


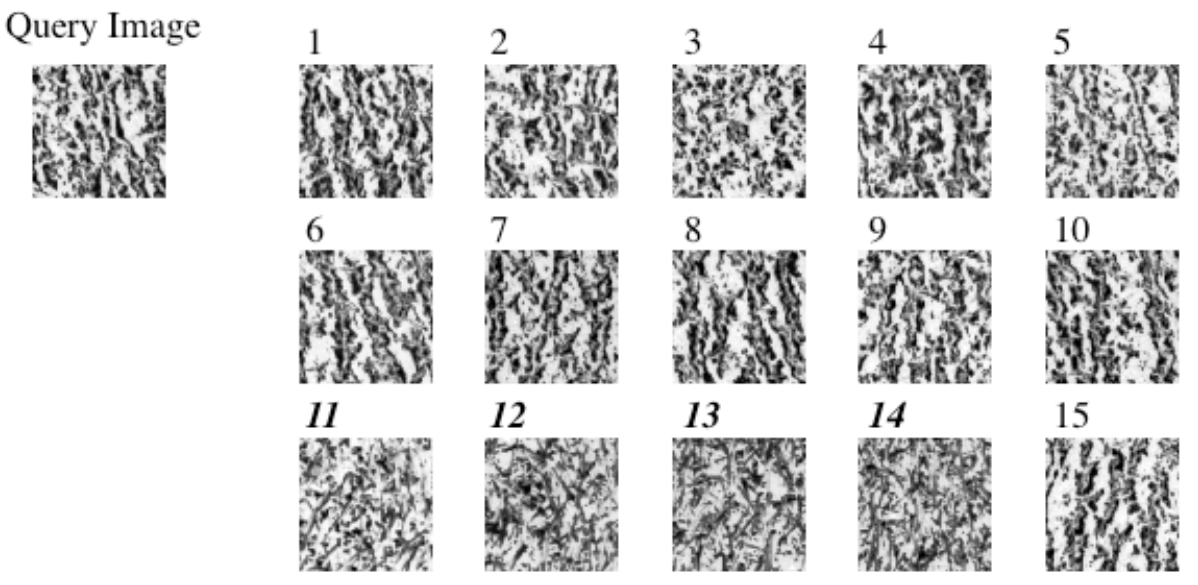

(a)

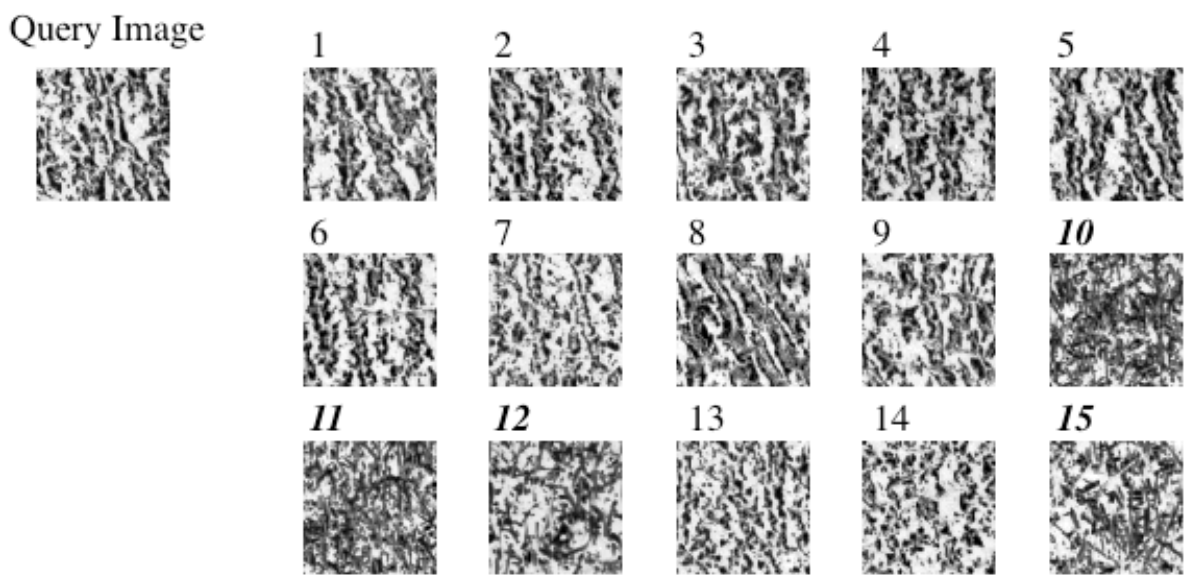

(b) 


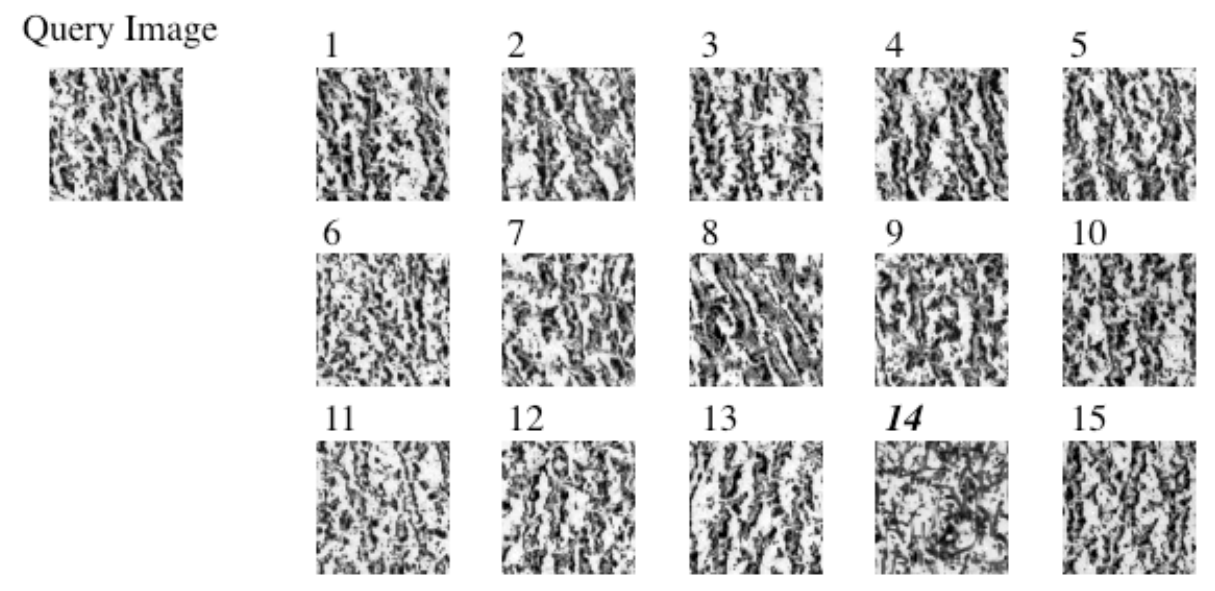

(c)

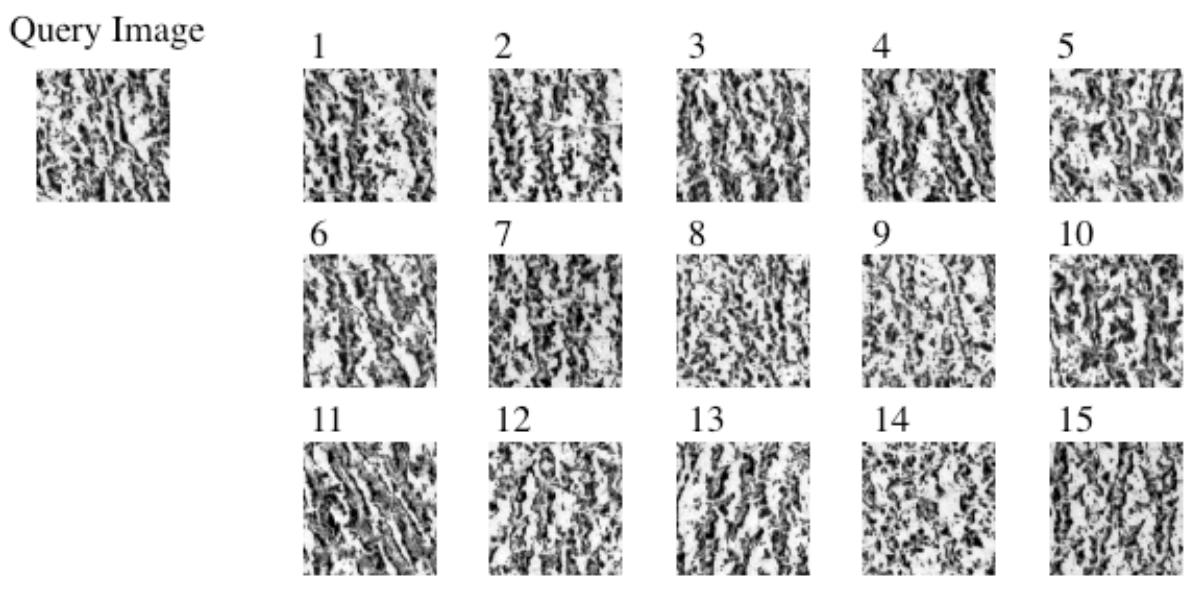

(d)

Figure 13 Example of closest 15 similar matched images for a given query image from the image class "D24", (a) using Gabor wavelets, (b) using single ridgelets, (c) using curvelets, and (d) using CMRT, bold italic rank index indicates where the corresponding subsample is belonging to another class. 


\subsection{LCD MURA DEFECT DETECTION}

The detection of "mura" defect on liquid crystal display (LCD) is essential to maintain the quality of the display. The term "mura" defect is derived from the Japanese word for blemish and is defined as irregular lightness dispersion without clear contour on the display. Such defects are resulted from improper cell thickness in a region, larger than a single pixel, typically appearing as low-contrast textural variations, and are visible when the display is driven to a constant signal level [35]. There are many reasons that cause mura such as uneven layer thickness, local non-uniformity in a chemical process, and local surface roughness. It depends on the human perception as to whether the mura is acceptable and how much the quality of the device degrades. However it is often more serious to the high resolution medical LCD display because a blob mura might be misclassified as a lesion. While LCD display manufacturing is highly automated, it is examined for defects detection mostly by human visual inspection, which is slow, costly, and becomes more difficult as panel size increases [36]. An automatic mura detection is beneficial to assure the uniform quality of the display product. This section discusses our study of applying the CMRT in feature extraction to detect blob mura defects.

\subsubsection{Linear support vector machine classifier}

The choice of feature classifier for the study is a linear Support Vector Machine (SVM) classifier [37]. Because of its capacity of prediction of unknown samples with a good degree of accuracy, SVM provides superior classification performance in higher dimensional feature space with relatively less training samples than other classifiers [38]. 
Training of a 2-class SVM is the task of finding separation hyperplane parameters

$(\underline{w}, b)$, which maximize the separation margin of the closest data samples from two classes if all the training samples are linearly separable. For an input feature vector $\underline{x}_{k}, k \in[1, \cdots K]$ where $K$ is the total number of training samples, the decision function is

$$
g\left(\underline{x}_{k}\right)=\underline{w} \cdot \underline{x}_{k}+b\left\{\begin{array}{cc}
\geq 0 & \text { for class 1 (abnormal) } \\
<0 & \text { for class 2 (normal) }
\end{array}\right.
$$

Even when the training samples are not $100 \%$ separable by a hyperplane in the feature space, slack variables can be introduced to train the best linear SVM for minimum training error. The Smooth SVM [39] software was used in this study.

\subsubsection{Simulation of artificial mura defects}

Since mura defects are vague and complex, it is difficult to establish an accurate mathematical model [40]. Typically, a blob mura defect appears somewhat circularly shaped with lowcontrast, and having no distinctive boundaries, therefore it is often modeled as an isotropic Gaussian shaped function with various ranges of radii and intensity levels [41], typically larger than a single pixel.

In this study, the CMRT method was applied in feature extraction of samples digitized from the Dome C5i active matrix LCD medical display by Planar, Inc. To capture normal display surface, set $50 \%$ intensity to the display, and capture it with a digital camera. The LCD 
has $165 \mu \mathrm{m}$ spatial resolutions for each pixel. In the captured image, $5 \times 5$ image pixels are rendering one LCD pixel.

Consider that in the medical display for mammographic studies, each microcalcification has an average diameter from $0.3 \mathrm{~mm}$ to about $0.7 \mathrm{~mm}$ [42]. A typical resolution of the digitized mammograms is $50 \mu \mathrm{m}$ per pixel (pixel dimension of a slide: $3540 \mathrm{x} 4740$ ) or $100 \mu \mathrm{m}$ per pixel (pixel dimension of a slide: 1770x2370) [43]. The 5 megapixel display (pixel dimension of display: 2048x2560) shows a slide at full-screen with resolution around 90 100 $\mu \mathrm{m}$. Therefore, subsampling LCD images by $256 \times 256$ pixels is adequate to measure the blob mura defects which can affect detection and classification of digital mammography.

An isotropic Gaussian function was placed in the center of a subsampled image to form a simulated abnormal case. Various intensity (1.5 15 pixel intensity) and size (8 24 pixels) of the blob mura were supplied for training data. Figure 14 demonstrates two examples of the simulated mura defects.

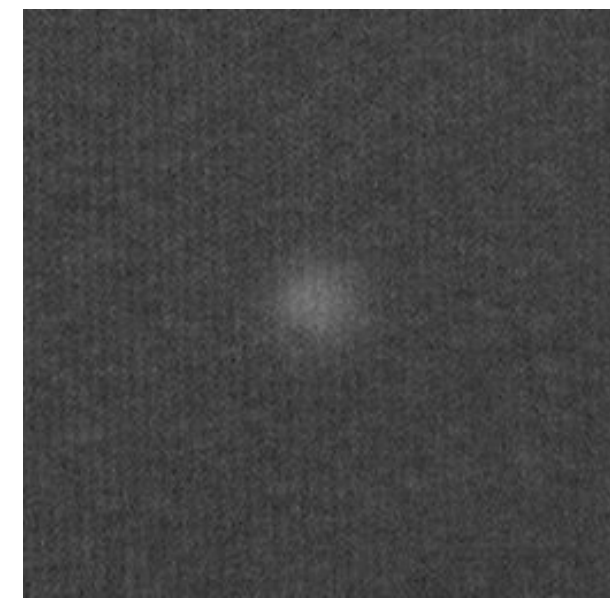

(a)

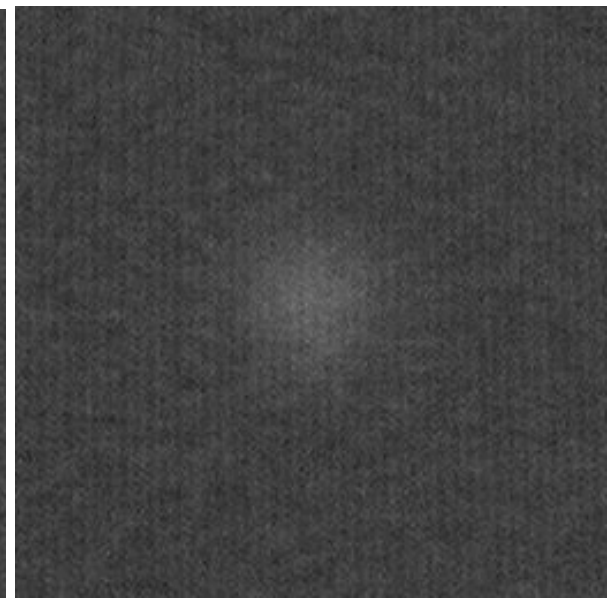

(b)

Figure 14 An example of simulated mura defects. An isotropic Gaussian function is placed at the center of the 256x256 sampled block of LCD display image displaying 50\% grayscale level: (a) mura with smaller radius and more intensity, and (b) mura with larger radius and less intensity. 


\subsubsection{Feature extraction in CMRT domain}

Mura defects are typically round shaped and omnidirectional. For classifying mura blobs, sensing where it is directed is not essential, but identifying circular objects superimposed to an even surface is required. Therefore in this study, CMRT coefficients are not partitioned directionally but all directions are considered in one subband.

Three-scale CMRT decomposition was taken for each 256x256 subimages. The 2channel CMRT coefficients were merged into a complex matrix where channel 1 coefficients are considered as the real part and channel 2 coefficients are considered as the imaginary part. Figure 15 shows how CMRT coefficients were partitioned. The magnitude of the merged coefficients was calculated by $W_{s}=\sqrt{\left|W_{0, s}\right|^{2}+\left|W_{1, s}\right|^{2}}$ where $s \in[0,1,2,3]$ is the scale index, and at the coarsest scale level the low frequency component is denoted by $s=0$ and high frequency component is denoted by $s=1$.
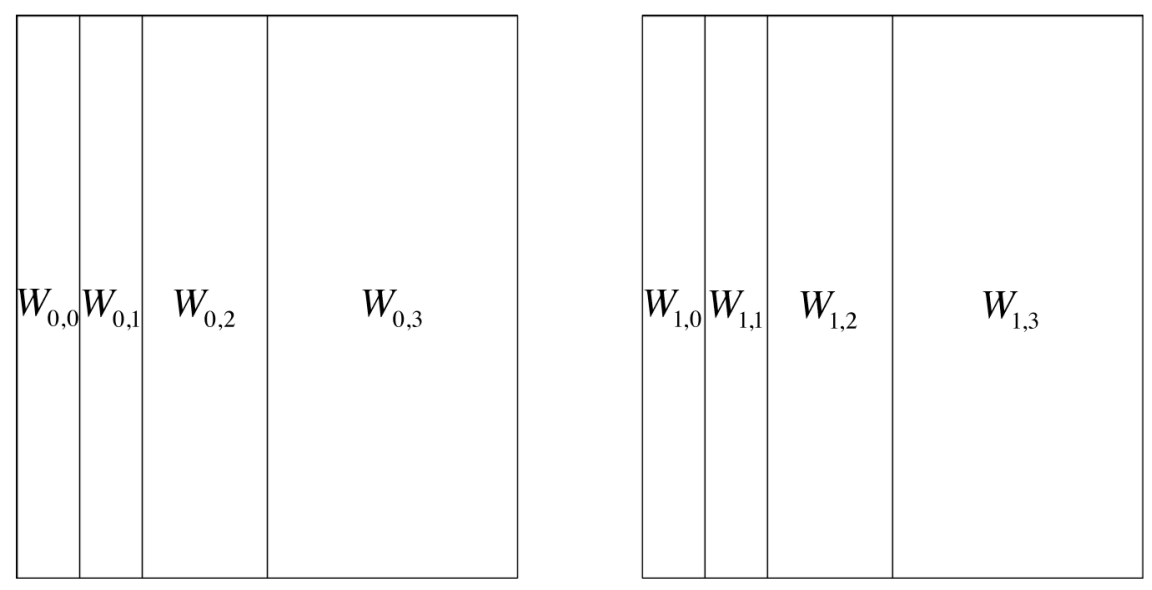

Figure 15 CMRT coefficients 3-scale decomposed, merged all directions. 
For each scale $s$, mean $\mu_{s}=\mu\left(W_{s}\right)$, variance $\sigma_{s}^{2}=\sigma^{2}\left(W_{s}\right)$, and entropy $s_{s}=s\left(W_{s}\right)$ features were calculated, and a feature vector $\underline{x}=\left[\mu_{0}, \sigma_{0}^{2}, s_{0}, \cdots, \mu_{3}, \sigma_{3}^{2}, s_{3}\right]$ was formed by features from all scales. Therefore the number of elements of the feature vector is 12 .

For comparisons, classifiers trained with features extracted by single ridgelet, Gabor wavelet, and curvelet methods were also studied. The 3-scale decomposed ridgelet coefficients were not partitioned directionally, but mean, variance, and entropy features were calculated for each subband. Therefore the number of ridgelet-based features extracted from a $256 \times 256$ subimage was also 12 .

Gabor wavelet in this study was utilized with 4 scales and 6 orientations. For each scale, mean, variance, and entropy features were calculated for each orientation, and then the average of each feature was taken for all orientations. Therefore the number of features extracted from 256x256 subimage using Gabor wavelet method was 12 .

The Curvelab Toolbox [44] was used for curvelet transform. The three-scale curvelet decomposition was taken. At each scale, mean, variance, and entropy features were calculated for each orientation, and each feature was averaged over for all direction. Therefore the number of features using curvelet method was 12.

\subsubsection{Classifier training and validation}

Training set was composed of 240 normal images and 240 simulated defective LCD images, and validation set was composed of 60 normal images and 60 simulated abnormal images. Extract features with the CMRT method (as well as with other methods mentioned above for comparative study), train a linear SVM with the training set of feature vectors and measure 
training accuracy with ROC curve and AUC measure if the training samples are not $100 \%$ linearly separable, and test the SVM classifier with the validation set and measure accuracy with ROC curve and AUC measure. As it turned out, the training data were not linearly separable under each feature extraction method, an optimum classifier was trained by introducing slack variables in the quadratic optimization process. The number of support vectors for CMRT based method was 41, Gabor based method was 22, ridgelet based method was 28 , and curvelet based method was 33 .

Figure 16(a) demonstrates the performance of each method with ROC curves using the training set. The first row in Table 2 is their comparison with area under ROC curve (AUC) measure along with their $95 \%$ confidence intervals, determined by using the JAFROC analysis software [45]. 


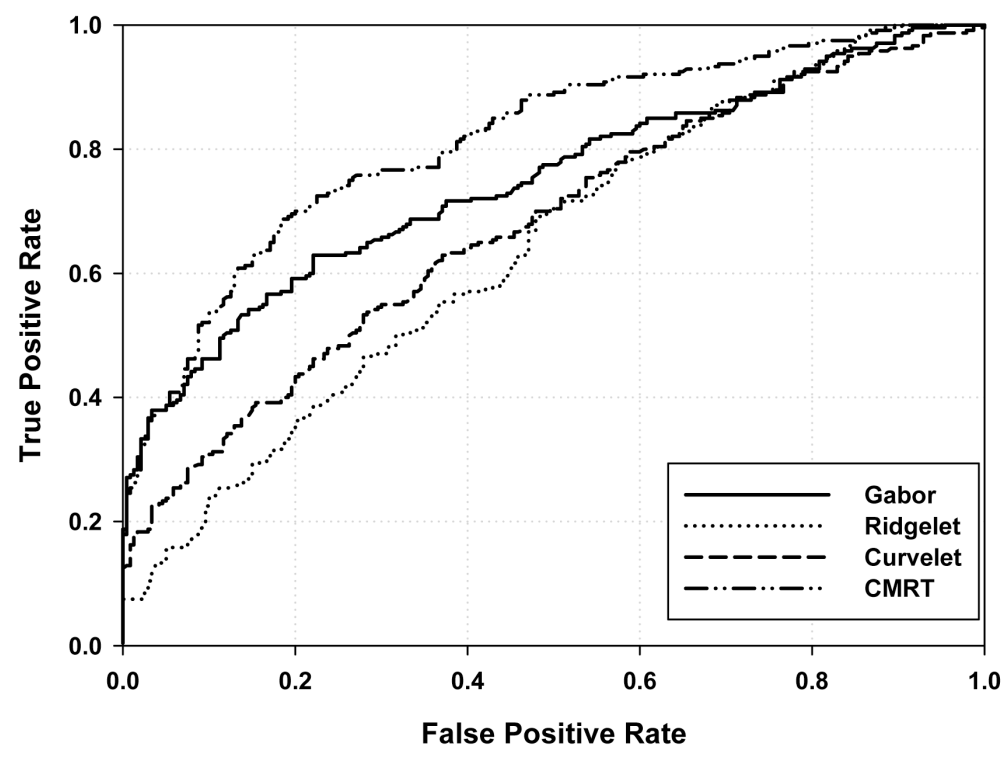

(a)

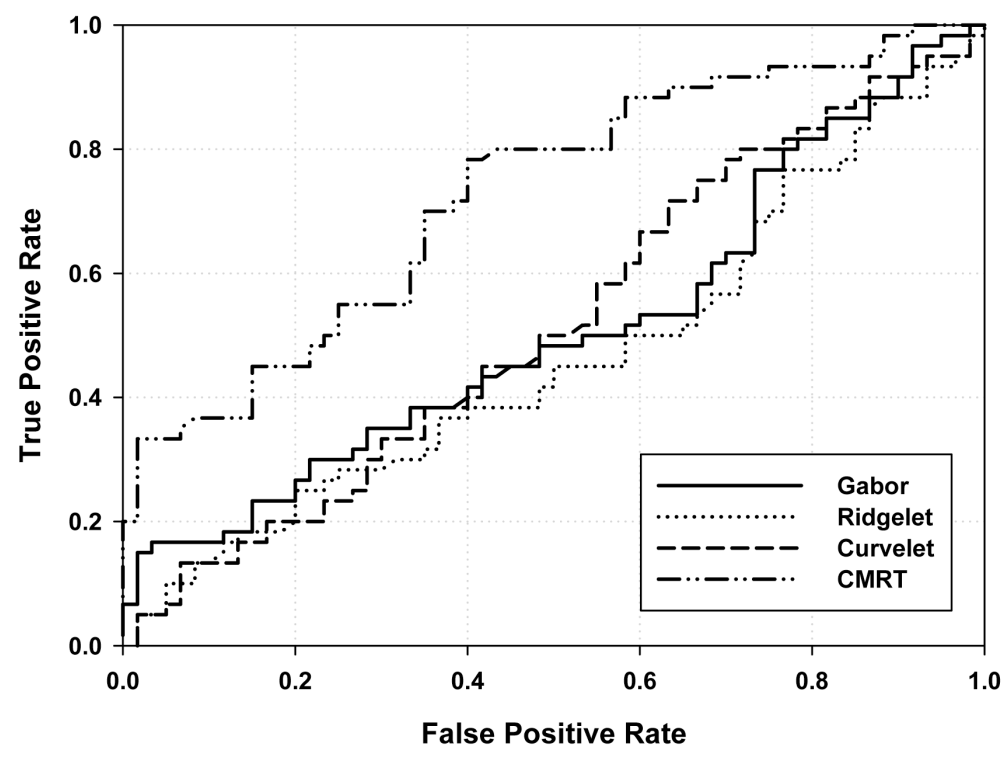

(b)

Figure 16 ROC curves of the mura defect classifiers based on (a) training sets and (b) validation sets. 
Table 2 Comparison of figures of merit in AUC of four methods, 95\% confidence intervals in parenthesis.

\begin{tabular}{|c|c|c|c|c|}
\hline & Gabor & Ridgelet & Curvelet & CMRT \\
\hline \multirow{2}{*}{ Training } & 0.7476 & 0.6414 & 0.6751 & 0.8142 \\
& $(0.7039,0.7914)$ & $(0.5923,0.6904)$ & $(0.6275,0.7227)$ & $(0.7765,0.8520)$ \\
\hline \multirow{2}{*}{ Validation } & 0.5094 & 0.4642 & 0.5183 & 0.7261 \\
& $(0.4028,0.6161)$ & $(0.3580,0.5703)$ & $(0.4125,0.6242)$ & $(0.6350,0.8172)$ \\
\hline
\end{tabular}

With the training set of the single ridgelet-based features, the trained linear SVM showed the least performance of $\mathrm{AUC}=0.6414$. The trained linear SVM with the curveletbased features also did not perform well. The AUCs of these two methods were each within 95\% confidence interval of the other. However, the linear SVM trained with the CMRT-based features showed the significant performance. Its AUC is higher than AUC's of other three methods, and was above their confidence intervals.

Superior performance of the CMRT-based method is also supported by the results obtained with the validation set. Figure 16(b) demonstrated that CMRT method outperformed the other three methods. The second row in Table 2 is the list of AUC's of all methods along with their $95 \%$ confidence intervals. Based upon these results, there is no doubt that CMRTbased features are significantly more effective than the other three methods in discriminating mura defects from the normal display.

\subsubsection{Mura defect detection}

Each trained linear SVM classifiers was then tested in detection study. A set of 40 normal and 40 abnormal images, each of 1280x960 pixels, were used in the detection study. In each abnormal image of simulating a defective display, two Gaussian blobs were superimposed to the 
image at arbitrary locations, as illustrated in Figure 17. For each image, 256x256 pixel subimages were taken, a feature vectors was extracted from the subimage, and it was classified by the trained linear SVM for detection of any mura block appearing in the image.

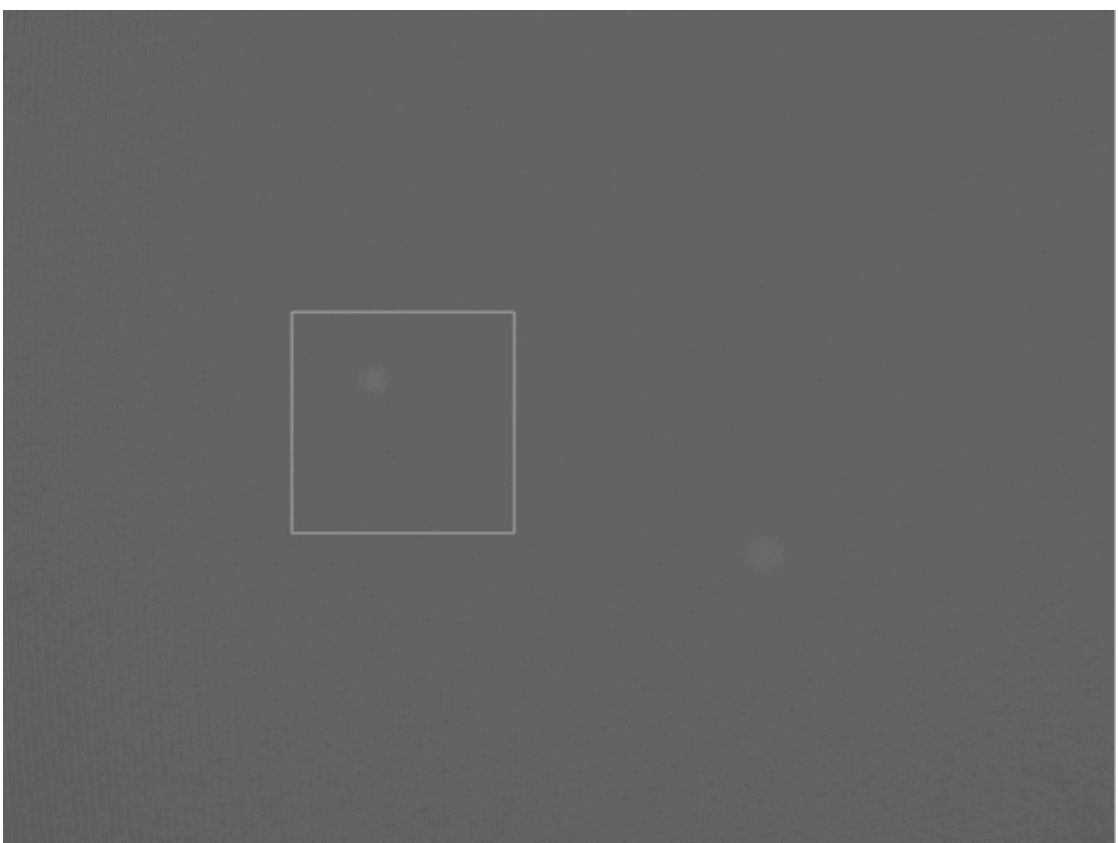

Figure 17 Sample of test image for Mura defect detection. Placed two simulated blobs with different radius and intensity superimposed to the screen. White box indicates where the $256 \times 256$ pixel subimage taken to the algorithm.

The DBM MRMC software [46] was utilized to quantify performances of the detection tests. Figure 18 gives the ROC curves of the detection tests of all four trained linear classifiers based on different feature extraction methods. The ROC fitting was performed by using PROPROC [47].

Table 3 is the list of AUC's and their 95\% confidence intervals. The AUC for CMRTbased method was 0.8208 , which outperforms the other three methods. It is significantly higher than that from Gabor wavelet-based and single ridgelet-based methods, and is slightly within 
the confidence interval of the AUC from the curvelet-based method. The AUC of the curveletbased method was 0.7218 , AUC from ridgelet-based method was 0.6934 , and that from the Gabor wavelet-based method was 0.6533 . They were all within the confidence intervals of each other.

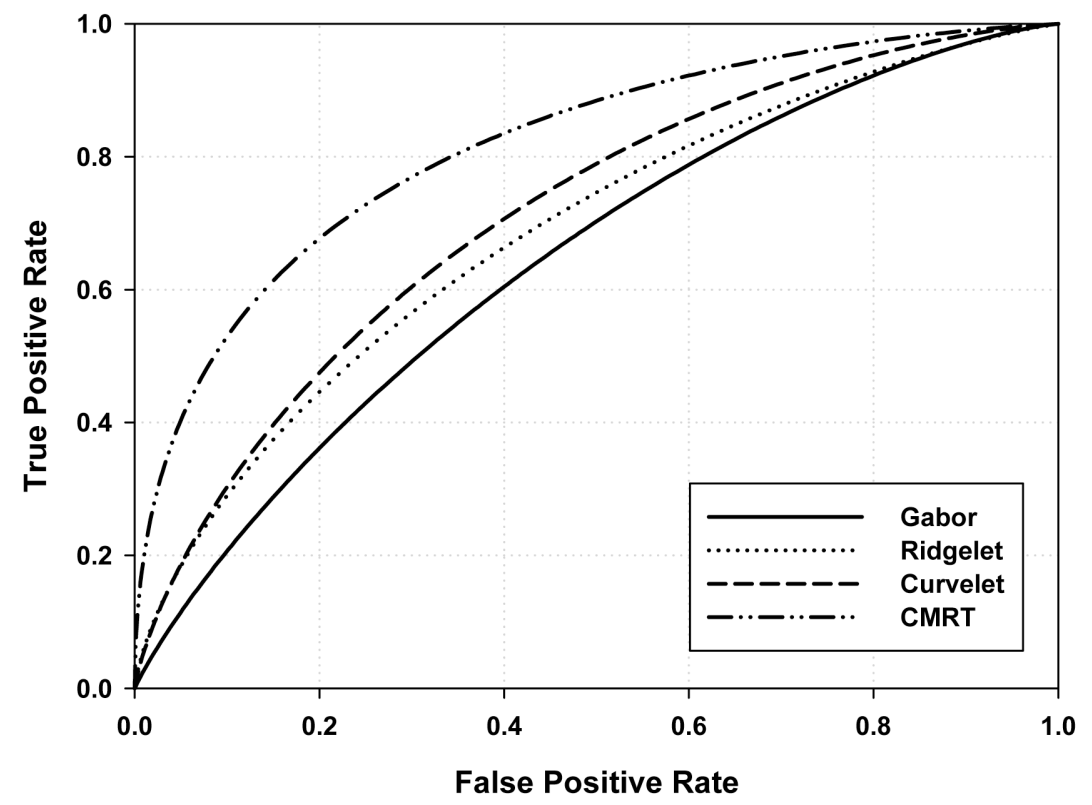

Figure 18 ROC curves of the detection test performance.

Table 3 Mura defect detection accuracy of all four methods, AUC and 95\% confidence intervals were determined by DBM MRMC software with PROPROC fit.

\begin{tabular}{|c|c|c|c|c|}
\hline & Gabor & Ridgelet & Curvelet & CMRT \\
\hline AUC & 0.6533 & 0.6934 & 0.7218 & 0.8208 \\
$(0.5400,0.7666)$ & $(0.5768,0.8099)$ & $(0.6193,0.8243)$ & $(0.7335,0.9081)$ \\
\hline
\end{tabular}




\subsection{SUMMARY}

In this chapter, the CMRT-based texture feature extraction method was applied to two experimental studies. The texture database retrieval study is a model example showing that the shift-invariant texture features are beneficial. Because the 16 patches were sampled from each image where the position of directional structures may be arbitrarily located, matching with shift-invariant texture features are necessary in order to provide a proper identification of the same class of images. This was demonstrated by the relatively high accuracy of the shiftinvariant methods such as our CMRT-based method and the curvelet-based method. The study on LCD mura defect detection, on the other hand, highlights the advantage of robustness to noise of the CMRT-based method which showed better performance than the curvelet-based method; because the curvelet transform is developed for sparse representation of smooth regions separated by smooth boundaries, while there is no clearly distinctive boundaries of the mura defects which are of extremely low contrast. 


\subsection{PROSTATE CANCER CLASSIFICATION}

Prostate cancer is one of the most frequently diagnosed cancers and ranks the second among the cancer deaths of men in the United States [48]. One of the most reliable detection methods of prostate cancer is the examination of prostate histological specimens under a microscope by pathologists. The histological grading of prostate cancer tissue is assigned according to the Gleason grading system [49][50]. It is based on microscopic tumor patterns assessed by pathologists while interpreting the biopsy specimen. The Gleason grading consists of five basic tissue patterns that reflect the degree of loss of normal glandular structure caused by the cancer. The grade, ranging from 1 to 5 , increases with the increasing level of malignancy, as illustrated in Figure 19. In essence, the Gleason grade characterizes the degree of resemblance of a tissue under examination to the normal tissue. Grade 1 designates a well-differentiated tissue having the highest degree of resemblance to the normal tissue, and grade 5 designates a very poorly differentiated tissue showing the drastic departure from the normal tissue pattern. The final Gleason score given to urologists is the sum of the most and the second most predominant grades seen in the prostate tissue. 


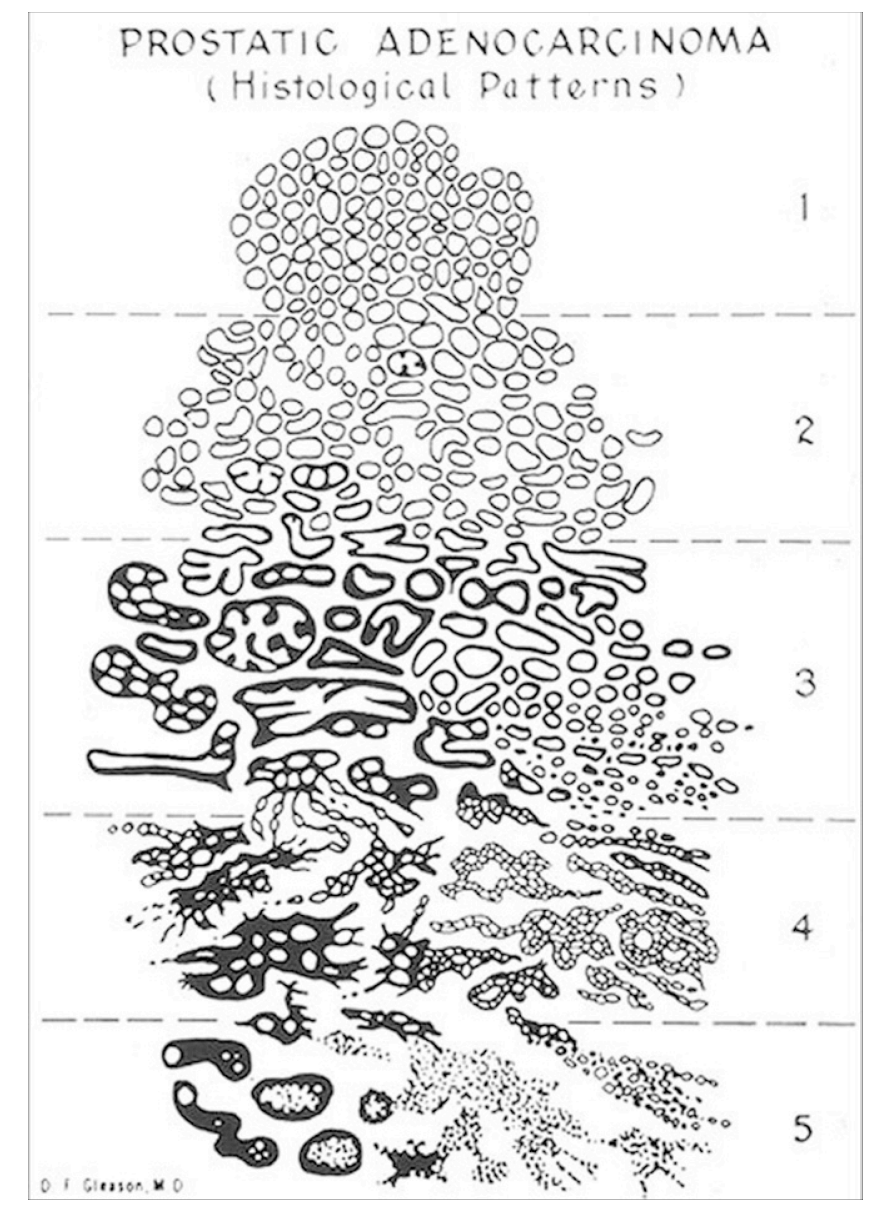

Figure 19 The Gleason grading system diagram by D.F. Gleason, M.D

Examples of prostatic adenocarcinoma for Gleason grade 2 to 5 are shown in Figure 20. In Figure 20(a), stroma, which is the fibromuscular tissue, clearly surrounds gland units, each of which is made of epithelial cells around a lumen; this retains to Gleason grade 2, where the glands are still round-shaped and are nearly uniformly distributed. Figure 20(b) is of Gleason grade 3, where glands are shown with an irregular arrangement due to the progress of cancer. Figure 20(c) illustrates Gleason grade 4; as cancer gets worse, epithelial cells replicate irregularly and tend to occupy lumen areas. Figure 20(d) shows Gleason grade 5, where lumens and stroma become virtually disappeared. 


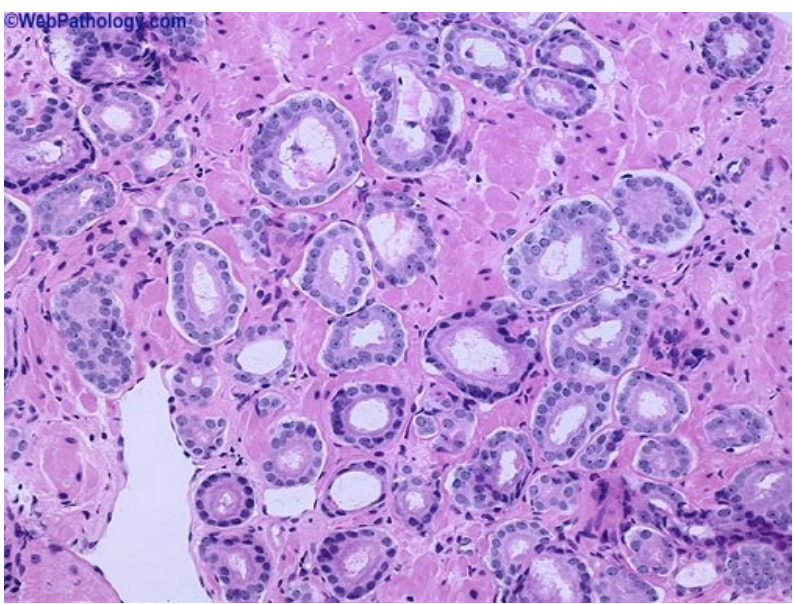

(a)

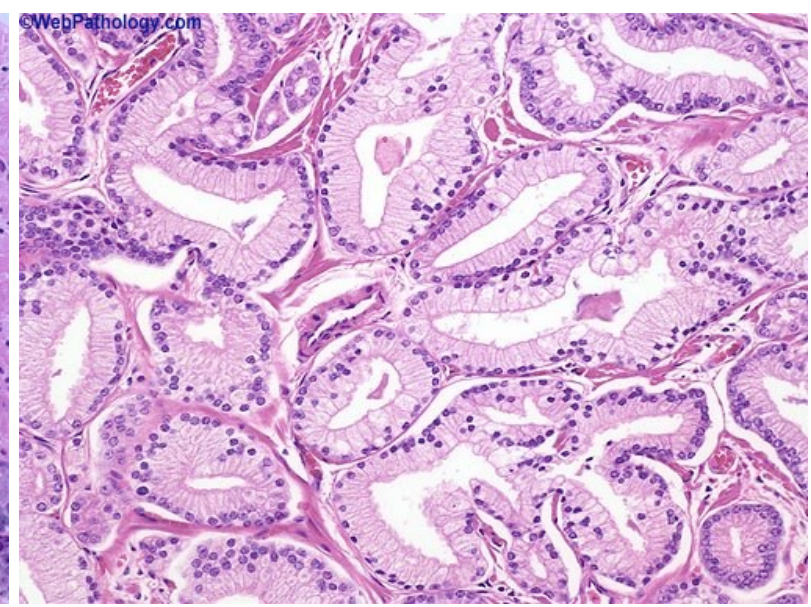

(b)

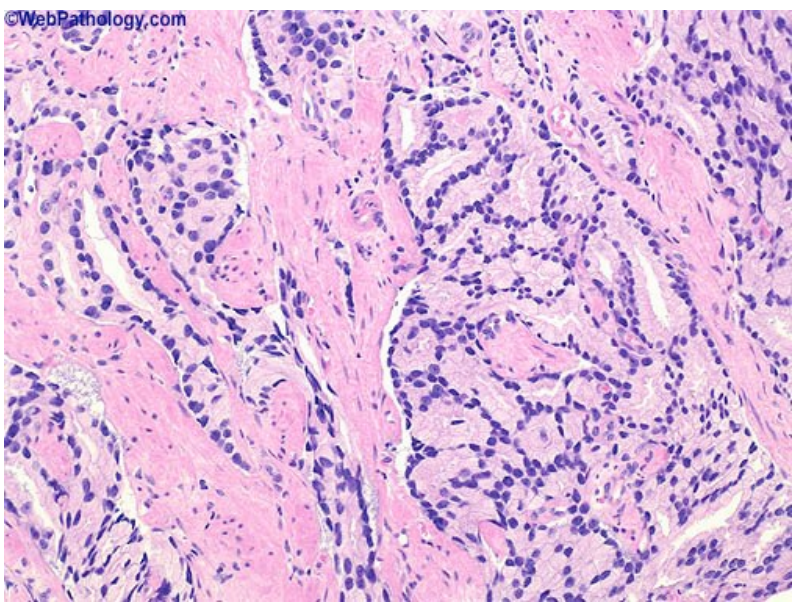

(c)

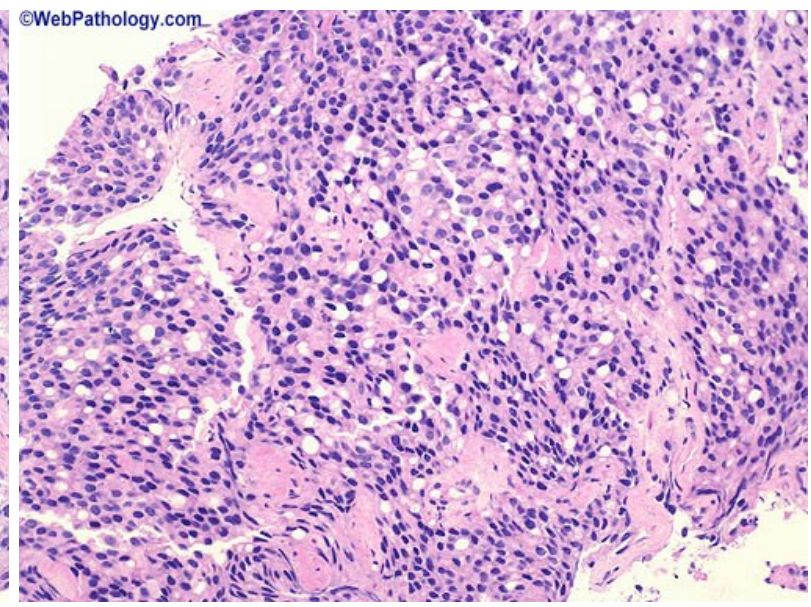

(d)

Figure 20 Examples of prostatic adenocarcinoma, (a) Gleason grade 2, (b) Gleason grade 3, (c) Gleason grade 4, and (d) Gleason grade 5, courtesy of WebPathology.com

Because human visual grading is very time-consuming and also subject to inter- and intra-observer variations, the development of machine vision techniques in aiding pathologists to analyze prostate tissue images and detect cancer in different stages has been in steady progress during the past decade. Applications of image texture analysis to computer-aided Gleason grading of prostate cancer tissues have been reported since 2003 [51-64]. Wavelet and 
multiwavelet transforms, fractal analysis, and texton forest / random tree have been utilized for texture feature extraction and classification in studies of automated Gleason grading [51-55][57$58][60-64]$.

As the malignancy of the prostate cancer is manifested by the loss of the normal glandular architecture (i.e. shape, size, and differentiation of the glands), it is appealing to apply the cardinal multiridgelet transform that has the excellent directional selectivity to represent the glandular architecture effectively. We thus propose to explore the application of the developed cardinal multiridgelet transform technique to extract tissue texture features for training a Gaussian-kernel support vector machine to aid the Gleason grading.

The biopsy Gleason score is the sum of the primary grade (representing the majority of tumor cells) and the secondary grade (assigned to the minority of the tumor cells), and is a number ranging from 2 to 10 . The higher the Gleason score, the more aggressive the cancer is likely to act and the worse the patient's prognosis will be. The primary Gleason grade has to be greater than $50 \%$ of the total tissue pattern seen. The secondary Gleason grade has to be less than $50 \%$, but at least $5 \%$ of the tissue patterns seen.

In reality, the primary Gleason grades 1,2 and 5 are rarely used in clinical situations due to their uncommonness [65], as a result, the interface between grade 3 and 4 (or Gleason score 6 and 7) is recognized as the boundary between the low-grade and the high-grade cancer, and it is there that most disagreements arise in second-opinion consultations. Attention to the Gleason grading and scoring has been focused on all patients with a Gleason score of 6 (Primary 3 and Secondary 3 (P3S3)), 7 (Primary 3 and Secondary 4 (P3S4), or Primary 4 and Secondary 3 (P4S3)), or 8 (Primary 4 and Secondary 4 (P4S4)). The presence of a secondary grade 4 is sufficient to label a cancer as of high grade overall [65]. Proper grading is a key to predict the 
patient's prognosis and to provide adequate prescription for treatment. The objective of the study here is to explore the use of the cardinal multiridgelet transform (CMRT) to aid the classification between Gleason grade 3 and grade 4 .

\subsection{METHODS}

The multiridgelet-based methodology for multiresolution texture feature extraction from prostate pathological images in described below. Non-linear support vector machines are developed for classification of Gleason grade 4 versus Gleason grade 3.

\subsubsection{Prostate cancer image database}

Prostate cancer images utilized in this study is the Tissue Microarray (TMA) 471 from the Pathology/Urology Departments of the Johns Hopkins University (under the courtesy of Dr. R. Veltri), that consists of 41 cases of patients with 2 needle biopsies per each patient, resulting in a total of 82 images of prostate specimens. Table 4 summarizes the number of images per each Gleason score. Note that P3S4 and P4S3 are separately listed for our study. Due to the limited quantity of sample in database, only a small number of features can be used in designing a classifier. 
Table 4 Number of samples per each Gleason score in TMA-471 database.

\begin{tabular}{|c|c|}
\hline Gleason score & Number of samples \\
\hline $6(\mathrm{P} 3 \mathrm{~S} 3)$ & 16 \\
\hline 7 (P3S4) & 18 \\
\hline 7 (P4S3) & 12 \\
\hline 8 (P4S4) & 32 \\
\hline 9 (P4S5) & 4 \\
\hline
\end{tabular}

\subsubsection{Feature extraction from cardinal multiridgelet packets}

As mentioned earlier, the merit of the cardinal multiridgelet transform (CMRT) is its directional selectivity, cardinality, approximate translation invariance and approximate rotation invariance properties. The ability to localize piecewise line (curvilinear) components is a key aspect in image texture classification problems. In our case of prostate cancer tissue image classification, it is important to localize directional components specifying structural textures of gland units and lumina, but it is not necessarily to know where they are actually directed. Therefore, in this study, CMRT coefficients are not partitioned in direction but pooled together all directions in each subband. On the other hand, more detailed decomposition in frequency is taken to distinguish the degree of disruption of the gland units; hence, multiwavelet packets are utilized in the study.

The CMRT packet decomposition of prostate cancer tissue image is developed as follows. The three-scale packet decomposition is taken in each direction of the Radon 
coefficients. As a result, there exist 8 packets for each of the two multiridgelet $(r=2)$ channels. Packet index is frequency ordered from 0 to 7,0 indicates the packet of the lowest frequency band, and 7 for the highest frequency band. As discussed in Chapter 2, the 2-channel CMRT coefficients are formed as complex coefficients where coefficients in channel 1 are regarded as the real part and in channel 2 as the imaginary part.

Let the CMRT packet coefficients from channel 1 be denoted as $W_{C M R P 0, p}$ and those from channel 2 as $W_{C M R P 1, p}$ where $p \in[0, \cdots, 7]$ denotes the packet index. The magnitude of the complex CMRT packet coefficient is given by

$$
W_{C M R P, p}=\sqrt{\left|W_{C M R P 0, p}\right|^{2}+\left|W_{C M R P 1, p}\right|^{2}}
$$

For each packet, variance $\sigma_{p}^{2}=\sigma^{2}\left(W_{C M R P, p}\right)$ and entropy $s_{p}=s\left(W_{C M R P, p}\right)$ are computed. Since $p \in[0, \cdots, 7]$ the maximum possible number of features is sixteen. Figure 21 show CMRT packet decomposition and index allocation of both channels. However, not all of the features can be considered in the classifier design due to the limited number of training samples available. Feature vector dimension must be reduced in order to train a reliable support vector machine yet preventing the occurrence of the over-fitting phenomena. 

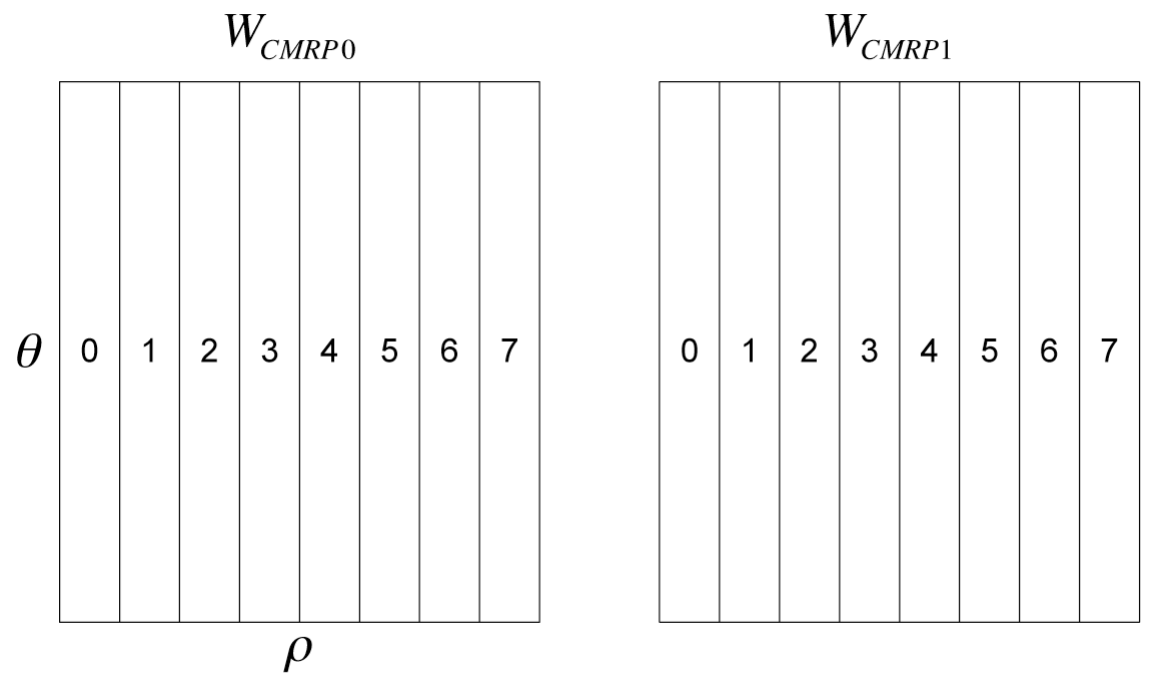

Figure 21 CMRT packet 3-scale decomposition.

For comparison purpose, similar experiments were performed with feature extraction based upon the curvelet approach. The Curvelab Toolbox software is used for the curvelet transform [44]. Three-scale decomposition was taken. For each scale, variance and entropy features were calculated from curvelet coefficients for each orientation, and then averaged over all direction for each feature. Thus, the number of features for curvelet method was 8 .

\subsubsection{Non-linear support vector machine classifier}

A preliminary study on feature sets from the tissue images showed that they are not linearly separable. So a non-linear classifier must be considered. Let the 2-class feature vectors $\left\{\underline{x}_{i} ; i=1,2, \cdots, N\right\}$, where $N$ is the number of training samples, be mapped into a higher 
dimensional space under a non-linear mapping $\underline{\phi}: \underline{x} \rightarrow \underline{y}$ such that $\left\{\underline{y}_{i}=\underline{\phi}\left(\underline{x}_{i}\right) ; i=1,2, \cdots, N\right\}$ become linearly separable, and let

$$
g(\underline{y})=\underline{w}^{T} \cdot \underline{y}+b=\underline{w}^{T} \cdot \underline{\phi}(\underline{x})+b
$$

be a discriminant function with $g(\underline{y})>0$ for $\underline{x}$ belongs to the class of Gleason grade 4 and $g(\underline{y})<0$ for $\underline{x}$ belongs to the class of Gleason grade 3.

To form a support vector machine (SVM) is to find the weight vector $\underline{w}$ and threshold weight $b$ so that the hyperplane $g(\underline{y})=\underline{w}^{\prime} \underline{y}+b=0$ in the $y$-space will have the maximum margin of separation for the given training data. Let $z_{i}$ designate the class index of the $i^{\text {th }}$ training pattern $\underline{y}_{i}\left(\right.$ or $\left.\underline{x}_{i}\right), z_{i}=1$ for patterns of Gleason grade 4 , and $z_{i}=-1$ for patterns of Gleason grade 3. Then

$$
z_{i}\left(\underline{w}^{\prime} \underline{y}_{i} b\right) \geq 1,(i=1,2, \cdots, N)
$$

and $\frac{1}{2}\|\underline{w}\|^{2}$ is to be minimized. 
It becomes an optimization problem to find $\underline{w}$ and $b$ for minimizing the criterion function $J(\underline{w}, b, \underline{\alpha})$ where $\left\{\alpha_{i} \geq 0\right\}$ are the associated Lagrange multipliers for the constraint $z_{i}\left(\underline{w}^{\prime} \underline{y}_{i}+b\right)-1 \geq 0$. Thus results in a non-linear SVM classifier as shown in Figure 22, where the non-linear discriminant function is given by

$$
g(\underline{y})=\sum_{i=1}^{N_{s}} \alpha_{i} z_{i} \underline{\phi}^{T}\left(\underline{x}_{i}\right) \underline{\phi}(\underline{x})+b=\sum_{i=1}^{N_{s}} \alpha_{i} z_{i} K\left(\underline{x}, \underline{x}_{i}\right)+b
$$

and $K\left(\underline{x}, \underline{x}_{i}\right)$ denotes a non-linear kernel

$$
K\left(\underline{x}, \underline{x}_{i}\right)=\left\langle\underline{\phi}(\underline{x}), \underline{\phi}\left(\underline{x}_{i}\right)\right\rangle=\underline{\phi}^{T}\left(\underline{x}_{i}\right) \underline{\phi}(\underline{x}),
$$

$N_{s}$ is the number of support vectors $\left(N_{s}<N\right)$ and support vectors are the training patterns that lie on the canonical surfaces $\underline{w}^{\prime} \underline{\phi}\left(\underline{x}_{i}\right)+b= \pm 1$ corresponding to $\alpha_{i}>0$. In this study, the Gaussian Radial Basis Function was used as the kernel function,

$$
K\left(\underline{x}, \underline{x}_{i}\right)=\exp \left(-\frac{\left\|\underline{x}-\underline{x}_{i}\right\|^{2}}{2 \sigma^{2}}\right) .
$$

The SVM and Kernel Methods Matlab toolbox was used for training our SVM classifiers [66]. 


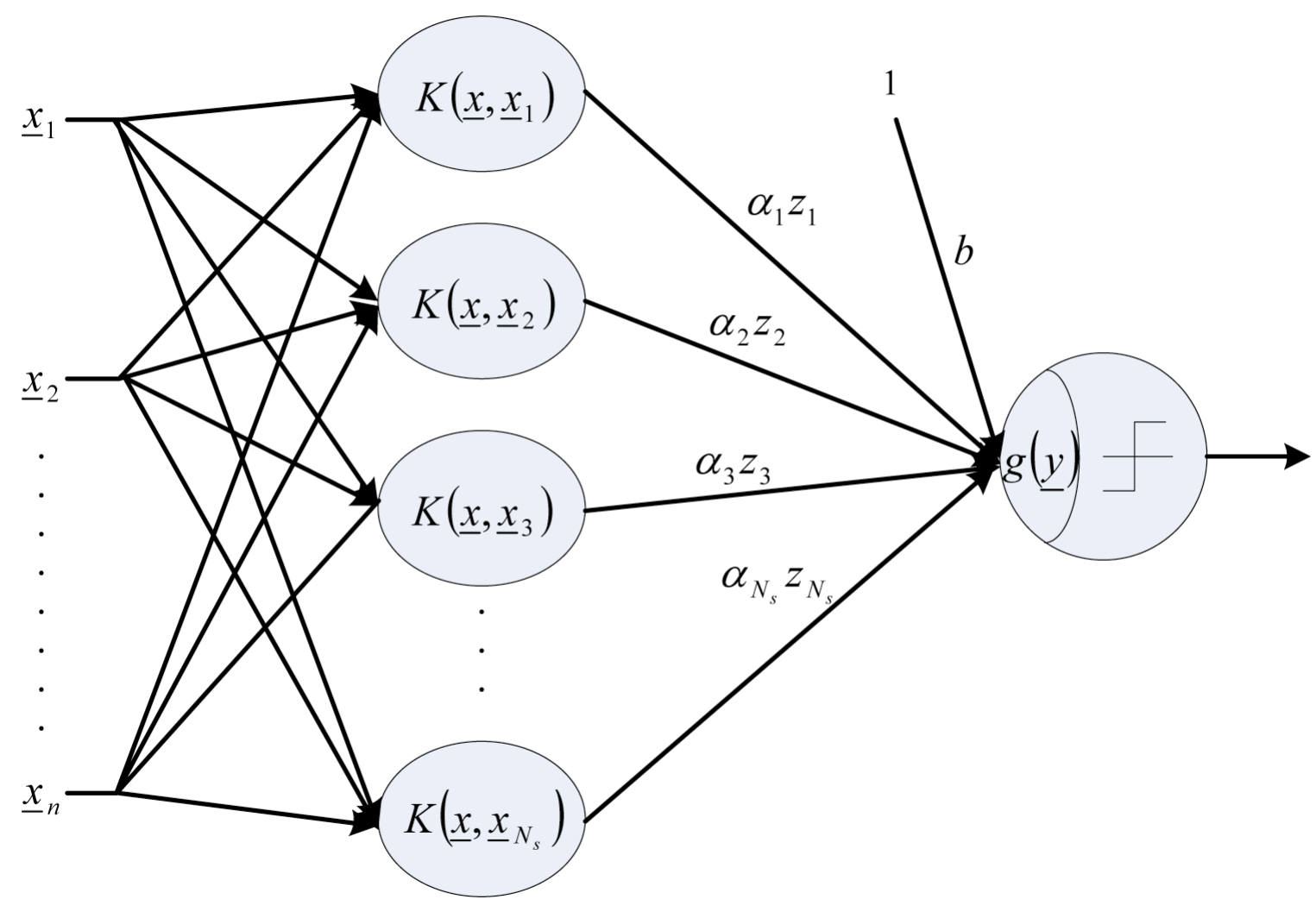

Figure 22 The non-linear support vector machine.

\subsubsection{Strategy in classifier design and cross-validation test}

Since we have a relatively small number of sample images and unbalanced datasets in the sense of unequal number of images per grade, we have drawn balanced datasets and developed three classifiers as described below. The leave-one-out cross-validation was used for the case of small number of training samples. While there are 32 samples of P4S4, there are only 16 P3S3 samples; to form a balanced training set for SVM, we took 16 samples from each class. Later, 
we added P3S4 samples and P4S3 samples into the pools for grade 3 and grade 4 respectively, to expand to 34 samples per class for training another classifier.

Cross validation is used to estimate the generalization capability of a trained classifier. Under cross-validation, the training data are divided into $k$ disjoint parts. Take one part out and train the classifier by $k-1$ parts and then test it by the taken-out part. This is repeated for all $k$ parts, and the test results are arranged. When $k$ is equal to the number of training samples, it is known as the leave-one-out (LOO) cross-validation. LOO cross-validation is suitable when the available training sample size is very limited, as it gives an indication how likely a small perturbation in the training data may affect a substantial change in the fitted model. In this study, the remaining P4S4 samples were utilized as a testing set. Even though it was a singleclass testing, nevertheless it added some information to the LOO cross-validation result.

The pixel resolution in TMA-471 database gives each image an array of 1650x1650 pixels, however, the tissue region on the each image is in a circular area. To extract features from each sample image, we took an array of $768 \times 768$ pixels from the center part of the image, which is sufficient to contain the prostate cancer cells architectural information. Then subdivided into 9 smaller blocks, each of 256x256 pixels, as shown in Figure 23. 


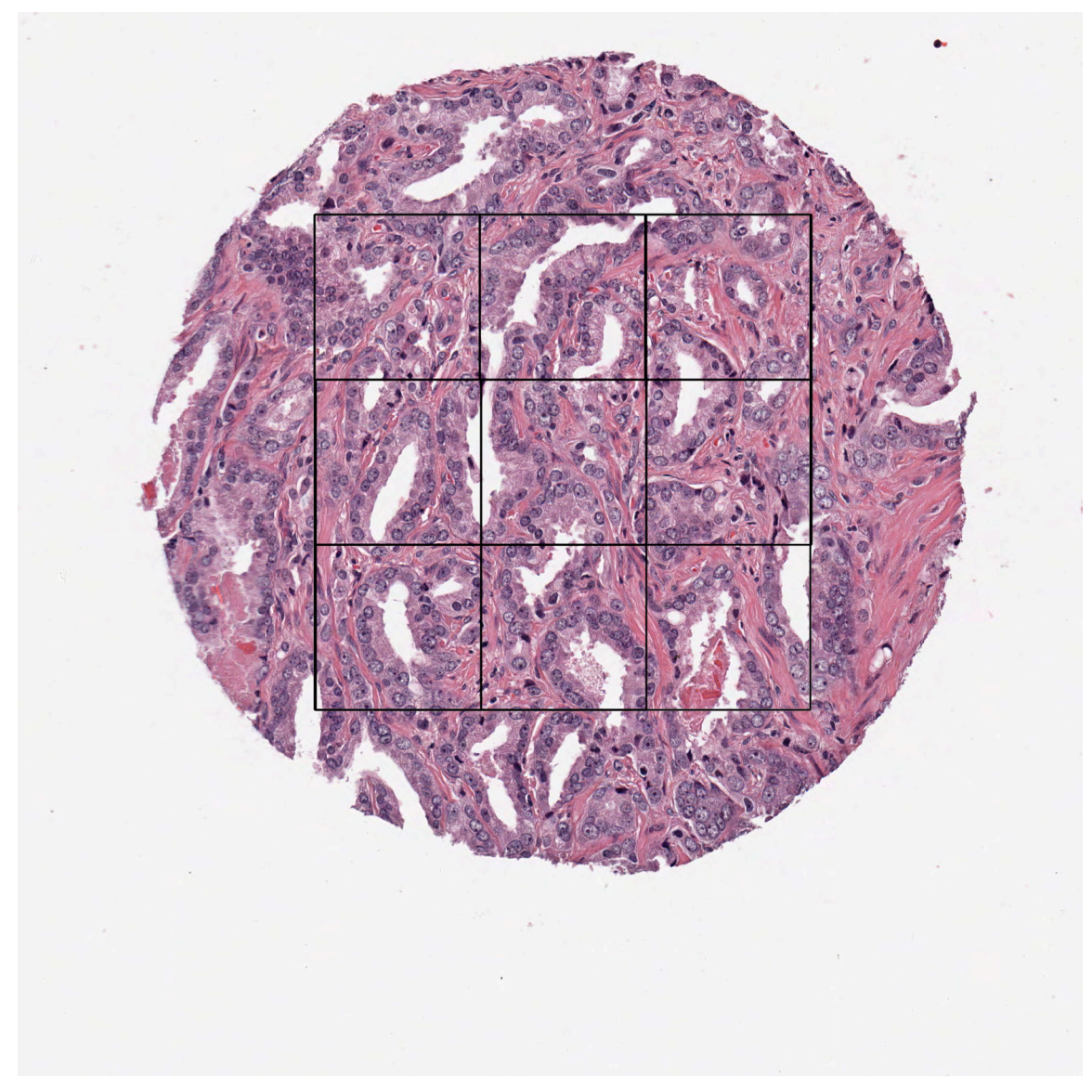

Figure 23 Subdividing prostate cancer images

Examine the output within each block, it appears to cover the fibroglandular structures of interest, including gland units, lumina, and stroma. Each block was processed by the cardinal multiridgelet transform. Features were extracted from each block for consideration in developing SVM classifiers for discriminating Gleason grade 3 versus grade 4. Three classifiers have been developed based on features selected and the adoption of training samples. 
Classifier A: Images of grade P3S3 and P4S4 are regarded as having homogeneous characteristics in various regions of an image, and texture feature extracted from each of the 9 patches ( 9 blocks of 256x256 subimages) may give a representative feature vector for the same Gleason grade of that image. The 16 sample images of P3S3 and 16 sample images of P4S4 may provide 144 (16x9) training patterns for each class. This will allow us to select sufficient number of features for training a RBF SVM classifier. The classifier will be validated with the 9-fold cross-validation and tested by the remaining 16 P4S4 images (with 144 testing patterns).

Classifier B: Each feature extracted from a patch is averaged over the 9 patches of an image to give a feature vector for that sample image. In this case, only 32 training patterns are available. Thus, the number of features used must be limited to not more than 3 or 4 . The trained classifier will be validated with the LOO cross-validation technique and tested by 16 patterns from the remaining 16 images.

Classifier C: Add 18 sample images of P3S4 into the pool of 16 sample images of P3S3 to form a set of 34 training samples of class P3 (primary Gleason grade 3), and combining 12 sample images of P4S3 with 22 sample images of P4S4 to form a set of 34 training samples of class P4 (primary Gleason grade 4). Features are averaged over 9 patches in an image, as done in Classifier B. There are 68 training samples available, thus, more features may be used in this case for discriminating class P3 and class P4. The classifier will be validated with the LOO cross-validation and tested by 10 remaining samples of P4S4. 


\subsection{EXPERIMENTAL RESULTS}

\subsubsection{Classifier A}

Six features were selected: four variance features from multiridgelet packets $0,1,2$, and 3 and two entropy features from packets 4 and 6. $\underline{x}=\left(v_{0}, v_{1}, v_{2}, v_{3}, s_{4}, s_{6}\right)^{T}$ Note that the packet index is frequency indexed. 144 patch-based training patterns for P3S3 and 144 training patterns for P4S4 were used to train the classifier of RBF SVM. The parameter of the Gaussian kernel was $\sigma=0.28$. The SVM has 115 support vectors. Cross-validation was performed with the 9-fold method where the 9 patterns associated with the same image were pulled out for each time. The testing set contains of 144 patterns from 10 P4S4 images.

The training and testing result is given in Table 5. The 9-fold cross-validation test was $75 \%$, and the classification test was $73.61 \%$. This result was based on the individual patch in an image. It is encouraging to note that the classification of an image should be made on the basis of the majority (6-to-3) decision of 9 patches in the image, the testing accuracy would be up to 90\%. For comparison, a similar RBF SVM classifier based on the curvelet method using 8 features gave $54.86 \%$ 9-fold cross-validation accuracy and $65.63 \%$ testing set accuracy. The support vectors, weights $\left(w_{i}=\alpha_{i} z_{i}\right)$, and threshold of the RBF SVM Classifier A are listed in Table 8 and Table 9 in Appendix A.

The area under ROC (AUC) curve and its 95\% confidence interval based on 9-fold cross-validation test were determined using DBM-MRMC software with PROPROC fit. The ROC fitting curves are shown in Figure 24. AUC of the CMRT-based classifier is significantly higher than that of the curvelet-based one well above $95 \%$ confidence interval. 
Table 5. Training and testing results of RBF SVM Classifier A for prostate cancer Gleason grade 3 versus grade 4, the result of the cardinal multiridgelet-based method listed in the first row and that of the curvelet-based method is in the second row.

\begin{tabular}{|c|c|c|c|c|c|c|}
\hline & SVM Sigma & \# SV & Training & 9-fold CV & AUC & Test \\
\hline CMRT & 0.28 & 115 & $100.00 \%$ & $75.00 \%$ & $\begin{array}{c}0.8857 \\
(0.8518,0.9197)\end{array}$ & $73.61 \%$ \\
\hline Curvelets & 0.25 & 121 & $100.00 \%$ & $54.86 \%$ & $\begin{array}{c}0.7011 \\
(0.6551,0.7471)\end{array}$ & $65.63 \%$ \\
\hline
\end{tabular}

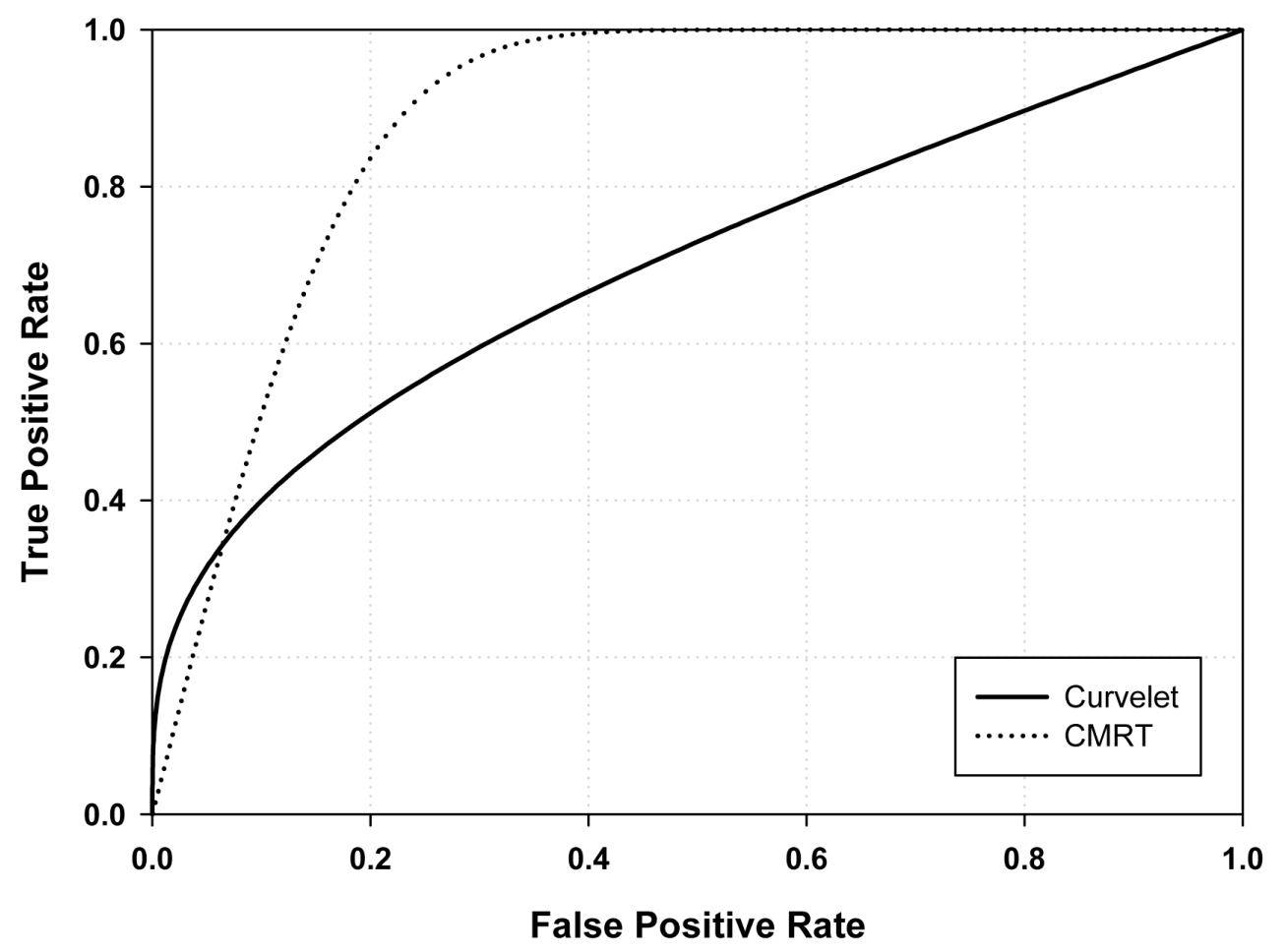

Figure 24 ROC curves of the validation test results of Classifier A for prostate cancer Gleason grade 3 versus grade 4 with PROPROC fitting method. 


\subsubsection{Classifier B}

In this case, features from 9 patches (blocks) were averaged for each image, only 32 testing samples (16 P3S3 and $16 \mathrm{P} 4 \mathrm{~S} 4)$ were available. Due to the small population of training samples, only three features were selected: variance features from multiridgelet packets 1 and 2 , and entropy features from packet $6, \underline{x}=\left(v_{1}, v_{2}, s_{6}\right)$. The RBF SVM Classifier B was trained. The Gaussian kernel parameter was chosen as $\sigma=0.15$. The SVM has 17 support vectors. A curvelet-based SVM Classifier B was also studied for comparison where two variance features from two lower frequency scales and two entropy features from two higher frequency scales were selected. For both classifiers, LOO cross-validation results and 10 P4S4 test results are given in Table 6; as shown, the performance of the CMRT-based Classifier B was remarkable with the test accuracy of $100 \%$. The AUC measure was 0.9651 with a $95 \%$ confidence interval of $(0.9090,1.0000)$ which is well above the AUC value 0.8316 of curvelet-based classifier. Support vectors, weights, and threshold of SVM for both CMRT-based and curvelet-based Classifier B are listed in Table 10 and Table 11 in the Appendix A. Superior performance of the CMRT-based Classifier B is also demonstrated by the area under ROC curves shown in Figure 25. 
Table 6. Training and testing results of RBF SVM Classifier B for prostate cancer Gleason grade 3 versus grade 4.

\begin{tabular}{|c|c|c|c|c|c|c|}
\hline & SVM Sigma & \# SV & Training & LOO CV & AUC & Test \\
\hline CMRT & 0.15 & 17 & $100.00 \%$ & $93.75 \%$ & $\begin{array}{c}0.9651 \\
(0.9090,1.0000)\end{array}$ & $100.00 \%$ \\
\hline Curvelets & 0.3 & 15 & $100.00 \%$ & $81.25 \%$ & $\begin{array}{c}0.8316 \\
(0.6671,0.9961)\end{array}$ & $70.00 \%$ \\
\hline
\end{tabular}

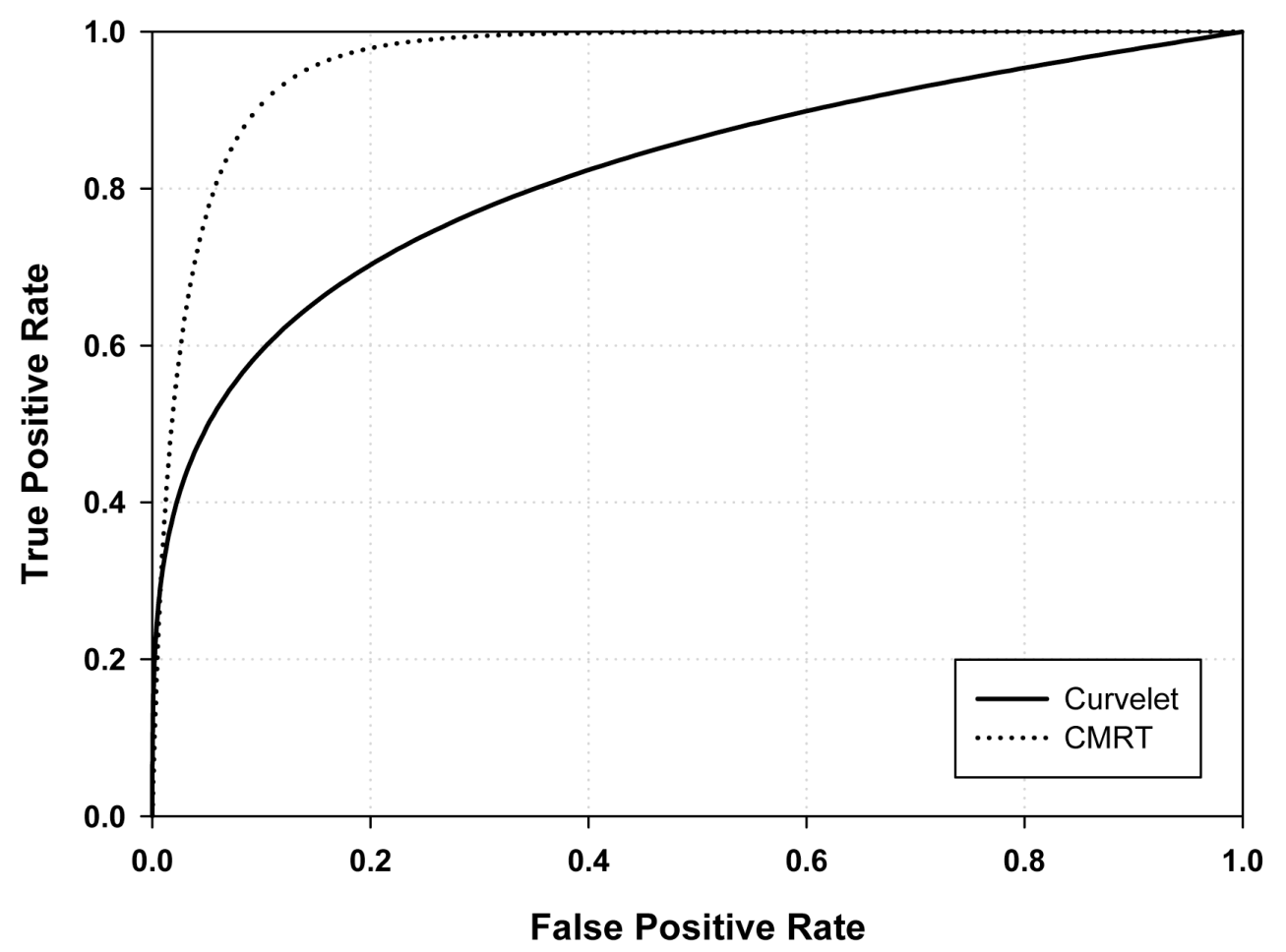

Figure 25 ROC curves of LOO validation test of Classifier B obtained by using PROPROC fitting (with 16 P3S3 and 16 P4S4) 


\subsubsection{Classifier $\mathrm{C}$}

In consideration of increasing the size of training samples from our available database, 18 images of P3S4 were added to the pool of 16 images of P3S3 to form a set P3 training samples of grade 3, $12 \mathrm{P} 4 \mathrm{~S} 3$ images were added to $22 \mathrm{P} 4 \mathrm{~S} 4$ images to form a set $\mathrm{P} 4$ of training samples of grader 4. 10 remaining P4S4 images were taken as testing samples. Because there involved a number of borderline samples, a stable classification boundary may be more difficult to find. Six selected features were from variance features from multiridgelet packets $0,1,2$, and 3 again, and two entropy features from packets 1 and 2, which were found more discriminative than those from packets 4 and $6, \underline{x}=\left(v_{0}, v_{1}, v_{2}, v_{3}, s_{1}, s_{2}\right)$. Gaussian kernel $\sigma$ was chosen as 0.24. The successful training gave 42 support vectors. The LOO cross-validation accuracy was $86.76 \%$ and the test using the 10 P4S4 samples was $90 \%$, which is 9 out of 10 correct classification of the samples. The ROC curves of the validation test was shown in Figure 26 and $\mathrm{AUC}=0.8802$. A curvelet-based 8-feature Classifier $\mathrm{C}$ was also trained and tested, the numbers are given in Table 7. Support vectors, weights, and threshold of SVM for both CMRT-based and curvelet-based Classifier C are listed in Table 12 and Table 13 in Appendix A. 
Table 7 Training and testing results of RBF SVM Classifier C for prostate cancer Gleason grade 3 versus grade 4.

\begin{tabular}{|c|c|c|c|c|c|c|}
\hline & SVM Sigma & \# SV & Training & LOO CV & AUC & Test \\
\hline CMRT & 0.24 & 42 & $100.00 \%$ & $86.76 \%$ & $\begin{array}{c}0.8802 \\
(0.7806,0.9799)\end{array}$ & $90.00 \%$ \\
\hline Curvelets & 0.3 & 45 & $100.00 \%$ & $72.06 \%$ & $\begin{array}{c}0.7451 \\
(0.6219,0.8683)\end{array}$ & $40.00 \%$ \\
\hline
\end{tabular}

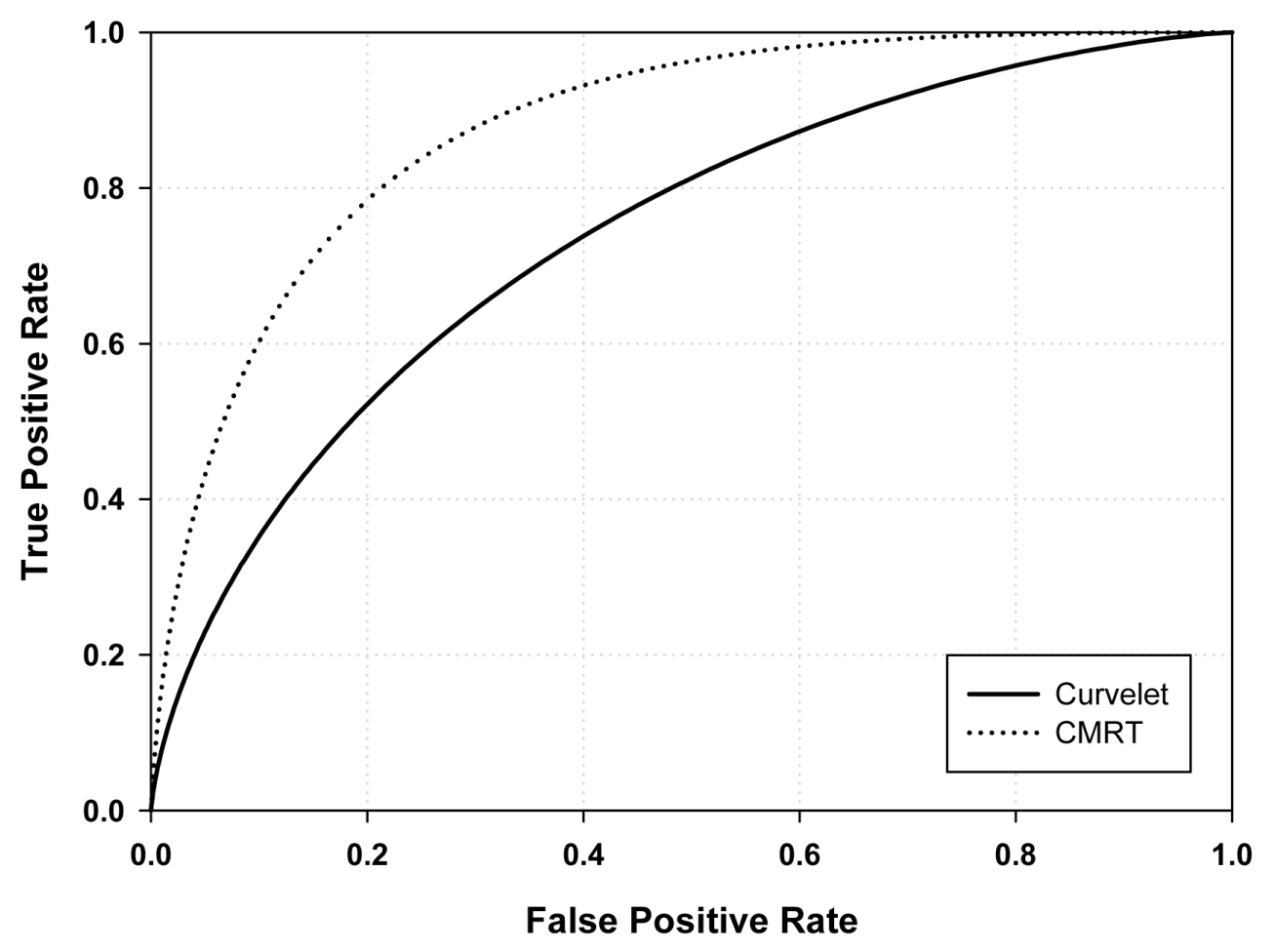

Figure 26 ROC curves of LOO validation test of Classifier C obtained by using PROPROC fitting (with 34 P3 and 34 P4 cases). 


\subsection{DISCUSSION}

The three classifiers presented together show the high potential of the CMRT-based texture classification of prostate cancer histological images for classifying Gleason grades. In reference to the results reported in the literature, it is worthy to note the recent contributions by Doyle et al. [57], by Huang et al. [61], by Khurd, et al. [63], and by Nguyen et al. [64]. For classification of Gleason grade 3 and grade 4, the work of Doyle et al. was based on the use of 11 training samples of grade 3 and 7 training samples of grade 4 , which is a smaller training set than what we have use. Huang considered a 4-class (grades 1-2, 3, 4 and 5) RBF SVM classifier using 5 fractal dimension based features, the machine was trained with 50 samples of grade 1-2, 72 samples of grade 3, 31 samples of grade 4, and 52 samples of grade 5, the overall 4-class test accuracy was $94.6 \%$ but specific test results for classification between grade 3 and grade 4 was not given. Khurd et al. used the random texton forest approach and gave a high testing accuracy of their RBF SVM classifier. It was trained with 15 samples of grade 3 and 15 samples of grade 4. The test accuracy was $94.0 \%$ with $\mathrm{AUC}=0.976$ for testing with 10 samples of grade 3 and 35 samples of grade 4. Nguyen et al. extracted 9 statistical features from segmented regions and trained a linear SVM classifier with 28 samples of grade 3 and 20 samples of grade 4, the two-

class classification accuracy was $87.3 \%$. Our CMRT-based classifier B performed remarkably well but for a limited test set of only 10 samples of P4S4. Nevertheless, it presents a great impetus to develop further with a larger set of training and testing samples. Gland units and the corresponding cells form a unique structure that determines the cancer grade of the prostate histological images. The approximate translation invariance and rotation invariance properties of the CMRT plus the finer frequency partition of the CMRT packet decomposition may give a 
more flexible feature extraction for achieving better classification accuracy than the curvelet transform based method. 


\subsection{CONCLUSIONS AND SUGGESTIONS}

One major contribution in this dissertation is the development of the cardinal multiridgelet transform (CMRT) for texture analysis where the cardinal multiwavelets utilized in the ridgelet transform are considered as nearly an approximate Hilbert transform pair. This is supported by the computational result that Fourier transforms of the two cardinal scaling filters have almost the same magnitude response in their pass-band and the difference between their group delays and between their phase delays in the pass-band are nearly one half sample, the similar result also holds for two cardinal wavelet filters. In two experiments, when the pair of decomposition coefficients from the CMRT at each scale level are combined in the sum of squares form, they were shown to have nearly the same energy regardless the shift of the input signal. This leads to our claim that the CMRT is nearly approximately shift invariant. This enables the CMRT to gain three advantages, which will be advantageous for texture analysis. First, a cardinal orthogonal multiscaling function assures to achieve minimal aliasing error during signal decomposition, which will result in a more faithful texture description in the transform domain. Secondly, because the pair of CMRT coefficients from two channels may be regarded as forming a complex coefficient, we can achieve approximate translation invariance of CMRT. Thirdly, a translation in image plane is reflected to a sinusoidal shift in variable $\rho$ in the Radon domain and a rotation of an off-center object also results in a sinusoidal shift in variable $\rho$ in addition to a shift in variable $\theta$; as the CMRT is almost translation invariant, thus it is also 
approximately rotation invariant. The CMRT also inherits some useful properties available in the single ridgelet transform and the Radon transform, such as highly anisotropic directional selectivity and robustness to noise. These are its merits in image texture analysis. Three studies on its applications have been conducted: texture database retrieval, LCD mura defect detection, and prostate cancer tissue image classification, all showed superior performances in comparison to those using curvelet transform, or ridgelet transform or Gabor wavelet transform.

The second major contribution of the thesis is the machine classification of prostate cancer histological images on discriminating Gleason grade 3 and grade 4 which is important in clinical decision affecting prognosis and treatment. The cardinal multiridgelet packet transform was applied in texture feature extraction from each patch of image data. Take nine patches to cover a major portion of each specimen image, the average of the nine sets of patch features were taken as the image features which were then selected, Sample images were obtained from the issue microarray TMA-471 under the courtesy of Johns Hopkins Medical School, 16 images of P3S3 ad 16 images of P4S4 were used as training samples. One support vector machine with Gaussian kernel was successfully trained and tested by the leave-one-out cross validation giving an accuracy of $93.75 \%$ which is very competitive among the currently reported results. 


\subsection{SUGGESTIONS FOR FURTHER RESEARCH}

The following two problems are suggested for further research:

1. More analysis and evaluation of the CMRT on texture analysis in comparison to dual-tree complex ridgelet transform. -- The use of oversampled CMRT and the trade-off between cardinality and symmetry of wavelets in texture feature extraction are worthy to be investigated.

2. Further development on the automatic classification of prostate cancer Gleason grading. -- When more sample images of Gleason grade 3 and grade 4, and sample images of other grades, are available in the database, more features can be used in the classifier, which will be retrained to have more accuracy and reliability. The CMRT-based texture segmentation of prostate histological images will be investigated to facilitate the recognition and classification of the transitional phase P3S4 and P4S3. Multi-class machine can be developed toward the goal of providing the machine-aided Gleason grading in pathology laboratories. 
APPENDIX A

RADIAL BASIS FUNCTION NON-LINEAR SUPPORT VECTOR MACHINE PARAMETERS FOR PROSTATE CANCER CLASSIFICATION STUDY 
Table 8 Parameters of the CMRT-based RBF SVM Classifier A for prostate cancer Gleason grade 3 versus grade 4

(16 P3S3 and 16 P4S4, 9 patches per each image).

(a) 6-feature support vectors

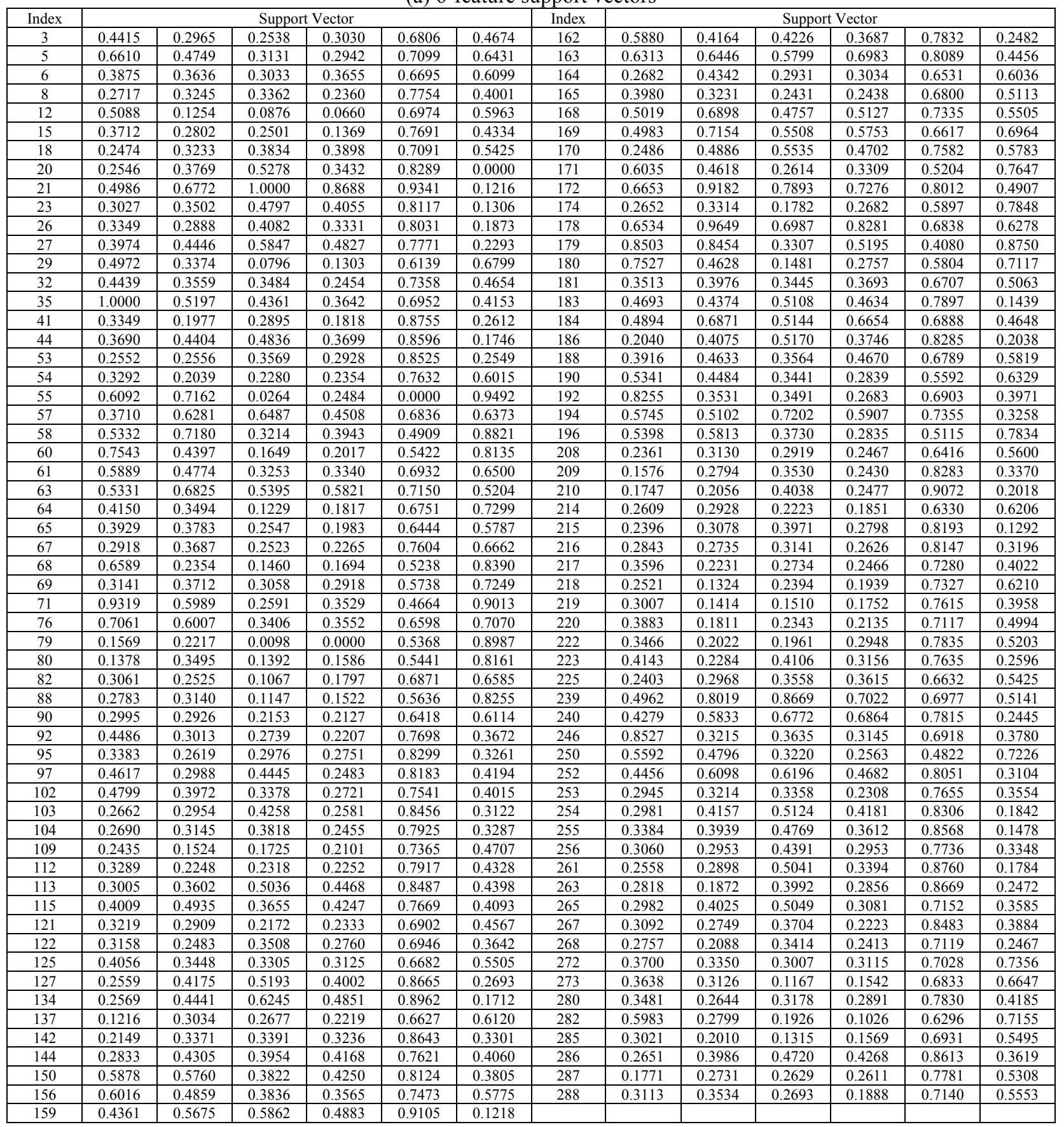

Pattern index 1 to 144 : Gleason grade 3

145 to 288 : Gleason grade 4 
(b) weights

(x e+03)

\begin{tabular}{|c|c|c|c|c|c|c|c|c|c|c|c|c|c|}
\hline Index & 3 & 5 & 6 & 8 & 12 & 15 & 18 & 20 & 21 & 23 & 26 & 27 & 29 \\
\hline Weight & -1.7045 & -0.0708 & -0.8672 & -5.5544 & -0.1718 & -0.9039 & -2.4687 & -0.2921 & -0.0071 & -0.4598 & -0.5264 & -0.3241 & -0.1292 \\
\hline Index & 32 & 35 & 41 & 44 & 53 & 54 & 55 & 57 & 58 & 60 & 61 & 63 & 64 \\
\hline Weight & -1.2085 & -0.0386 & -0.2508 & -1.5029 & -2.8714 & -1.3383 & -0.0024 & -0.1137 & -0.0392 & -0.051 & -0.5457 & -0.2484 & -0.0437 \\
\hline Weight & -2.4211 & -0.6366 & -0.0505 & -0.622 & -0.006 & -0.0265 & -0.0405 & -0.0085 & -0.6944 & -0.0152 & -3.8746 & -0.0702 & -2.2009 \\
\hline Index & 97 & 102 & 103 & 104 & 109 & 112 & 113 & 115 & 121 & 122 & 125 & 127 & 134 \\
\hline Weight & -0.1593 & -0.0792 & -1.6184 & -4.9936 & -0.9071 & -3.7085 & -0.5391 & -0.1436 & -3.7563 & -2.5568 & -0.4161 & -1.4447 & -0.212 \\
\hline Weight & -0.8359 & -0.3919 & -0.65 & 0.0133 & 0.6157 & 0.1124 & 0.0568 & 0.0025 & 0.188 & 6.6755 & 0.0098 & 0.0917 & 0.2138 \\
\hline Index & 171 & 172 & 174 & 178 & 179 & 180 & 181 & 183 & 184 & 186 & 188 & 190 & 192 \\
\hline Weight & 0.0615 & 0.0023 & 0.5022 & 0.0047 & 0.0049 & 0.0523 & 0.9992 & 0.0988 & 0.0738 & 0.7105 & 0.2042 & 0.2021 & 0.0196 \\
\hline Index & 194 & 196 & 208 & 209 & 210 & 214 & 215 & 216 & 217 & 218 & 219 & 220 & 222 \\
\hline Weight & 0.0308 & 0.0523 & 0.9587 & 0.8071 & 0.1914 & 2.2615 & 0.1524 & 7.6189 & 2.5994 & 0.1649 & 0.642 & 0.0562 & 1.6538 \\
\hline Index & 223 & 225 & 239 & 240 & 246 & 250 & 252 & 253 & 254 & 255 & 256 & 261 & 263 \\
\hline Weight & 0.1362 & 2.4217 & 0.0072 & 0.0189 & 0.0169 & 0.1187 & 0.1527 & 5.8916 & 0.4578 & 2.0907 & 2.0973 & 0.5183 & 0.064 \\
\hline
\end{tabular}

(c) threshold

threshold

$-0.1986$ 
Table 9 Parameters of the curvelet-based RBF SVM Classifier A for prostate cancer Gleason grade 3 versus grade 4

(a) 8-feature support vectors

\begin{tabular}{|c|c|c|c|c|c|c|c|c|c|c|c|c|c|c|c|c|c|}
\hline Index & \multicolumn{8}{|c|}{ Support Vector } & Index & \multicolumn{8}{|c|}{ Support Vector } \\
\hline 4 & 0.6209 & 0.0409 & 02473 & 0.6400 & 0.4702 & 0.8099 & 05855 & 0.9703 & 125 & 0.5627 & 02044 & 02164 & & 0.4212 & 0.7284 & 03524 & 0.6918 \\
\hline 6 & 02292 & 0.0205 & 02848 & 0.6642 & 0.4763 & 0.705 & 02807 & 0.7173 & 126 & 0.441 & 04371 & 03105 & 0.6992 & 05088 & 0.7593 & 03449 & 0.6420 \\
\hline 7 & 05856 & 0.1533 & 03285 & 0.7050 & 05156 & 0.8284 & 05700 & 0.9213 & 129 & 02311 & 03486 & 0.1704 & 0.6436 & 03638 & 0.7612 & 0.1756 & 0.6070 \\
\hline 8 & 0.1636 & 0.0614 & 02399 & 0.6639 & 03544 & 0.7590 & 02845 & 0.7537 & 130 & 02280 & 02436 & 02362 & 0.717 & 0.4205 & 0.8154 & 03384 & 0.8050 \\
\hline 10 & 03030 & 0.0102 & 03281 & 0.726 & 0.637 & 09387 & 0.4795 & 0.9524 & 131 & 02742 & 05578 & 0.1911 & 0.6431 & 03479 & 0.7319 & 02740 & 0.6871 \\
\hline 13 & 02908 & 0.0000 & 03665 & 0.6396 & 0.6031 & 0.8170 & 05076 & 0.8899 & 134 & 0.4017 & 0.6790 & 03839 & 0.8252 & 05153 & 0.8813 & 0.4276 & 0.8041 \\
\hline 16 & 03242 & 0.0409 & 0.4611 & 0.8000 & 05656 & 0.8852 & 05338 & 0.9030 & 143 & 02678 & 02521 & 02794 & 0.727 & 0307 & 0.7245 & 02135 & 0.6177 \\
\hline 17 & 0.0116 & 0.0000 & 02971 & 05952 & 0.4611 & 0.7323 & 05000 & 0.8304 & 145 & 02863 & 0.0409 & 0.4215 & 0.7867 & 0.6637 & 09306 & 0.6033 & 09946 \\
\hline 18 & 0.024 & 0.0614 & 0.4789 & 0.7381 & 0.4816 & 0.7451 & 02997 & 0.7109 & 146 & 03426 & 02198 & 03615 & 0.7391 & 05748 & 0.8762 & 0.6283 & 09393 \\
\hline 20 & 02459 & 0.1840 & 02845 & 0.712 & 05584 & 0.8953 & 0.4554 & 0.8616 & 147 & 03460 & 0.1533 & 02206 & 0.6452 & 05507 & 0.8282 & 05058 & 0.8578 \\
\hline 21 & 02435 & 0.0102 & 0.5451 & 0.8208 & 09239 & 1.0000 & 0.6705 & 1.0000 & 150 & 0.4385 & 02828 & 02209 & 0.6644 & 0.4236 & 0.7933 & 03412 & 0.7955 \\
\hline 23 & 02345 & 0.0716 & 0.1894 & 0.6652 & 0.4906 & 0.8741 & 03671 & 0.8205 & 153 & 0.4381 & 02130 & 02253 & 0.6376 & 0.4803 & 0.8198 & 0.4285 & 0.8614 \\
\hline 29 & 0.0693 & 0.0102 & 0.0891 & 0.4770 & 02150 & 0.6287 & 0.1710 & 0.6342 & 156 & 0.1237 & 0.0920 & 02114 & 05790 & 03573 & 0.7083 & 02750 & 0.7231 \\
\hline 34 & 03279 & 0.0716 & 02194 & 0.6729 & 0.4568 & 0.8302 & 0.4778 & 0.9040 & 157 & 0.1916 & 0.1108 & 03095 & 0.7278 & 0.4935 & 0.8646 & 04329 & 0.8962 \\
\hline 43 & 0317 & 0.0409 & 0.1764 & 0.5854 & 0.4832 & 0.7994 & 0.4303 & 0.8499 & 159 & 0.4049 & 02794 & 02728 & 0.744 & 05084 & 0.8750 & 04269 & 0.8739 \\
\hline 45 & 0.4226 & 03486 & 02235 & 0.6894 & 0.4680 & 0.8526 & 0.4533 & 0.8758 & 160 & 0.4368 & 0.1125 & 03013 & 0.6533 & 0.4718 & 0.8032 & 05147 & 0.9032 \\
\hline 48 & 03302 & 0.1586 & 02694 & 0.6873 & 04880 & 0.7987 & 05049 & 0.8282 & 165 & 02007 & 0.0614 & 0287 & 0.6944 & 03208 & 0.729 & 0.1937 & 0.6505 \\
\hline 50 & 0.4995 & 03690 & 0.4281 & 0.8507 & 0.4686 & 0.8701 & 03994 & 0.8448 & 168 & 03986 & 0.0102 & 0.4079 & 0.7373 & 05266 & 0.8168 & 03828 & 0.7798 \\
\hline 51 & 02845 & 02334 & 02147 & 0.6107 & 04450 & 0.7629 & 05558 & 0.8347 & 169 & 0.6111 & 0.1636 & 0.4511 & 0.6215 & 0.6162 & 0.7128 & 0.6817 & 0.8096 \\
\hline 53 & 03648 & 03045 & 02178 & 0.6926 & 03791 & 0.8079 & 03410 & 0.8098 & 170 & 05891 & 05305 & 0.4800 & 0.7770 & 05786 & 0.7867 & 05627 & 0.7826 \\
\hline 54 & 0.1306 & 0.1279 & 02032 & 05530 & 03660 & 0.7086 & 0.4516 & 0.7775 & 171 & 0.9888 & 0.4852 & 02930 & 05959 & 03747 & 0.6822 & 03263 & 0.6232 \\
\hline 55 & 05510 & 0.0205 & 0.0000 & 0.0000 & 0.0000 & 0.0000 & 0.0000 & 0.0000 & 174 & 0.1418 & 0.0409 & 02140 & 05656 & 02546 & 0.5932 & 0.1920 & 05631 \\
\hline 56 & 05535 & 0.1902 & 0.1279 & 0.4522 & 0.0695 & 02848 & 0.0350 & 0.1788 & 176 & 0.4743 & 0.1415 & 03502 & 0.7213 & 05104 & 0.797 & 03690 & 0.7088 \\
\hline 57 & 03509 & 0.0818 & 03684 & 0.6666 & 05802 & 0.7588 & 0.4280 & 0.7095 & 179 & 0.6752 & 0.0614 & 03773 & 05505 & 0.4501 & 0.5843 & 02860 & 04501 \\
\hline 60 & 05743 & 0.1022 & 0.1660 & 05438 & 03781 & 0.6585 & 02915 & 0.6019 & 180 & 0.6130 & 0.1006 & 02021 & 05349 & 03995 & 0.6536 & 0.4086 & 0.6819 \\
\hline 61 & 0.6783 & 02726 & 03509 & 0.6950 & 05236 & 0.7267 & 03354 & 0.6823 & 181 & 03094 & 02248 & 02053 & 0.6654 & 03696 & 0.7425 & 02185 & 0.6045 \\
\hline 62 & 03723 & 0.0307 & 0.1741 & 05564 & 02550 & 05521 & 0.1790 & 0.4430 & 182 & 0.7848 & 02215 & 02884 & 0.7168 & 0.6156 & 0.8443 & 03543 & 0.7140 \\
\hline 63 & 0.4780 & 0.0716 & 0.4141 & 0.7179 & 0.6861 & 0.8331 & 0.4971 & 0.7946 & 183 & 0.6642 & 0.1636 & 02638 & 0.6808 & 05971 & 0.8773 & 0.4708 & 0.8387 \\
\hline 64 & 0.0878 & 0.0511 & 0.1533 & 0.5224 & 0.1973 & 05919 & 0.1697 & 0.6248 & 188 & 0.6107 & 0.4591 & 0.4462 & 0.7202 & 03921 & 0.7428 & 02233 & 0.6468 \\
\hline 65 & 0.1411 & 0.0511 & 02347 & 0.6171 & 02691 & 0.6628 & 0.1793 & 0.6111 & 189 & 0.6719 & 0.4870 & 02601 & 0.6734 & 0.4838 & 0.8197 & 0.4767 & 0.8357 \\
\hline 67 & 02005 & 0.1721 & 0.1452 & 05546 & 02991 & 0.6690 & 03852 & 0.7602 & 193 & 0.6420 & 03492 & 03215 & 0.6888 & 05372 & 0.7499 & 0327 & 05616 \\
\hline 68 & 0.8090 & 03101 & 0.1779 & 05394 & $02 \pi 7$ & 0.6136 & 02731 & 0.6353 & 196 & 0.7088 & 02027 & 0.1666 & 05562 & 03565 & 0.6660 & 02811 & 05492 \\
\hline 70 & 0.6701 & 0.1312 & 02533 & 0.5398 & 03569 & 0.6480 & 0.3500 & 0.7196 & 197 & 0.5442 & 02930 & 0.1687 & 0.6536 & 05445 & 0.9039 & 05348 & 0.8585 \\
\hline 71 & 09075 & 02044 & 02830 & 0.4411 & 0324 & 0.4967 & 03078 & 0.4701 & 198 & 0.8875 & 02930 & 03156 & 0.7041 & 0.6253 & 0.8614 & 0.6037 & 0.8813 \\
\hline 73 & 09309 & 1.0000 & 03145 & 0.6678 & 04168 & 0.6967 & 0.4383 & 0.7073 & 211 & 02058 & 03424 & 0.1980 & 0.6189 & 02758 & 0.6888 & 0.1196 & 05543 \\
\hline 74 & 0.7284 & 0.4104 & 02219 & 05662 & 03120 & 0.6355 & 03145 & 0.6198 & 214 & 03810 & 04529 & 02036 & 0.6055 & 03198 & 0.7189 & 0.1318 & 05715 \\
\hline 76 & 0.8472 & 03963 & 03999 & 0.6320 & 0.4840 & 0.6864 & 0.4061 & 0.7018 & 215 & 02056 & 03611 & 03286 & 0.7312 & 05211 & 0.8497 & 03612 & 0.7826 \\
\hline 80 & 03380 & 03900 & 02062 & 05595 & 02131 & 05664 & 0.1120 & 0.4772 & 21 & 0.0508 & 0.0511 & 0.1409 & 0.5458 & 0.4766 & 0.7858 & 03972 & 0.8083 \\
\hline 82 & 05413 & 0.4976 & 0.1712 & 0.6181 & 02457 & 0.6570 & 02683 & 0.6487 & 222 & 0.0280 & 0.0000 & 0.1434 & 0.5229 & 03798 & 0.7280 & 0.4363 & 0.7852 \\
\hline 90 & 0.7322 & 0.6092 & 02062 & 0.6725 & 03612 & 0.7594 & 02632 & 0.6616 & 225 & 0.0987 & 0.0511 & 02623 & 0.584 & 0.4953 & 0.7796 & 0.4835 & 0.8064 \\
\hline 92 & 03584 & 0.1619 & 02109 & 0.6277 & 04109 & 0.7812 & 03697 & 0.7982 & 234 & 0.0501 & 0.0102 & 0.1028 & 05772 & 03637 & 0.7801 & 02897 & 0.7875 \\
\hline 94 & 03801 & 0.1636 & 02162 & 05997 & 0.6360 & 0.8357 & 0.6196 & 0.8755 & 243 & 02321 & 0.0307 & 0.8935 & 0.9120 & 0.9194 & 0.8986 & 03871 & 0.6749 \\
\hline 95 & 03590 & 0.1619 & 0.1542 & 05895 & 03972 & 0.8067 & 0.4030 & 0.8487 & 254 & 0.1866 & 0.1415 & 02425 & 0.6916 & 0.4638 & 0.8805 & 03606 & 0.8439 \\
\hline 98 & 05749 & 03628 & 03289 & 0.7497 & 05084 & 0.8682 & 0.4565 & 0.8679 & 255 & 0.1806 & 0.0716 & 02610 & 0.6945 & 05733 & 0.8784 & 0.4835 & 0.8815 \\
\hline 101 & 03416 & 0.2709 & 0.1484 & 0.6588 & 03638 & 0.8242 & 03893 & 0.8400 & 257 & 02582 & 0.0920 & 0.1168 & 0.5886 & 0.4384 & 0.8476 & 03455 & 0.8369 \\
\hline 103 & 03125 & 02317 & 0.1926 & 0.6456 & 04560 & 0.8316 & 0.4373 & 0.8228 & 258 & 0.1659 & 0.1431 & 02375 & 0.6876 & 0.4779 & 0.8597 & 0384 & 0.8495 \\
\hline 105 & 0.6868 & 02726 & 0.1882 & 0.6218 & 03536 & 0.7421 & 03210 & 0.7446 & 265 & 02062 & 0.0716 & 02143 & 0.6477 & 0.4997 & 0.8502 & 03080 & 0.7604 \\
\hline 106 & 05262 & 02436 & 0.1292 & 0.6104 & 03805 & 0.7982 & 03947 & 0.8145 & 268 & 03856 & 0.0920 & 0.0749 & 05739 & 0.4497 & 0.8611 & 03827 & 0.8257 \\
\hline 107 & 05149 & 03424 & 02096 & 0.6178 & 03650 & 0.712 & 03669 & 0.8043 & 269 & 03049 & 0.0511 & 0.0770 & 0577 & 0.4534 & 0.8645 & 03765 & 0.8530 \\
\hline 109 & 0.0936 & 0.0409 & 0.1085 & 05618 & 03642 & 0.7575 & 03087 & 0.7634 & 272 & 0.0626 & 0.0000 & 03142 & 05450 & 0.4608 & 0.6301 & 0.4278 & 0.7166 \\
\hline 110 & 0.0338 & 0.0102 & 0.1498 & 05811 & 0,4208 & 0.7612 & 0.4044 & 0.8064 & 273 & 0.1540 & 0.0102 & 0.1239 & 05690 & 02035 & 0.6256 & 02105 & 0.6487 \\
\hline 111 & 0.4235 & 0.0307 & 02134 & 0.6179 & 0.4926 & 0.7990 & 05073 & 0.8313 & 275 & 0.1700 & 0.1415 & 0.0767 & 05065 & 02832 & 0.6731 & 03224 & 0.7473 \\
\hline 113 & 0.1218 & 0.0920 & 02842 & 0.6579 & 0.6230 & 0.8334 & 0.7050 & 0.9170 & 27 & 0.1032 & 0.1533 & 02477 & 0.6293 & 05023 & 0.7603 & 05249 & 0.8331 \\
\hline 114 & 0.1496 & 0.0614 & 0.0960 & 05559 & 02497 & 0.6940 & 02812 & 0.7432 & 279 & 03758 & 03355 & 0.1243 & 0.4710 & 02517 & 0.6797 & 0.4279 & 0.8574 \\
\hline 117 & 0.4299 & 0.1022 & 03301 & 0.7273 & 04123 & 0.7930 & 03392 & 0.7988 & 280 & 02648 & 0.1125 & 0.1936 & 0.6267 & 0.4302 & 0.7550 & 0.4396 & 0.7846 \\
\hline 119 & 03967 & 0.0511 & 0.0416 & 0.4556 & 02131 & 0.6232 & 0.1733 & 0.6069 & 282 & 0.6525 & 02607 & 0.1693 & 05691 & 03039 & 0.6789 & 03131 & 0.6781 \\
\hline 120 & 02481 & 0.0409 & 02510 & 0.6183 & 0.4304 & 0.7506 & 02519 & 0.6475 & 283 & 02179 & 02317 & 0.1873 & 0.6393 & 03511 & 0.7550 & 03645 & 0.7802 \\
\hline 121 & 0.1557 & 0.1125 & 0.1834 & 05930 & 04451 & 0.724 & 03484 & 0.7581 & 286 & 02927 & 02027 & 0.4450 & 0.7550 & 05578 & 0.8204 & 0.6610 & 0.8981 \\
\hline 122 & 0.1534 & 0.1227 & 0.1743 & 0.6068 & 03896 & 0.7679 & 03148 & 0.7427 & 287 & 03218 & 03265 & 02480 & 0.6541 & 03724 & 0.7168 & 03887 & 0.7705 \\
\hline 123 & 02117 & 0.1533 & 03091 & 0.6623 & 0.6892 & 0.8160 & 03198 & 0.6796 & 288 & 0.6993 & 05430 & 0.1862 & 0.6637 & 02996 & 0.7262 & 02264 & 0.7085 \\
\hline 124 & 0.6518 & 03509 & 02793 & 0.6804 & 05636 & 0.8034 & 0.4384 & 0.7448 & & & & & & & & & \\
\hline
\end{tabular}

Pattern index 1 to 144 : Gleason grade 3

145 to 288 : Gleason grade 4 
(b) weights

\begin{tabular}{|c|c|c|c|c|c|c|c|c|c|c|c|c|c|}
\hline Index & 4 & 6 & 7 & 8 & 10 & 13 & 16 & 17 & 18 & 20 & 21 & 23 & 29 \\
\hline Weight & -0.722 & -111.208 & -47.435 & -127.746 & -7.775 & -25.658 & -23.103 & -48.862 & -10.333 & -187.116 & -2.382 & -428.745 & -42.266 \\
\hline Index & 34 & 43 & 45 & 48 & 50 & 51 & 53 & 54 & 55 & 56 & 57 & 60 & 61 \\
\hline Weight & -17.430 & -105.708 & -59.797 & -288.228 & -3.774 & -72.165 & -58.472 & -30.791 & -2.467 & -0.022 & -8.926 & -20.983 & -10.973 \\
\hline Index & 62 & 63 & 64 & 65 & 67 & 68 & 70 & 71 & 73 & 74 & 76 & 80 & 82 \\
\hline Weight & -12.644 & -6.798 & -72.999 & -264.088 & $\begin{array}{l}-397.578 \\
\end{array}$ & -29.935 & -8.778 & -0.579 & -2.377 & -2.197 & -2.294 & -16.698 & -18.869 \\
\hline Index & 90 & 92 & 94 & 95 & 98 & 101 & 103 & 105 & 106 & 107 & 109 & 110 & 111 \\
\hline Weight & -24.085 & -245.605 & -36.764 & -77.620 & -64.426 & -7.658 & -57.401 & -13.852 & -10.056 & -40.148 & -249.866 & -155.074 & -64.726 \\
\hline Index & 113 & 114 & 117 & 119 & 120 & 121 & 122 & 123 & 124 & 125 & 126 & 129 & 130 \\
\hline Weight & -22.809 & -137.659 & -0.856 & -16.950 & -53.122 & $\begin{array}{l}-556.694 \\
\end{array}$ & -72.658 & -16.313 & -24.707 & -73.060 & -12.467 & -86.326 & -262.871 \\
\hline Index & 131 & 134 & 143 & 145 & 146 & 147 & 150 & 153 & 156 & 157 & 159 & 160 & 165 \\
\hline Weight & -1.816 & -6.937 & -68.737 & 10.673 & 22.117 & 206.324 & 190.933 & 11.595 & 426.853 & 16.145 & 172.269 & 147.411 & 155.793 \\
\hline Index & 168 & 169 & 170 & 171 & 174 & 176 & 179 & 180 & 181 & 182 & 183 & 188 & 189 \\
\hline Weight & 46.974 & 8.829 & 9.168 & 7.565 & 112.212 & 19.793 & 1.043 & 45.289 & 67.988 & 4.135 & 11.436 & 4.645 & 40.158 \\
\hline Index & 193 & 196 & 197 & 198 & 211 & 214 & 215 & 221 & 222 & 225 & 234 & 243 & 254 \\
\hline Weight & 18.638 & 8.713 & 16.432 & 4.310 & 58.579 & 16.999 & 64.035 & 63.754 & 126.194 & 41.540 & 193.677 & 0.113 & 161.420 \\
\hline Index & 255 & 257 & 258 & 265 & 268 & 269 & 272 & 273 & 275 & 277 & 279 & 280 & 282 \\
\hline Weight & 120.425 & 141.448 & 46.807 & 456.846 & 18.932 & 26.388 & 7.186 & 166.322 & 233.725 & 26.564 & 24.009 & 689.112 & 69.151 \\
\hline Index & 283 & 286 & 287 & 288 & & & & & & & & & \\
\hline Weight & 436.082 & 23.205 & 13.406 & 26.825 & & & & & & & & & \\
\hline
\end{tabular}

(c) threshold

threshold

1.4772 
Table 10 Parameters of the CMRT-based RBF SVM Classifier B for prostate cancer Gleason grade 3 versus grade 4 (16 P3S3 and 16 P4S4, features from the average of 9 patches of an image).

\begin{tabular}{|c|c|c|c|c|c|}
\hline Index & \multicolumn{3}{|c|}{ 3-feature Support Vectors } & Weights & Threshold \\
\hline 3 & 0.4857 & 0.4424 & 0.1402 & -2.6943 & 0.3912 \\
\hline 4 & 0.5206 & 0.3522 & 0.4541 & -1.0269 & \\
\hline 7 & 0.8799 & 0.6224 & 1.0000 & -1.4699 & \\
\hline 9 & 0.5055 & 0.1740 & 0.9452 & -1.0330 \\
\hline 10 & 0.3556 & 0.2004 & 0.7533 & -1.3915 \\
\hline 12 & 0.3861 & 0.2948 & 0.2394 & -2.3887 \\
\hline 14 & 0.4191 & 0.3976 & 0.4570 & -0.6575 \\
\hline 16 & 0.3930 & 0.2268 & 0.3781 & -4.7864 \\
\hline 17 & 0.8000 & 0.7476 & 0.0000 & 0.4201 \\
\hline 18 & 0.7902 & 0.8072 & 0.1392 & 0.3346 \\
\hline 20 & 0.9060 & 0.7966 & 0.7358 & 0.7439 & \\
\hline 22 & 0.5483 & 0.4642 & 0.3527 & 3.2445 & \\
\hline 23 & 0.0000 & 0.0000 & 0.1617 & 0.4934 & \\
\hline 24 & 0.3172 & 0.2058 & 0.3232 & 5.8969 & \\
\hline 27 & 1.0000 & 1.0000 & 0.4213 & 0.5715 \\
\hline 29 & 0.4292 & 0.3468 & 0.0124 & 2.4418 \\
\hline 31 & 0.2733 & 0.1460 & 0.5603 & 1.3014 & \\
\hline
\end{tabular}

Pattern index 1 to 16 : Gleason grade 3

17 to 32 : Gleason grade 4

Table 11 Parameters of the curvelet-based RBF SVM Classifier B for prostate cancer Gleason grade 3 versus grade 4 (16 P3S3 and 16 P4S4, features from the average of 9 patches of an image).

\begin{tabular}{|c|c|c|c|c|c|c|}
\hline Index & \multicolumn{4}{|c|}{ 4-feature Support Vectors } & Weights & Threshold \\
\hline 1 & 0.6978 & 0.2715 & 0.7129 & 0.8080 & -11.5511 & 1.3364 \\
\hline 3 & 0.2688 & 0.2779 & 1.0000 & 0.7744 & -8.4969 & \\
\hline 4 & 0.4596 & 0.1228 & 0.5536 & 0.5854 & -56.0298 & \\
\hline 7 & 0.6571 & 0.2341 & 0.0000 & 0.0000 & -1.5807 & \\
\hline 8 & 0.7048 & 0.2024 & 0.2318 & 0.2743 & -0.8730 & \\
\hline 9 & 0.8080 & 0.1789 & 0.0911 & 0.1066 & -0.1793 & \\
\hline 13 & 0.3131 & 0.1996 & 0.7090 & 0.7727 & -33.4909 & \\
\hline 14 & 0.4423 & 0.2066 & 0.6419 & 0.5283 & -20.9858 & \\
\hline 15 & 0.3962 & 0.2957 & 0.7813 & 0.5679 & -0.8780 & \\
\hline 18 & 0.4265 & 0.3427 & 0.9364 & 0.9878 & 9.9646 & \\
\hline 21 & 0.8438 & 0.4313 & 0.8389 & 0.5495 & 1.9276 & \\
\hline 22 & 0.9889 & 0.2646 & 0.8558 & 0.6453 & 2.2524 & \\
\hline 24 & 0.2844 & 0.1985 & 0.6752 & 0.5721 & 34.2223 & \\
\hline 30 & 0.2504 & 0.0573 & 0.8965 & 0.8369 & 13.2421 & \\
\hline 32 & 0.4883 & 0.1838 & 0.5492 & 0.6437 & 72.4565 & \\
\hline
\end{tabular}

Pattern index 1 to 16 : Gleason grade 3

17 to 32 : Gleason grade 4 
Table 12 Parameters of CMRT-based RBF SVM Classifier C for prostate cancer Gleason grade 3 versus grade 4

(34 P3 and $34 \mathrm{P} 4$, average features from 9 patches of an image).

\begin{tabular}{|c|c|c|c|c|c|c|c|}
\hline Index & \multicolumn{6}{|c|}{ 6-feature Support Vectors } & Weights \\
\hline 2 & 0.5207 & 0.3904 & 0.5059 & 0.5056 & 0.3582 & 0.3111 & -2.6145 \\
\hline 3 & 0.4290 & 0.4857 & 0.3346 & 0.5409 & 0.4424 & 0.2086 & -14.2418 \\
\hline 6 & 0.4367 & 0.4465 & 0.2018 & 0.5179 & 0.3363 & 0.1608 & -22.8745 \\
\hline 7 & 0.8287 & 0.8799 & 0.6489 & 0.2820 & 0.6224 & 0.8737 & -1.2331 \\
\hline 9 & 0.5365 & 0.5055 & 0.5536 & 0.0866 & 0.1740 & 0.6778 & -0.3937 \\
\hline 10 & 0.4167 & 0.3556 & 0.4599 & 0.1019 & 0.2004 & 0.4167 & -3.5994 \\
\hline 11 & 0.7782 & 0.6695 & 0.0102 & 0.7564 & 0.6085 & 0.0402 & -5.9980 \\
\hline 12 & 0.6065 & 0.4347 & 0.8457 & 0.2105 & 0.2906 & 0.7283 & -1.7755 \\
\hline 13 & 0.8104 & 0.5659 & 0.5437 & 0.2660 & 0.3917 & 0.7174 & -0.3486 \\
\hline 14 & 0.9412 & 0.5871 & 1.0000 & 0.0000 & 0.2638 & 1.0000 & -0.8268 \\
\hline 15 & 0.4388 & 0.2666 & 0.4869 & 0.3885 & 0.2429 & 0.3152 & -48.0859 \\
\hline 17 & 0.6260 & 0.4615 & 0.2011 & 0.3733 & 0.4314 & 0.2322 & -13.5838 \\
\hline 20 & 0.8354 & 0.3758 & 0.6677 & 0.0185 & 0.0611 & 0.7126 & -0.6008 \\
\hline 21 & 0.5855 & 0.4757 & 0.2631 & 0.5213 & 0.3895 & 0.2398 & -14.7456 \\
\hline 24 & 0.5097 & 0.4191 & 0.4385 & 0.3600 & 0.3976 & 0.3629 & -6.2532 \\
\hline 25 & 0.3167 & 0.4630 & 0.1586 & 0.4858 & 0.3338 & 0.1593 & -1.0974 \\
\hline 26 & 0.2153 & 0.3930 & 0.1908 & 0.4033 & 0.2268 & 0.1582 & -6.6003 \\
\hline 27 & 0.6003 & 0.3739 & 0.1630 & 0.3683 & 0.3146 & 0.1832 & -4.5787 \\
\hline 31 & 0.7158 & 0.6815 & 0.6179 & 0.2579 & 0.4777 & 0.7452 & -0.1930 \\
\hline 33 & 0.4322 & 0.3086 & 0.3274 & 0.2190 & 0.2134 & 0.3527 & -7.7438 \\
\hline 34 & 0.3483 & 0.3707 & 0.3641 & 0.1253 & 0.1937 & 0.3723 & -3.3986 \\
\hline 35 & 0.4996 & 0.4665 & 0.0494 & 0.8210 & 0.6260 & 0.0000 & 2.4840 \\
\hline 36 & 0.2888 & 0.3318 & 0.1818 & 0.7163 & 0.4084 & 0.1684 & 0.4341 \\
\hline 37 & 0.5303 & 0.4409 & 0.1212 & 0.4001 & 0.3594 & 0.2042 & 21.9117 \\
\hline 38 & 0.3820 & 0.3555 & 0.3191 & 0.4711 & 0.2604 & 0.1942 & 10.6966 \\
\hline 39 & 0.9332 & 0.7580 & 0.0000 & 0.7802 & 0.7724 & 0.1215 & 3.2053 \\
\hline 42 & 0.4690 & 0.4045 & 0.4381 & 0.3658 & 0.2869 & 0.3296 & 16.9594 \\
\hline 43 & 0.4933 & 0.5146 & 0.0379 & 0.4826 & 0.4206 & 0.1255 & 0.2673 \\
\hline 47 & 0.6196 & 0.6428 & 0.6564 & 0.4667 & 0.5259 & 0.4554 & 1.2258 \\
\hline 48 & 0.8957 & 0.9060 & 0.8879 & 0.4883 & 0.7966 & 0.7161 & 1.1913 \\
\hline 50 & 0.7724 & 0.5483 & 0.3309 & 0.5171 & 0.4642 & 0.3752 & 1.4684 \\
\hline 53 & 0.4513 & 0.2566 & 0.6449 & 0.2883 & 0.2550 & 0.3811 & 11.7989 \\
\hline 56 & 0.3177 & 0.1495 & 0.4737 & 0.2949 & 0.1224 & 0.2923 & 5.1884 \\
\hline 57 & 0.7339 & 1.0000 & 0.2127 & 1.0000 & 1.0000 & 0.1710 & 0.7049 \\
\hline 59 & 0.4162 & 0.4292 & 0.2318 & 0.5575 & 0.3468 & 0.2313 & 12.5034 \\
\hline 60 & 0.4312 & 0.3155 & 0.4700 & 0.4276 & 0.2452 & 0.3435 & 19.7921 \\
\hline 61 & 0.4138 & 0.2733 & 0.7103 & 0.1604 & 0.1460 & 0.6986 & 0.2567 \\
\hline 62 & 0.4191 & 0.3036 & 0.3987 & 0.2683 & 0.1511 & 0.3155 & 13.3064 \\
\hline 64 & 0.4037 & 0.4121 & 0.3412 & 0.5141 & 0.4034 & 0.2562 & 17.5858 \\
\hline 65 & 0.6595 & 0.5390 & 0.1599 & 0.4414 & 0.4525 & 0.1941 & 7.7303 \\
\hline 66 & 0.6725 & 0.5407 & 0.2537 & 0.5828 & 0.5036 & 0.2087 & 11.0421 \\
\hline 68 & 0.3085 & 0.2286 & 0.4282 & 0.1140 & 0.0685 & 0.3937 & 1.0344 \\
\hline Threshold & 0.0770 & & & & & & \\
\hline & $\begin{array}{l}\text { to } 34 \\
\text { to } 68\end{array}$ & son gra & & & & & \\
\hline
\end{tabular}


Table 13 Parameters of the curvelet-based RBF SVM Classifier C for prostate cancer Gleason grade 3 and grade 4

(34 P3 and 34 P4, average features from 9 patches of an image).

\begin{tabular}{|c|c|c|c|c|c|c|c|c|c|}
\hline Index & \multicolumn{8}{|c|}{ 8-feature Support Vectors } & Weights \\
\hline 3 & 0.2619 & 0.0616 & 0.2779 & 0.5755 & 0.5681 & 0.7984 & 0.5716 & 0.7401 & -23.2183 \\
\hline 4 & 0.4478 & 0.0232 & 0.1228 & 0.2799 & 0.2732 & 0.4679 & 0.3582 & 0.5595 & -0.7627 \\
\hline 5 & 0.5601 & 0.1125 & 0.2401 & 0.4952 & 0.4884 & 0.6840 & 0.7132 & 0.8336 & -20.4858 \\
\hline 6 & 0.4846 & 0.1601 & 0.2925 & 0.6286 & 0.4015 & 0.6878 & 0.6843 & 0.7934 & -25.4163 \\
\hline 7 & 0.6401 & 0.0568 & 0.2341 & 0.2636 & 0.1904 & 0.0581 & 0.1114 & 0.0000 & -0.5213 \\
\hline 10 & 0.7577 & 0.4219 & 0.2078 & 0.4408 & 0.1624 & 0.3405 & 0.0436 & 0.2144 & -1.2973 \\
\hline 11 & 0.7153 & 0.2151 & 0.5007 & 0.8935 & 0.7018 & 0.8835 & 0.9038 & 0.9586 & -4.2042 \\
\hline 12 & 0.0590 & 0.0175 & 0.2364 & 0.2791 & 0.2323 & 0.3422 & 0.3121 & 0.6092 & -5.0307 \\
\hline 14 & 0.7483 & 0.0507 & 0.0532 & 0.0000 & 0.0003 & 0.0000 & 0.0470 & 0.0442 & -1.0216 \\
\hline 15 & 0.2390 & 0.0167 & 0.1161 & 0.3423 & 0.3617 & 0.6469 & 0.5254 & 0.7953 & -14.4367 \\
\hline 16 & 0.2563 & 0.0223 & 0.1140 & 0.2947 & 0.0607 & 0.3387 & 0.1880 & 0.5241 & -4.6513 \\
\hline 17 & 0.1683 & 0.0128 & 0.1697 & 0.4568 & 0.3238 & 0.6210 & 0.4654 & 0.7501 & -13.5515 \\
\hline 20 & 0.4935 & 0.0817 & 0.0292 & 0.0444 & 0.0074 & 0.1147 & 0.2269 & 0.3442 & -0.0277 \\
\hline 21 & 0.5612 & 0.1281 & 0.2443 & 0.4965 & 0.4862 & 0.6831 & 0.7255 & 0.8338 & -9.0106 \\
\hline 22 & 0.6290 & 0.1788 & 0.1776 & 0.4939 & 0.2971 & 0.6331 & 0.5101 & 0.7310 & -6.9644 \\
\hline 24 & 0.4309 & 0.1009 & 0.2066 & 0.4205 & 0.3976 & 0.5333 & 0.3660 & 0.5049 & -9.8285 \\
\hline 25 & 0.3860 & 0.3287 & 0.2957 & 0.6747 & 0.3220 & 0.6364 & 0.2552 & 0.5428 & -10.1208 \\
\hline 26 & 0.4587 & 0.3746 & 0.2032 & 0.6028 & 0.2262 & 0.5569 & 0.1065 & 0.3858 & -3.8714 \\
\hline 29 & 0.5679 & 0.1101 & 0.0953 & 0.3440 & 0.3255 & 0.5772 & 0.4279 & 0.5666 & -0.2390 \\
\hline 32 & 0.8735 & 0.1339 & 0.0862 & 0.1596 & 0.2076 & 0.3213 & 0.3431 & 0.3740 & -0.3585 \\
\hline 33 & 0.5790 & 0.0635 & 0.1516 & 0.4050 & 0.2023 & 0.4595 & 0.4445 & 0.6431 & -4.7601 \\
\hline 34 & 0.5128 & 0.0776 & 0.1475 & 0.3586 & 0.0358 & 0.2536 & 0.1047 & 0.3412 & -0.7329 \\
\hline 35 & 0.8531 & 0.5826 & 0.3925 & 0.8439 & 0.8414 & 1.0000 & 1.0000 & 1.0000 & 0.9379 \\
\hline 36 & 1.0000 & 1.0000 & 0.3766 & 0.7887 & 0.7209 & 0.8982 & 0.8644 & 0.8455 & 0.1376 \\
\hline 37 & 0.5096 & 0.2327 & 0.2131 & 0.5417 & 0.3062 & 0.5888 & 0.2188 & 0.4801 & 11.2431 \\
\hline 38 & 0.4163 & 0.2032 & 0.2135 & 0.5238 & 0.3890 & 0.7017 & 0.5535 & 0.7509 & 17.7379 \\
\hline 40 & 0.2815 & 0.0864 & 0.3561 & 0.6571 & 0.5044 & 0.7165 & 0.6570 & 0.7786 & 13.4621 \\
\hline 41 & 0.3933 & 0.0413 & 0.1751 & 0.4436 & 0.5210 & 0.7418 & 0.7445 & 0.8512 & 11.6998 \\
\hline 42 & 0.3984 & 0.0428 & 0.1563 & 0.3845 & 0.2616 & 0.5587 & 0.3835 & 0.7185 & 9.2356 \\
\hline 43 & 0.6850 & 0.4007 & 0.2630 & 0.6507 & 0.3547 & 0.6501 & 0.6845 & 0.7914 & 6.1825 \\
\hline 44 & 0.9496 & 0.4352 & 0.5826 & 0.8488 & 0.8147 & 0.8262 & 0.9901 & 0.7257 & 1.0741 \\
\hline 48 & 0.6481 & 0.0443 & 0.4885 & 0.4993 & 0.5444 & 0.4494 & 0.6377 & 0.4934 & 4.2397 \\
\hline 49 & 0.8220 & 0.2225 & 0.4313 & 0.6917 & 0.5043 & 0.6791 & 0.3970 & 0.5251 & 0.4957 \\
\hline 50 & 0.9634 & 0.1967 & 0.2646 & 0.4894 & 0.6177 & 0.6916 & 0.6827 & 0.6167 & 0.9640 \\
\hline 53 & 0.0000 & 0.0040 & 0.0677 & 0.2477 & 0.3163 & 0.5872 & 0.4043 & 0.7243 & 7.7023 \\
\hline 56 & 0.3700 & 0.0683 & 0.0882 & 0.3570 & 0.2997 & 0.6442 & 0.4922 & 0.8145 & 6.9003 \\
\hline 57 & 0.5626 & 0.1110 & 1.0000 & 1.0000 & 1.0000 & 0.7725 & 0.4467 & 0.4369 & 0.4970 \\
\hline 59 & 0.2343 & 0.0768 & 0.1695 & 0.4912 & 0.4776 & 0.7949 & 0.5553 & 0.8430 & 7.1543 \\
\hline 61 & 0.1582 & 0.0855 & 0.0866 & 0.1870 & 0.1208 & 0.3164 & 0.4306 & 0.6409 & 3.7626 \\
\hline 62 & 0.4757 & 0.1680 & 0.1838 & 0.4532 & 0.2159 & 0.4647 & 0.4253 & 0.6152 & 6.5913 \\
\hline 63 & 0.6602 & 0.1170 & 0.2669 & 0.5798 & 0.4869 & 0.7285 & 0.7393 & 0.9084 & 17.2108 \\
\hline 64 & 0.4379 & 0.1020 & 0.3024 & 0.5726 & 0.5192 & 0.7295 & 0.6593 & 0.8263 & 18.5491 \\
\hline 65 & 0.1578 & 0.1075 & 0.2575 & 0.5070 & 0.3707 & 0.6062 & 0.3581 & 0.6418 & 11.3687 \\
\hline 67 & 0.1859 & 0.2270 & 0.1876 & 0.4214 & 0.0701 & 0.3556 & 0.0570 & 0.4646 & 0.5990 \\
\hline 68 & 0.2333 & 0.2175 & 0.1621 & 0.4326 & 0.0000 & 0.2868 & 0.0000 & 0.3598 & 2.7659 \\
\hline Threshold & 0.4880 & & & & & & & & \\
\hline
\end{tabular}

Pattern index 1 to 34 : Gleason grade 3

35 to 68 : Gleason grade 4 


\section{BIBLIOGRAPHY}

1. I. Daubechies, "Orthonormal bases of compactly supported wavelets," Communications on pure and applied mathematics, 41, 1988, Wiley Online Library, pp. 909-996.

2. S. G. Mallat, "A theory for multiresolution signal decomposition: The wavelet representation," Pattern Analysis and Machine Intelligence, IEEE Transactions on, 11, 1989, IEEE, pp. 674-693.

3. E. J. Candes, Ridgelets: theory and applications, Dept. of Statistics, Stanford University, 1998.

4. D. L. Donoho, "Orthonormal ridgelets and linear singularities," SIAM Journal on Mathematical Analysis, 31, 2000, Citeseer, pp. 1062-1099.

5. S. R. Deans, The Radon transform and some of its applications, Wiley, 1983.

6. J. S. Geronimo, D. P. Hardin, and P. R. Massopust, "Fractal functions and wavelet expansions based on several scaling functions," Journal of Approximation Theory, 78, 1994, Elsevier, pp. 373-401.

7. F. Keinert, Wavelets and Multiwavelets (Studies in Advanced Mathematics), Chapman and Hall/CRC, 2003.

8. I. W. Selesnick, "Interpolating multiwavelet bases and the sampling theorem," Signal Processing, IEEE Transactions on, 47, 1999, IEEE, pp. 1615-1621.

9. P. A. Toft, and J. A. Sørensen, The Radon transform-theory and implementation, Technical University of Denmark, Department of Informatics and Mathematical Modeling, 1996.

10. G. Y. Chen and B. Kegl, "Image Denoising with Complex Ridgelets," Pattern Recognition, vol. 40, 2007, pp. 578-585.

11. J. Ma, X. Jiang, and P. Scott, "Comple Ridgelets for Shift Invariant Characterization of Surface Topography with Line Singularities," Physics Letters A, vol. 344, 2005, pp. 423431.

12. E. J. Candes, D. L. Donoho, and Stanford University Department of Statistics., Curvelets: A surprisingly effective nonadaptive representation for objects with edges, Citeseer, 2000. 
13. E. J. Candès, and F. Guo, "New multiscale transforms, minimum total variation synthesis: Applications to edge-preserving image reconstruction," Signal Processing, 82, 2002, Elsevier, pp. 1519-1543.

14. G. Strang, and V. Strela, "Short wavelets and matrix dilation equations," Signal Processing, IEEE Transactions on, 43, 1995, IEEE, pp. 108-115.

15. X. G. Xia, J. S. Geronimo, D. P. Hardin, and B. W. Suter, "Design of prefilters for discrete multiwavelet transforms," Signal Processing, IEEE Transactions on, 44, 1996, IEEE, pp. 25-35.

16. J. Lebrun, and M. Vetterli, "Balanced multiwavelets theory and design," Signal Processing, IEEE Transactions on, 46, 1998, IEEE, pp. 1119-1125.

17. X. G. Xia, and Z. Zhang, "On sampling theorem, wavelets, and wavelet transforms," Signal Processing, IEEE Transactions on, 41, 1993, IEEE, pp. 3524-3535.

18. G. G. Walter, Wavelets and other orthogonal systems with applications, CRC press, 1994.

19. N. Kingsbury, "Complex wavelets for shift invariant analysis and filtering of signals," Applied and Computational Harmonic Analysis, 10, 2001, Elsevier, pp. 234-253.

20. N. Kingsbury, "The dual-tree complex wavelet transform: a new technique for shift invariance and directional filters," 1998.

21. I. W. Selesnick, "Hilbert transform pairs of wavelet bases," Signal Processing Letters, IEEE, 8, 2001, IEEE, pp. 170-173.

22. N. Kingsbury, "Image processing with complex wavelets," Philosophical Transactions of the Royal Society of London. Series A: Mathematical, Physical and Engineering Sciences, 357, 1999, The Royal Society, pp. 2543.

23. R. Yu, and H. Ozkaramanli, "Hilbert transform pairs of biorthogonal wavelet bases," Signal Processing, IEEE Transactions on, 54, 2006, IEEE, pp. 2119-2125.

24. X. Zhang, and D. F. Ge, "Hilbert Transform Pairs of Orthonormal Symmetric Wavelet Bases Using Allpass Filters," 1, 2007, IEEE, pp. I-425-I-428.

25. H. Shi, B. Hu, and J. Q. Zhang, "A novel scheme for the design of approximate Hilbert transform pairs of orthonormal wavelet bases," Signal Processing, IEEE Transactions on, 56, 2008, IEEE, pp. 2289-2297.

26. D. W. Wang and X. Zhang "Design of Hilbert transform pairs of orthonormal wavelet bases using IIR filters," Communications and Information Technologies (ISCIT), 2010 International Symposium on, 2010, IEEE, pp. 554-559.

27. I. W. Selesnick, R. G. Baraniuk, and N. C. Kingsbury, "The dual-tree complex wavelet transform," Signal Processing Magazine, IEEE, 22, 2005, IEEE, pp. 123-151. 
28. W. Y. Ma, and B. S. Manjunath, "Texture features and learning similarity," Computer Vision and Pattern Recognition, 1996 IEEE Computer Society Conference on, 1996, IEEE, pp. 425-430.

29. B. S. Manjunath, and W. Y. Ma, "Texture features for browsing and retrieval of image data," Pattern Analysis and Machine Intelligence, IEEE Transactions on, 18, 1996, IEEE, pp. 837-842.

30. V. Authors/Contributors, Convergent Technologies For The Asia-Pacific Region (TENCON - 2003, Four Volume Set), 2, IEEE, 2003.

31. M. Kokare, P. K. Biswas, and B. N. Chatterji, "Texture image retrieval using new rotated complex wavelet filters," Systems, Man, and Cybernetics, Part B: Cybernetics, IEEE Transactions on, 35, 2005, IEEE, pp. 1168-1178.

32. A. P. N. Vo, T. T. Nguyen, and S. Oraintara, "Image Denoising Using Shiftable Directional Pyramid and Scale Mixtures of Complex Gaussians," 2007 IEEE International Symposium on Circuits and Systems, IEEE, 2007, pp. 4000-4003.

33. S. Arivazhagan, L. Ganesan, and T. G. Subash Kumar, "Texture classification using ridgelet transform," Pattern recognition letters, 27, 2006, Elsevier, pp. 1875-1883.

34. P. Brodatz, Textures: a photographic album for artists and designers, Dover Publications, 1999.

35. J. S. Ryu, J. H. Oh, J. G. Kim, T. M. Koo, and K. H. Park, Tencon 2004: 2004 IEEE Region 10 Conference: Proceedings: Analog and Digital Techniques in Electrical Engineering: 21-24 November, Institute of Electrical \& Electronics Enginee, 2004.

36. A. B. Watson, "The spatial standard observer: a human vision model for display inspection," 37(1), 2006, pp. 1312-1315.

37. V. Vapnik, The Nature of Statistical Learning Theory (Information Science and Statistics), Springer, 1999.

38. K. M. Rajpoot, and N. M. Rajpoot, "Wavelets and support vector machines for texture classification," 2004, IEEE, pp. 328-333.

39. Y. J. Lee, and O. L. Mangasarian, "SSVM: A smooth support vector machine for classification," Computational optimization and Applications, 20, 2001, Springer, pp. 522.

40. Y. Zhang, and J. Zhang, Proceedings of 2005 International Conference on Neural Networks and Brain: Oct. 13-15, 2005, Beijing, China, 1, IEEE Computer Society Press, 2005.

41. Y. C. Song, D. H. Choi, and K. H. Park, "Morphological blob-mura defect detection method for TFT-LCD panel inspection," 2004, Springer, pp. 862-868. 
42. M. Lanyi, "Diagnosis and differential diagnosis of breast calcifications," 1987, SpringerVerlag New York Inc., New York, NY.

43. M. T. Freedman, D. E. S. Artz, H. Jafroudi, S. C. B. Lo, R. A. Zuurbier, R. Katial, W. S. Hayes, C. Y. Wu, J. S. Lin, and R. M. Steinman, "Digital mammography: tradeoffs between 50-and 100-micron pixel size," 2432, 1995, pp. 114.

44. E. J. Candes, L. Demanet, D. L. Donoho, and L. Ying, "Curvelab toolbox, version 2.0," 2005.

45. D. P. Chakraborty, "Recent advances in observer performance methodology: jackknife freeresponse ROC (JAFROC)," Radiation protection dosimetry, 114, 2005, NTP, pp. 26-31.

46. D. D. Dorfman, K. S. Berbaum, and C. E. Metz, "Receiver operating characteristic rating analysis: generalization to the population of readers and patients with the jackknife method," Investigative Radiology, 27, 1992, pp. 723-731.

47. X. Pan, and C. E. Metz, "The" proper" binormal model: parametric receiver operating characteristic curve estimation with degenerate data," Academic radiology, 4, 1997, pp. 380-389.

48. A. Jemal, R. Siegal, Y. Hao, J. Xu, and M. J. Thun, "Cancer Statistics 2010," CA Cancer J Clin 2010; 60, pp. 277-300.

49. J. I. Epstein, "An Update of the Gleason Grading System," J Urology, vol. 183, 2010, pp. 433-440.

50. D. F. Gleason, and G. T. Mellinger, "the Veterans Administration Cooperative Urological Research Group: Prediction of prognosis for prostatic adenocarcinoma by combined histological grading and clinical staging," J Urol, 111, 1974, pp. 58-64.

51. R. W. Veltr, A. W. Partin, and M. C. Miller, "Quantitative Nuclear Grade. Clinical Applications of the Quantitative Measurement of Nuclear Structure using Image Analysis," Cancer Chemoprevention, vol. 2: Edited by: G. J. Kelloff, E. T. Hawk, and C. C. Sigman (C) Humana Press Inc., Totowa, NJ, In Chapter 6, 2004, pp. 97-108.

52. R. W. Veltri, C. Marlow, M. A. Khan, M. C. Miller, J. I. Epstein, and A. W. Partin, "Significant Variations in Nuclear Structure Occur Between and Within Gleason Grading Patterns 3, 4, and 5 Determined by Digital Image Analysis," The Prostate, vol. 67, no. 11, May 2007, pp. 1202-1210.

53. K. Jafari-Khouzani, and H. Soltanian-Zadeh, "Multiwavelet grading of pathological images of prostate," Biomedical Engineering, IEEE Transactions on, 50, 2003, IEEE, pp. 697704.

54. M. Gao, P. Bridgman, and S. Kumar, "Computer aided prostate cancer diagnosis using image enhancement and JPEG2000," 5203, 2003, pp. 323-334. 
55. A. Zaim, T. Yi, and R. Keck, "Feature-based classification of prostate ultrasound images using multiwavelet and kernel support vector machines," 2007, IEEE, pp. 278-281.

56. A. Tabesh, M. Teverovskiy, H. Y. Pang, V. P. Kumar, D. Verbel, A. Kotsianti, and O. Saidi, "Multifeature prostate cancer diagnosis and Gleason grading of histological images," Medical Imaging, IEEE Transactions on, 26, 2007, IEEE, pp. 1366-1378.

57. S. Doyle, M. Hwang, K. Shah, A. Madabhushi, M. Feldman, and J. Tomaszeweski, "Automated grading of prostate cancer using architectural and textural image features," 2007, IEEE, pp. 1284-1287.

58. R. Farjam, H. Soltanian-Zadeh, K. Jafari-Khouzani, and R. A. Zoroofi, "An image analysis approach for automatic malignancy determination of prostate pathological images," Cytometry Part B: Clinical Cytometry, 72, 2007, Wiley Online Library, pp. 227-240.

59. S. Naik, S. Doyle, S. Agner, A. Madabhushi, M. Feldman, and J. Tomaszewski, "Automated gland and nuclei segmentation for grading of prostate and breast cancer histopathology," 2008, IEEE, pp. 284-287.

60. Y. Ou, D. Shen, J. Zeng, L. Sun, J. Moul, and C. Davatzikos, "Sampling the spatial patterns of cancer: Optimized biopsy procedures for estimating prostate cancer volume and Gleason Score," Medical image analysis, 13, 2009, Elsevier, pp. 609-620.

61. P. W. Huang, and C. H. Lee, "Automatic classification for pathological prostate images based on fractal analysis," Medical Imaging, IEEE Transactions on, 28, 2009, IEEE, pp. 1037-1050.

62. S. K. Tai, Y. C. Wu, C. Y. Li, Y. J. Jan, and S. C. Lin, "Computer-assisted Detection and Grading of Prostatic Cancer in Biopsy Image," Proceedings of the International MultiConference of Engineers and Computer Scientists, 1, 2010.

63. P. Khurd, C. Bahlmann, P. Maday, A. Kamen, S. Gibbs-Strauss, E. M. Genega, and J. V. Frangioni, "Computer-aided Gleason grading of prostate cancer histopathological images using texton forests," 2010, IEEE, pp. 636-639.

64. K. Nguyen, A. K. Jain, and R. L. Allen, “Automated Gland Segmentation and Classification for Gleason Grading of Prostate Tissue Images," 2010 International Conference on Pattern Recognition, 2010, IEEE, pp. 1497-1500.

65. K. A. Iczkowski, and M. S. Lucia, "Current Perspectives on Gleason Grading of Prostate Cancer," Current Urology Reports, Springer, pp. 1-7.

66. S. Canu, Y. Grandvalet, V. Guigue, and A. Rakotomamonjy, "SVM and kernel methods matlab toolbox," Perception Systmes et Information, INSA de Rouen, Rouen, France, 2, 2005. 